\title{
State Selective Dynamics of Molecules, Clusters, and Nanostructures
}

Final Progress Report

May 1, 1993-Feb. 29, 2005

\author{
J. W. Keto \\ Physics Department \\ The University of Texas at Austin \\ Austin, Tx. 78712
}

June 2005

Prepared for

The U.S. Department of Energy

under Grant No. DE-FG03-93-ER14348 


\section{Table of Contents}

$\begin{array}{lll}1.0 & \text { Introduction } & 4\end{array}$

2.0 Program Milestones 5

3.0. Two-photon spectra of excitons in solid xenon $\quad 7$

$\begin{array}{lll}\text { 3.1. Introduction } & 7\end{array}$

3.2. Results for solid xenon 8

4.0. Laser spectroscopy of Xenon excimers in a supersonic jet 10

$\begin{array}{lll}\text { 4.1. Apparatus } & 10\end{array}$

$\begin{array}{lll}4.2 & \text { Results } & 11\end{array}$

$\begin{array}{lll}4.3 & \text { Spectra of small clusters } & 12\end{array}$

5.0. State-to-state energy transfer from $\mathrm{Xe}^{*} 5 \mathrm{p}^{5} \mathrm{nd}$ in collisions with rare gas atoms 16

$\begin{array}{ll}\text { 5.1. Introduction } & 16\end{array}$

5.2. State-to-state collision studies of $\mathrm{Xe}^{*} 5 \mathrm{p}^{5} 6 \mathrm{~d}$ multiplets

$\begin{array}{ll}\text { using a two-color experiment } & 19\end{array}$

5.3. Experimental studies of the $5 p^{5} 6 \mathrm{~d}$ multiplets 22

6.0. Laser assisted half-collisions in reaction of $\mathrm{Xe}$ and $\mathrm{Cl}_{2}$

6.1. Experimental approach 25

6.2 Simulation of Bound-Free Spectra 28

6.3. Surprisal Analysis 29

6.4 A Simple Repulsive Release Model 30 
7.0 Coherent Raman Spectroscopy of Ions and Radicals in Discharges

7.1 Progress for obtaining OHD-RIKES of discharges

7.1.1 Improvements in $\mathrm{S} / \mathrm{N}$ with RIKES

7.1.2 Heterodyne experiments

7.1.3 Improvements in the discharge circuitry, electrode geometry, and synchronization electronics.

7.2 Experiments in a low pressure discharge

7.2.1 Attempts to observe the $\mathrm{N}_{3}$ radical.

7.3.2 The $\mathrm{O}_{2}$ hotband and $\mathrm{O}_{2}{ }^{1} \Delta$

7.3.3 $\mathrm{CO}_{2}$ in a discharge: quantum interference in $\mathrm{V}-\mathrm{T}$ relaxations

8.1 Single-particle spectra: Microluminescence and NSOM

8.2 Single particle spectroscopy of nanoparticles

8.3 Nanoparticles collected in a surfactant

8.4 Semiconductor nanoparticles in surfactants

8.4.1. Silicon nanoparticles captured in ethylene glycol 56

8.5 Impurity Doped Nanoparticles

9.0 Publications

$10.0 \quad$ Presentations

$11.0 \quad$ Personnel 


\subsection{Introduction}

The objective of this grant has been the study of state-to-state, electronic energy transfer reactions relevant to the excited state chemistry observed in a variety of environments- mixed gases, supersonic jets, discharges, and in condensed matter. The reactant states are excited selectively by one or two-photon transitions driven by a tunable u.v. lasers. Product channels are observed by their fluorescence, or by laser induced fluorescence using a second color laser, or by Raman transitions observed by coherent two-color four wave mixing. An earlier grant explored excitation transfer reactions in rare gases and rare gaseds doped with chorides. These reactions were important to both excimer lasers and the nuclear pumped rare gas laser. More recent experiments have studied laser assisted reactive scattering, energy transfer in condensed rare gases and in clusters formed in supersonic jets. Toward the end of the grant we developed a technique for the manufacture of nanoparticles: Laser Abalation of Microparticles (LAM) in flowing aersols. We have use state selective laser spectroscopy to study clusters and nanoparticles produced by this method.

\subsection{Program Milestones}

Although one-photon absorption was studied previously in condensed xenon, 1,2,3 we measured the first two-photon excitation spectra at the band edge of liquid and solid xenon in which direct excitation of the even-parity exciton levels is observed. These measurements were the first ever observation of the even parity excitons in any of the rare gas solids. Several groups had attempted these experiments unsuccessfully, but were unable to grow the large single crystals of xenon required. Justification for measurements of the excitonic structure of condensed rare gases lies mainly in the close correspondence between the fluorescence of the trapped exciton in the liquid and the excimer in the gas. Liquid rare gases have been studied as possible excimer lasers systems, both as pure systems ${ }^{4}$ and doped with halogens. ${ }^{5}$ These experiments are summarized briefly in Sec. 3; details were published in Publications 4, 7 and 9 listed in Sec. $10 .$.

We completed a study of the spectroscopy of bound, gerade xenon excimers produced by twophoton excitation of dimers formed in supersonic jets. The molecules studied were relevant to knowledge of the interaction potentials for collision studies. We also obtained two-photon spectra of xenon atoms and dimers solvated in both He and Ar clusters. We observed magic numbers in the structure of these solvated clusters. For He clusters one or two rings of six He atoms surrounding the Xe dimers. The number is just consistent with the dimer separation of He atoms in the formation of the ring about the dimer. For argon solvation, magic numbers were consistent with rings of five Ar atoms about the dimer. We observed banded fluorescence spectra of Xe excimers solvated in He clusters. This fluorescence has never been observe for the bare Xe excimer and likely comes from the prevented predissociation of the excimer by the presence of the He clusters. These experiments are review briefly in Sec 4 and more details are contained in Publications 11, 14, and 29 in Sec. 10.

1G.Baldini, Phys. Rev. 128, 1562 (1962)

2D. Beaglehole, Phys. Rev. Lett. 15, 553 (1965)

3E. Roick, R. Gaethke, G. Zimmerer, Solid State Communic. 47, 333 (1983)

${ }^{4} \mathrm{~N}$. Schwentner, "Photodissociation of small molecules and generation of laser radiation in rare-gas crystals", J. Mole. Struct. 222, 151 (1990).

${ }^{5} \mathrm{~K}$. Okada and V. A. Apkarian, "Electronic relaxation of $\mathrm{Xe}_{2} \mathrm{Cl}$ in gaseou and supercritical fluid xenon", J. Chem. Phys. 94, 133 (1991). 
We completed a manuscript describing measurements of rates for state-to-state deactivation, rates for excitation transfer, and radiative lifetimes for $\mathrm{Xe}^{*}\left(6 \mathrm{p}, 6 \mathrm{p}^{\prime}, 7 \mathrm{p}\right)$ and $\mathrm{Kr} *(5 \mathrm{p})$ states in xenon and krypton buffer gases. These results are relevant to kinetic models rare-gas discharges used in excimer lasers, the nuclear pumped xenon laser, processing plasmas, and discharge lamps (particularly $\mathrm{Hg}$ and $\mathrm{Na}$ arc lamps using Xe buffer gases). We also report experiments to measure the state-to-state deactivation cross sections and radiative lifetimes for $\mathrm{Xe} *(5 \mathrm{~d}$ and $6 \mathrm{~d})$ in rare-gas butters using two-color laser experiments. These results are relevant to kinetic models of both excimer lasers and the infrared xenon laser. 6,7 Our progress on these experiments are described in Sec. 5 which were published in the thesis of Joe Holder, Publication 15.

We have completed successfully a study of two- photon laser assisted collisions in the reaction

$$
\mathrm{Xe}+\mathrm{Cl}_{2}+\mathrm{h} \omega \rightarrow\left(\mathrm{Xe}^{+*} \mathrm{Cl}_{2}^{-}\right) \rightarrow \mathrm{XeCl} *(\text { high } v)+\mathrm{Cl}
$$

These experiments, spectral modeling, and a model for the reaction dynamics for this process are described in detail in Sec. 6. This work was the thesis of Jim Kohel, Publication 12; which was also published in the J. of Chem. Phys. Ref.i27. This research was beautifully detailed and measured surprisal functions for energy disposal in both rotation and vibrational states of the $\mathrm{XeCl}^{*}$ product. All of the experimental measurements were in agreement with a numerical calculation using a model assuming a direct interaction with product repulsion (first suggested by Polanyi) on the $\mathrm{Xe}^{+}+\mathrm{Cl}_{2}^{-}$energy surface.

An important problem in high pressure discharges is the understanding of the reactions of ions and radicals. At pressures near one atmosphere, new ions in the form of clusters are formed and reactions of all forms of ions are complicated by the possibility of termolecular reactions. ${ }^{8,9}$ Studies of high pressures discharges have been prevented by a lack of diagnostic for the interesting species. At low pressures mass spectrometers are effective but at atmospheric pressures the sampling of cluster ions through a differentially pumped orifice produces a supersonic expansion causing the size of the cluster to grow. Spontaneous Raman spectroscopy and infrared adsorption are hampered by the high background luminosities. We improved the sensitivity of Coherent Raman spectroscopy sufficiently so that it can be used detect the spectra of ions and radicals in high pressure discharges. We succeeded in measuring the spectrum of the $\mathrm{O}_{2}{ }^{1} \Delta$ metastable state excited state in a moderate pressure positive column glow discharge. We also measured trace ( $1 \mathrm{ppm}$ ) of $\mathrm{CO}_{2}$ in air. We then built a high pressure transfersely excited discharge capable of producing large densities of cluster ions and radicals. We made systematice searches for $\mathrm{N}_{3}$ and $\mathrm{N}_{2}+$ in this high pressure discharge. Our experiments in this area are described in Sec. 7.

In the last couple of years of the DOE grant our interest shifted to the measurement of the surface and bulk electronic quantum states of nanocrystals produced by a unique method of synthesis--laser ablation of microspheres (LAM); surface defect states produced by this technique

6. Mieko Ohwa, Thomas J. Moratz, and Mark J. Kushner, "Excitation mechanisms of the electron-beampumped atomic xenon (5d-6p) laser in Ar/Xe Mixtures", J. Appl. Phys. 66, 5131(1989).

7. W. J. Alford, "State-to-state rate constants for quench9ing of xenon $6 p$ levels by rare gases", J. Chem. Phys. 96, 4330(1992).

8. Chien-Yu Kuo and J.W. Keto, "Dissociative Recombination of Electrons in Electron Beam Excited Argon and High Densities," J. Chem. Phys. 78, 1851-1860 (1983).

${ }^{9}$ D. R. Bates and W. Lowell Morgan, Phys. Rev. Lett. 64, 2258(1990). 
are expected to be different from those grown chemically; and the knowledge of their defect states are essential to their use as optical devices, and (3) study the dynamics of excitons in structures of nanoparticles. Using near-field scanning microscopy (NSOM) and microluminescence techniques, we worked to obtain the spectra of single nanocrystals and correlate their spectra with size. These experiments are described in Sec. 8.2.

In Sec. 8.3 we describe experiments studying the stabilization of silver nanoparticles in the surfactant nonanoic acid. When nanoparticles are grown colloidally the particle are in equilibrium at high temperatures, while with LAM the particles are impacted cold into the surfactant. We found that the conformation of the surfactant molecule is in a state lying. down on the surface of the nanoparticle. We found the molecule made a transition to a vertical state on the surface when solutions are annealed at temperatures from 70 t0 $100 \mathrm{C}$. We measured the reaction rate and confirmed the molecular orientation with surface enhance Raman spectroscopy.

In Sec. 8.4 we report studies of the fluorescence of semiconductor nanoparticles manufactured by LAM and collected in surfactants. We found that CdSe nanoparticles were fluorescence if collected dry on sapphire substrates. We then studied CdSe and Si nanoparticles in surfactants. In Sec. 8.5 we manufactured impurity doped nanoparticles. These preliminary results suggest the importance of LAM for the manufacture of nanoparticles for use in direct write of waveguides with gain in integrated optics. When such particles are collected in a surfactant and solution they will have important applications for a high-power, nanoslush laser. 


\subsection{Two-photon spectra of excitons in solid xenon}

\subsection{Introduction}

Although one-photon absorption has been studied previously in condensed xenon, $10,11,12$ we measured the first two-photon excitation spectra through the band edge of liquid and solid xenon in which direct excitation of the even-parity exciton levels is observed. These measurements were the first ever observation of the even parity excitons in any of the rare gas solids. Justification for measurements of the excitonic structure of condensed rare gases lies mainly in the close correspondence between the fluorescence of the trapped exciton in the liquid and the excimer in the gas. Liquid rare gases have been studied as possible excimer lasers systems, both as pure systems ${ }^{13}$ and doped with halogens. ${ }^{14}$

We previously reported our experimental results on liquid xenon. ${ }^{15}$ The Wannier model of excitons in a solid (or liquid) assumes the bound electron orbits the hole (ion) imbedded in a smooth dielectric. The dielectric screening reduces attraction of the charge and increases the size of the electron's orbit. The mass of the electron is increased and is described by the change in band energy with $\mathrm{k}$ in the vicinity of the transition point in the Brillouin zone. Because of the large electron radius, the energy of the exciton does not depend upon the structure of the hole, and the energy levels of the excitons are describable by a simple hydrogen model

$$
E_{n}=E_{g}-\frac{B}{n^{2}},
$$

where $E_{g}$ is the band gap energy, $B=\frac{\mu R}{\varepsilon^{2}}$ is the exciton binding energy, $R$ is the atomic Rydberg constant, $\varepsilon$ is the dielectric constant of the medium, and $\mu$ is the effective mass of the electron in the solid. As in hydrogen, we expect both even and odd parity excitons of given principle quantum number $\mathrm{n}$ to be degenerate.

The one-photon excitation scans of Beaglehole 22 and Subtil, et. al. ${ }^{16}$ in liquid xenon yield linewidths consistent with our experiment, but, while the $2 \mathrm{~s}$, odd-parity excitons observed in those experiments are shifted by approximately $0.03 \mathrm{eV}$ to the red $22,27,17$ relative to the $2 \mathrm{~s}$ exciton in the solid at $160 \mathrm{~K}$, the even-parity bands excited by two-photon absorption in the liquid are shifted approximately $0.085 \mathrm{eV}$ towards the blue. We presented a model estimating the shift between the $2 s$ and $2 p$ excitons due to central cell corrections which is consistent with the measured shift of $0.115 \mathrm{eV}$. As the electron approaches the hole within the central cell the dielectric screening

10G.Baldini, Phys. Rev. 128, 1562 (1962)

11D. Beaglehole, Phys. Rev. Lett. 15, 553 (1965)

12E. Roick, R. Gaethke, G. Zimmerer, Solid State Communic. 47, 333 (1983)

${ }^{13}$ N. Schwentner, "Photodissociation of small molecules and generation of laser radiation in rare-gas crystals", J. Mole. Struct. 222, 151 (1990).

${ }^{14} \mathrm{~K}$. Okada and V. A. Apkarian, "Electronic relaxation of $\mathrm{Xe}_{2} \mathrm{Cl}$ in gaseou and supercritical fluid xenon", $\mathrm{J}$. Chem. Phys. 94, 133 (1991).

${ }^{15}$ C. A. Whithead, H. Pournasr, and J. W. Keto, Phys. Rev. B 47, 4979 (1993).

16. L. Subtil, P. Laporte, R. Reininger, and V. Saile, Phys. Stat. Sol. (b) 143, 783, (1987).

${ }^{17}$ B. SonnTag, "Dielectric and Optical Properties", Rare Gas Solids Vol II, M.L. Klein and J. A. Venables, eds., Academic Press, London, NewYork, 1977, p. 1049. 
diminishes with $\varepsilon$ approaching one at $r=0$ and the effective mass $\mu$ approaching the free mass of the electron. There is an additional polarization charge due to the hopping of the exciton in the solid. These central cell corrections were described by Hermanson. ${ }^{18}$ in an attempt to describe the shift of the $n=1$ exciton from those of higher $n$.

At the time of these experiments we were unable to grow crystals of sufficient clarity for transmission of the laser through the $1 \mathrm{~cm}$ path. Homer Pournasr designed a new chamber so as to minimize differences in expansion with the crystal. By careful adjustment of the growth rate (growth and annealing required several hours) defect-free crystals of $1 \mathrm{~cm}$ dimension were obtained. These could be cooled very slowly to temperatures as low as $100 \mathrm{~K}$. Defects were formed, again by differential expansion, but these could be removed by annealing over a 10 hour period.

\subsection{Results for solid xenon}

We obtained excitation spectra of two-photon excited solid xenon in the energy range of $8.4 \mathrm{eV}$ to $11.1 \mathrm{eV}$. The spectra he obtained of these crystals are truly beautiful. An example spectra is observed in Fig. 3.1 The signal-to-noise is much superior to that obtained for odd parity excitons using synchrotrons. The $n=4$ exciton was observed for the first time, and because of the absence of the band edge, which is not two-photon allowed, he was able to observe unresolved transitions to several excitons with $n>4$.

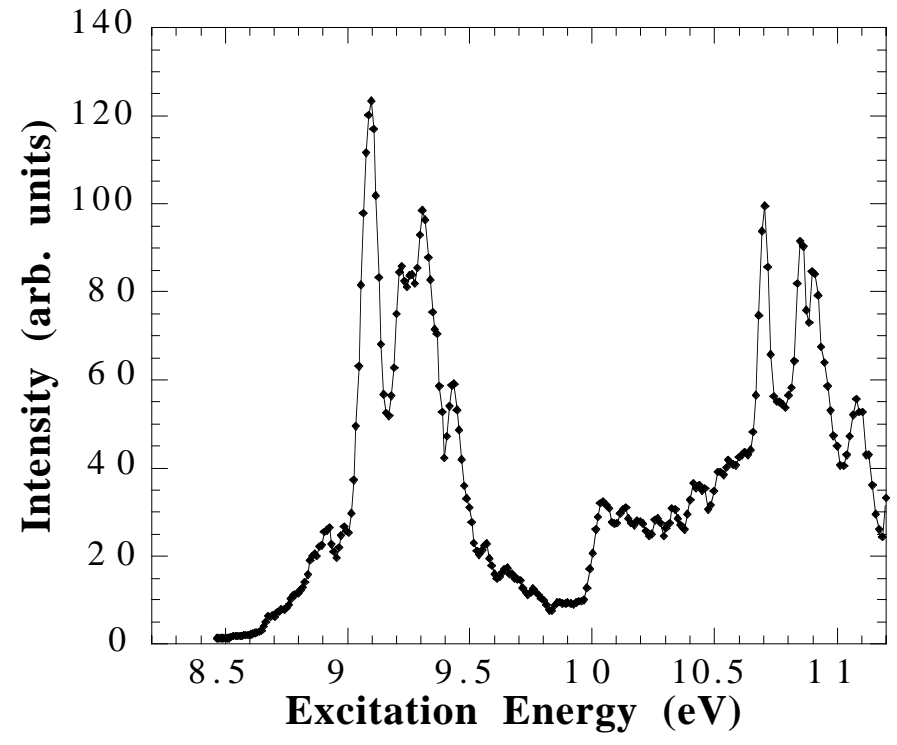

Fig. 3.1. Two-photon excitation spectra obtained in solid xenon at $158 \mathrm{~K}$.

We measured the linewidths and positions of the excitons as a function of temperature from 80 to $160 \mathrm{~K}$. The decrease in line width with increasing $\mathrm{n}$ and the line shapes are consistent with dynamical relaxation of the hole by collisions with phonons. Because of improved signal-to-noise in the new experiments, we identified weak two-photon transitions to both $n=1$ and n'=1 odd parity

18 J. Hermanson, Phys. Rev. 150, 660(1966). 
excitons in both the liquid and solid spectra. Forbidden two-photon transitions to odd parity excitons becomes weakly allowed because their point symmetry is broken by the crystal field.

Central cell corrections to the Wannier model in the solid failed to explain the shift in energy between the even and odd parity excitons. There was a near cancellation between the contributions to the energy from the polarization potential and the change in effective mass within the central zone. We suggested that better band-structure calculations for xenon might alter this cancellation and possibly explain the shift; however the current theory of excitons in the solid rare gases is incapable of explaining the reported experimental results.

We reported a second Rydberg series of excitons above the conduction band (seen at $10.7 \mathrm{eV}$ in Fig. 3.1). We tentatively assign this series to transitions at the $X$ point in the Brillouin zone. No other spectroscopic techniques have observed transitions in this energy range, or at the X point of the Brillouin zone. There is a systematic shift in the observed exciton energies from those expected from the current best band structure calculations reported in the literature. Homer Pournasr completed his Ph.D. thesis on this project, and the experiments are described in detail in publications 4 and 7 listed in the publications subsection. 


\subsection{Laser spectroscopy of Xenon excimers in a supersonic jet}

We measured the structure of excimers bound in potentials dissociating to the $\mathrm{Xe}^{*} 6 \mathrm{p}[1 / 2]_{0}$, $6 \mathrm{p}[3 / 2]_{2}$, and $6 \mathrm{p}[5 / 2]_{2}$ by exciting dimers formed in supersonic beams. A laser excitation spectra is shown in Fig. 4.1 Dehmer et al(19) first observed these resonances in the photoionization spectrum near $\mathrm{Xe}^{*}\left(5 \mathrm{p}^{5} 6 \mathrm{p}\right.$ and $\left.5 \mathrm{p}^{5} 5 \mathrm{~d}\right)$ which they interpreted as vibrational sequences for bound excimers dissociating to the nearby atomic levels. An assignment of the vibrational states was not possible because of the expected rapidly varying FrankCondon factors near possible curve crossings and because of the large equilibrium distance of the dimer. We proposed to assign the vibrational states by observing the fluorescence spectra of selectively excited vibrational levels. By measuring the fluorescence spectra of the $6 \mathrm{p}[1 / 2]_{0} 0 \mathrm{~g}^{+} \rightarrow 6 \mathrm{~s}[3 / 2]_{1}$ $1_{\mathrm{u}}$ transition, we hoped to be able to assign the sequence absolutely. In bound-bound spectra which are shifted in mean equilibrium atomic separation, $\mathrm{R}_{\mathrm{e}}$, the Frank-Condon factors are modulated by the upper state potential. By counting the nodes in the modulation, the upper state vibrational assignment can be made. Similar modulations can be observed in bound-free spectra.

\subsection{Apparatus}

The supersonic beam apparatus employs four differentially pumped chambers. The first houses a pulsed supersonic nozzle. A skimmer isolates this excimers measured by collecting only $\mathrm{Xe}_{2}{ }^{+}$ stagnation chamber from the second, laser photoions from the TOF spectra.

interaction chamber. The third chamber houses a quadrupole mass spectrometer (Extranuclear, Inc.) with a mass range of 400 and resolution of better than one amu. The vacuum system has been designed for ultra high vacuum in order to minimize ionization of the background gas when using u.v. lasers and resonance ionization detection. The laser is focused diagonally into the laser interaction chamber through a baffled window and is dumped into a high-quality Wood's horn to minimize scattered laser light. Experimental tests have measured less than 3 photons of scattered light per 1000 laser shots. A block diagram of the apparatus is shown in Fig. 4.2

${ }^{19}$ P. M. Dehmer, S. T. Pratt, and J. L. Dehmer, J. Chem. Phys. 85, 13 (1986). 
The ion detector is a deflection, time-of-flight mass spectrometer for use with ions produced by a twophoton, resonance excited, onephoton photoionization process $(2+1$ REMPI). This technique is very sensitive and enables the measurement of excitation spectra of excited molecules and clusters unstable for fluorescence. Since the detection is mass selective, excitation bands can be assigned to specific clusters.

\subsection{Results}

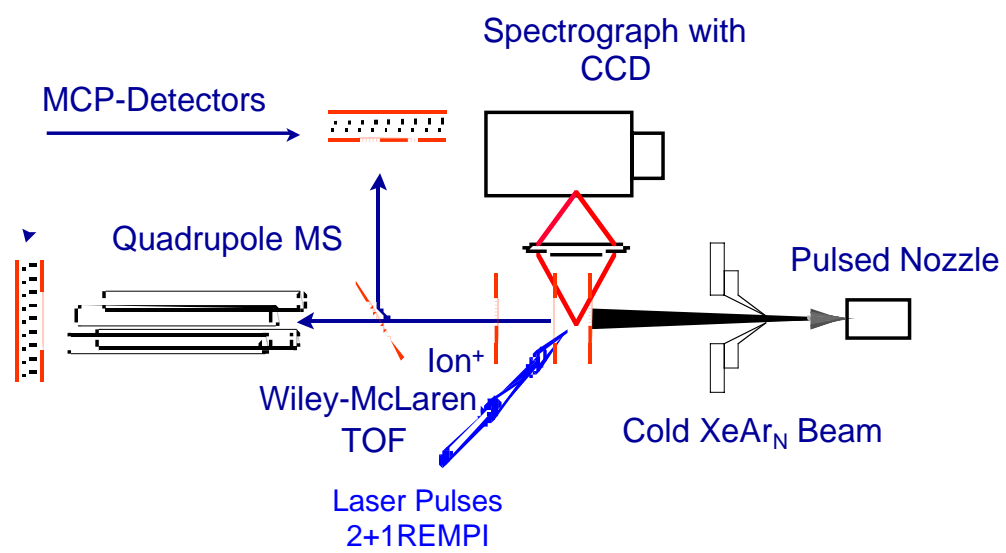

Fluorescence light was collected from $\pi / 2$ steradians, f matched to a $1 / 4$ meter spectrograph with an $\mathrm{f} 4$ aperature, and detected using a liquid nitrogen cooled CCD array detector (Princeton LN TK512B) with $60 \%$ QE at $828 \mathrm{~nm}$. The measured atomic fluorescence from $\mathrm{Xe}^{*} 6 \mathrm{p}[1 / 2]_{0}$ was 80 photo-e-/laser shot. Scaling by the monomer/dimer ratio $(\sim 120 / 1$ for $8 \% \mathrm{Xe} / \mathrm{He}$ at a stagnation pressure of 1000 Torr) measured from TOF spectra, we expected an excimer fluorescence of 0.7 photons/laser shot. When tuned to a $\mathrm{Xe}_{2} * 0_{\mathrm{g}}{ }^{+}$ excimer resonance at $249.9656 \mathrm{~nm}$, we observed less than $2 \times 10^{-4}$ photo-e-/laser shot. The lack of fluorescence is explainable by predissociation. We have searched unsuccessfully with similar sensitivity for fluorescence from all possible dissociation products of energy lower than $\mathrm{Xe}^{*}$ $6 \mathrm{p}[1 / 2]_{0}$, except for states of $\mathrm{Xe}^{*} 6 \mathrm{~s}^{\prime}$ and $\mathrm{Xe}^{*} 6 \mathrm{~s}$, which are undetectable in the present experiments. We conclude these are the likely product states.

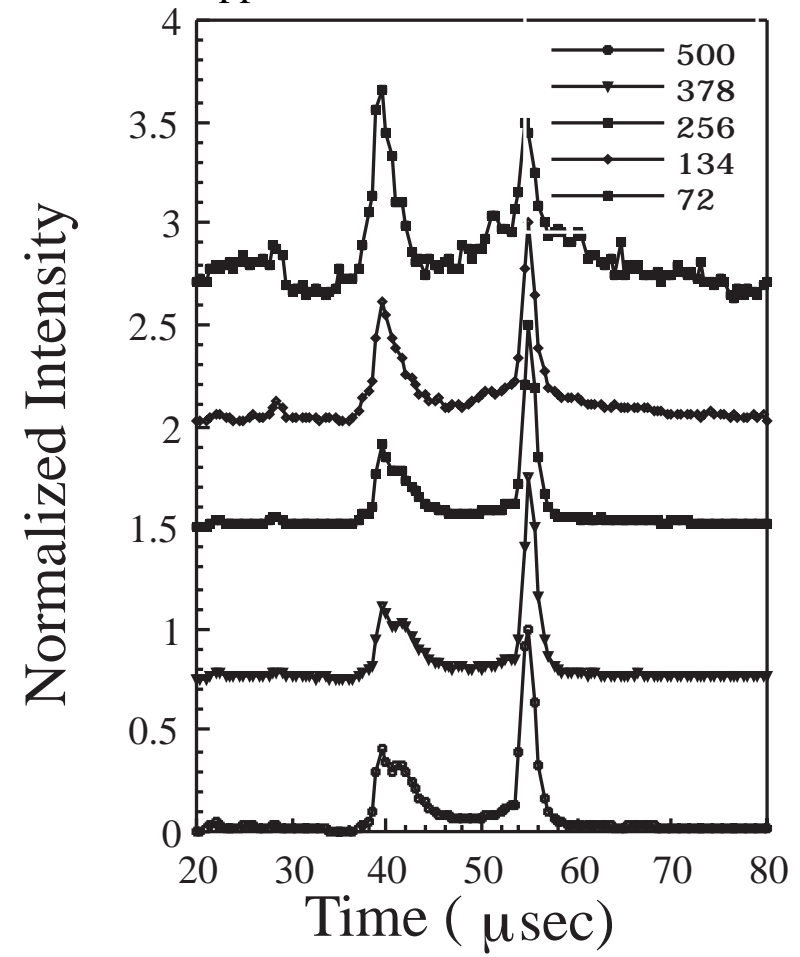

Predissociation was also confirmed by measuring the $\mathrm{Xe}_{2}+\rightarrow \mathrm{Xe}^{+}$fragmentation observed in the time-of-flight (TOF) spectrum, shown in Fig. 4.3.. At the highest laser powers, $\tau_{\mathrm{vib}}<\tau_{\text {photoion }}$ $\sim \tau_{\text {diss }}<\tau_{\text {rad }}$ for the excimer state, and little fragmentation was observed; as the laser power was

Fig. 4.3. TOF spectra while exciting a dimer resonance at 249.9656. Plotted is the normalized ion count/channel plotted as a function of delay time. The curves are organized from low to high power with a fixed offset of 0.75 . The peak at $40 \mu \mathrm{s}$ is $\mathrm{Xe}^{+}$; that at $55 \mu \mathrm{sec}$ is $\mathrm{Xe}_{2}{ }^{+}$.

decreased, predissociation begins to compete with photoionization and fragmentation to the $\mathrm{Xe}^{+}$ channel increased. We also observe that the monomer ion peak is considerably broader than the dimer ion peak. The structure of this peak has been studied as a function of the electric field in the ionization region between the back and first grids. As the fields decrease the dimer ion and the slowest monomer ion decrease in intensity. At a slight negative field only the fastest peak remains. We assign the slowest and fastest monomer peaks to $\mathrm{Xe}^{+}$ions formed by photoionization of the excited atoms resulting from predissociation of the excimer. In the center of mass motion of the jet the dissociated ions explode radially at all angles; but the ions exploding transverse to the beam do not reach the ion detector after the drift tube, as we estimate that ions with transverse velocities much above kT will not reach the detector. The central peak is formed by direct photoionization from the 
excimer to dissociative molecular ion potentials. We shall see these results are consistent with new fluorescence experiments. From these data and linewidths measured in spectra similar to Fig. 4.1 we estimate predissociation lifetimes on the order of $10^{-11} \mathrm{~s}$.

Shortly after these experiments were completed, Richmann, et. al. ${ }^{20}$ reported fluorescence while exciting the same vibronic state of $\mathrm{Xe}_{2}{ }^{*}$ at 249.9656 as described above. This fluorescence was obtained for $5 \% \mathrm{Xe} / \mathrm{He}$ at 4600 Torr stagnation pressure. They isolated the fluorescence to $765-830 \mathrm{~nm}$ in wavelength using cutoff filters. Under similar conditions, we have obtained the dispersed fluorescence spectra shown in Fig. 4.4. The TOF mass spectrum of photoions produced at the same wavelength and experimental conditions show satellites on the high mass side, suggesting shells of helium atoms surrounding the xenon dimer.

In Fig. 4.5, we plot the fluorescence intensity and the $\mathrm{Xe}_{2}{ }^{+}$photoionization intensity as a function of time after the nozzle is opened. Maximum fluorescence occurs at a time well after the dimer ion concentration has decreased. We observe sharp mass peaks early in time and at the maximum in $\mathrm{Xe}_{2}{ }^{+}$signal. These broaden on the high mass side later in the pulse as the fluorescence intensity

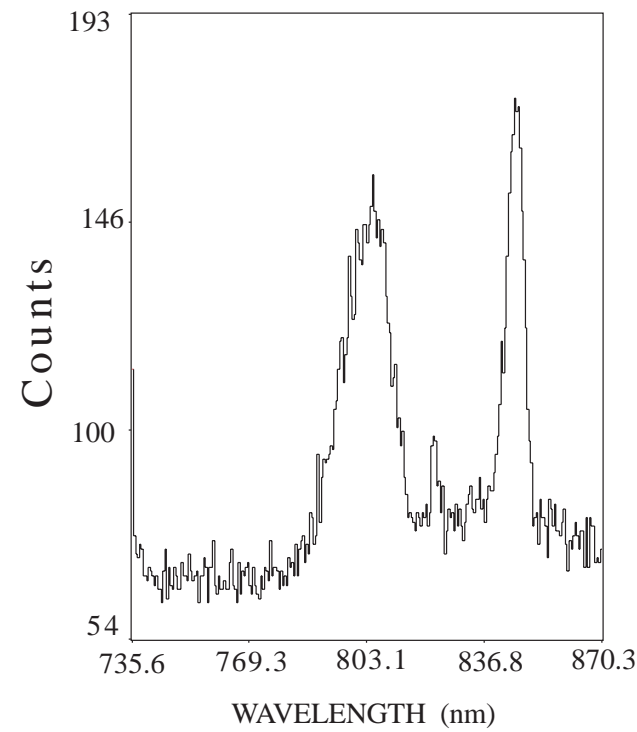

Fig. 4.4. Fluorescence spectra obtained when exciting a $\mathrm{O}_{\mathrm{g}}{ }^{+}$resonance near $\mathrm{Xe}^{*} 6 \mathrm{p}[1 / 2]_{0}$ for $5 \% \mathrm{Xe} / \mathrm{He}$ at a total stagnation pressure of 3000 Torr. increases. We propose that the fluorescence occurs for xenon dimers excited within a helium cluster. These clusters grow larger later in the nozzle pulse. This is consistent with experiments at higher xenon concentrations, where the mean size of xenon clusters increases with time for up to 3 $\mathrm{ms}$ after the nozzle opens. Within the helium cluster, collisions with helium atoms deactivate the predissociative $\mathrm{Xe}_{2} * 0_{\mathrm{g}}{ }^{+}$molecule to form a stable and fluorescent $\mathrm{Xe}_{2} * 0_{\mathrm{u}}{ }^{+}$. These experiments were published in Ref. .

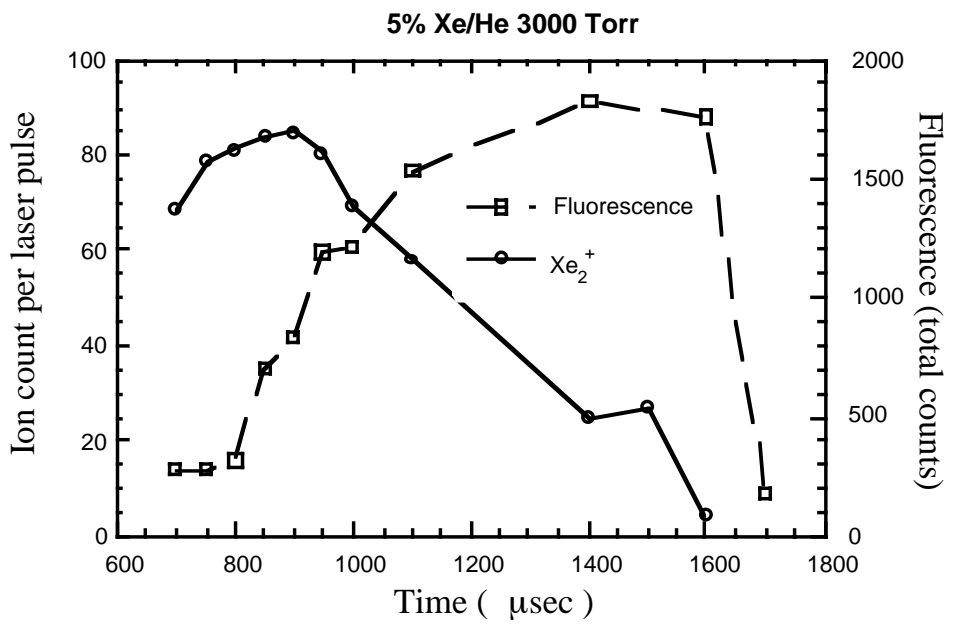

Fig. 4.5. $\mathrm{Xe}_{2}{ }^{+}$signal in the photoionization spectra plotted as a function of time after the supersonic nozzle is opened. Also shown the integrated fluorescence of the spectra shown in Fig. 4.4, plotted as a function of time.

\subsection{Spectroscopy of XeArn}

20 M. K. Richmann, T. O. Nelson, and D. W. Setser, Chem. Phys. Letters 210, 71(1993). 
Experiments were also conducted in xenon-argon mixtures. Photoionization and fluorescence spectra of $\mathrm{XeHe}_{\mathrm{n}}$ clusters were shown in Sec. 4.2. The analysis of the data suggests that the Xe dimer is embedded in a helium cluster at the time of excitation. After the excitation, the $\mathrm{Xe}_{2} *$ can be pushed out of the cluster (whereby interaction between the cluster and the excimer stabilizes the excimer against predissociation) and fluoresce undisturbed by the cluster, or it can fluoresce from inside the cluster, causing the observed broadening. By comparing $\mathrm{Xe}_{2}{ }^{*}$ fluorescence in argon and helium clusters, the correct case can be determined.

\subsubsection{Experimental}

Excitation spectra of $0.5 \%$ xenon in argon are shown in Fig. 4.6. They include the vicinities of the resonances $6 \mathrm{p}[1 / 2]_{0}\left(\right.$ or ${ }^{2} \mathrm{P}_{5}$ ) at $249.627 \mathrm{~nm}, 6 \mathrm{p}[3 / 2]_{2}\left(\right.$ or ${ }^{2} \mathrm{P}_{6}$ ) at $252.484 \mathrm{~nm}$ and $6 \mathrm{p}[5 / 2]_{2}$ (or ${ }^{2} \mathrm{P}_{9}$ ) at $256.015 \mathrm{~nm}$. New features appear in these spectra and are indicated by the arrows. The bars show the positions of the lines for the Xe-Ar dimer which Pratt et al. ${ }^{21}$ found in a continuous supersonic expansion of a 1:8 mixture of xenon and argon. The authors do not show spectral features between the bands of lines clustered around the resonances Xe6p[1/2 $]_{0}, \mathrm{Xe} 6 \mathrm{p}[3 / 2]_{2}$, and $\mathrm{Xe} 6 \mathrm{p}[5 / 2]_{2}$.
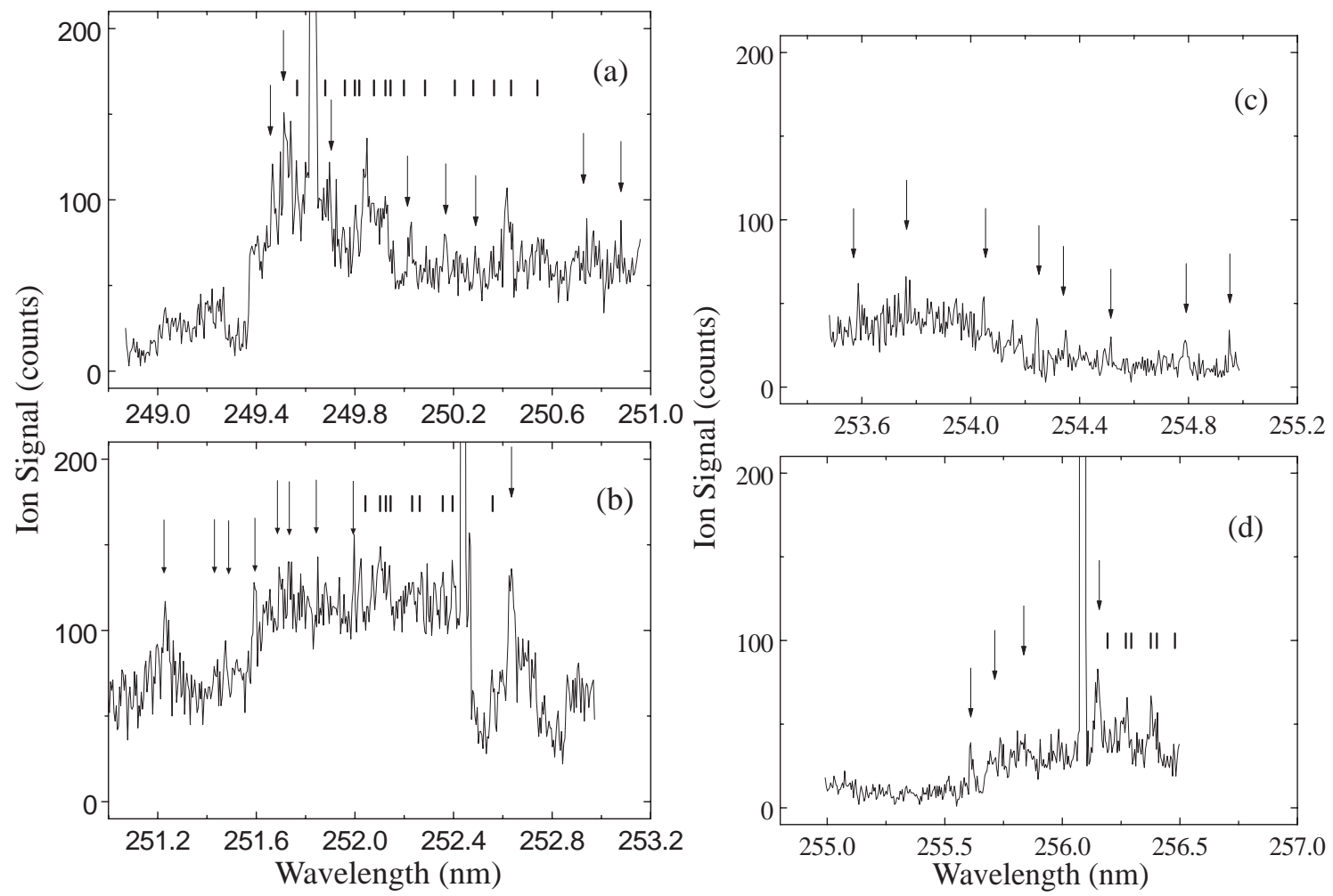

Fig. 4.6 Excitation spectra of 0.5\% Xe in Ar.

${ }^{21}$ S. T. Pratt, P. M. Dehmer, and J. L. Dehmer, J. Chem. Phys. 96, 2099(1992) 
The new lines appear on both the blue and red sides of the resonances. An interesting band of transitions appears around $251.2 \mathrm{~nm}$, a band which, even though blue shifted in this case, was observed in pure xenon and xenon-helium mixtures, and is thought to dissociate to the $5 \mathrm{~d}[1 / 2]_{1}$ level. This band is shown in Fig. 4.7 in pure $\mathrm{Xe}$ and $\mathrm{Xe} / \mathrm{He}$ at different laser delay times resulting in diffent temperatures in the expansion. Intensities varied significantly suggesting different processes of clustering and dissociation.

Spectra taken for Xe/Ar mixtures under different conditions (Figure 4.8) show large changes of the intensities of various lines. Mixtures of $0.5 \% \mathrm{Xe}$ in $\mathrm{Ar}$ and $5 \% \mathrm{Xe}$ in $\mathrm{Ar}$ are compared at 2200 torr stagnation pressure and $210 \mathrm{~K}$ stagnation temperature. As observed, the formation of the clusters occurs a large time after the nozzle is first opened. By adjusting the delay time between the beginning of the molecular pulse and the beginning of the laser pulse, one can find the time for the formation of clusters. For the marked excitation wavelengths on Figure 4.8(b), TOF spectra were taken and are shown in Fig. 4.9. Clearly, the peak at 2498.9A is a $\mathrm{Xe}_{2}$ resonance. The broad $\mathrm{Xe}$ and $\mathrm{Xe}_{2}{ }^{+}$peaks in the TOF (Fig. 4.9(e), (f)) suggest the existence of heteroclusters of Xe and Ar, e.g., $\mathrm{XeAr}_{\mathrm{n}}$ and $\mathrm{Xe}_{2} \mathrm{Ar}_{\mathrm{n}}$.

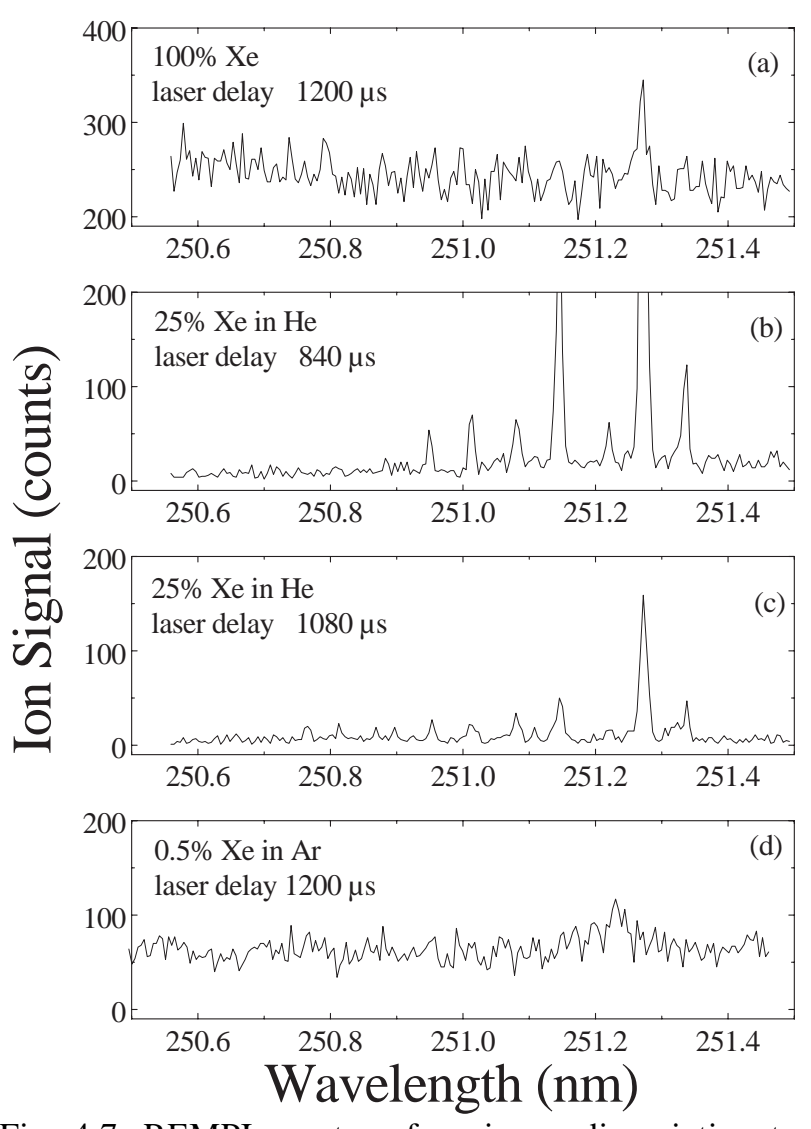

Fig. 4.7. REMPI spectra of excimers dissociating to $5 \mathrm{~d}[1 / 2] 1$

One can observe in Figure 4.8(a) and (c) that the peaks at $249.89 \mathrm{~nm}$ disappear at low concentration of Xe to leave only the peaks at $249.48 \mathrm{~nm}, 249.53 \mathrm{~nm}$ and $249.70 \mathrm{~nm}$ which were assigned to the

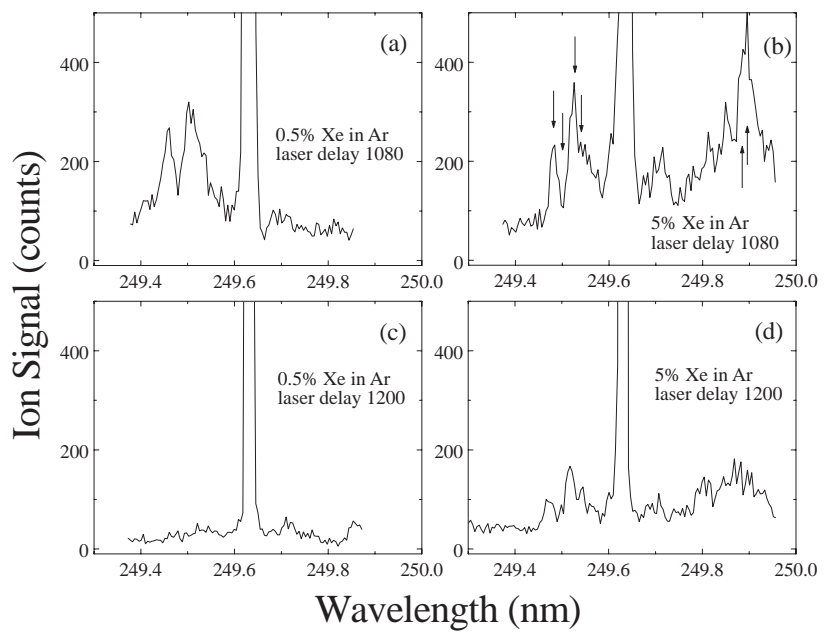

Fig. 4.8 REMPI spectra for various expan-sion times and mixtures. Delay times are in $\mu$ s.

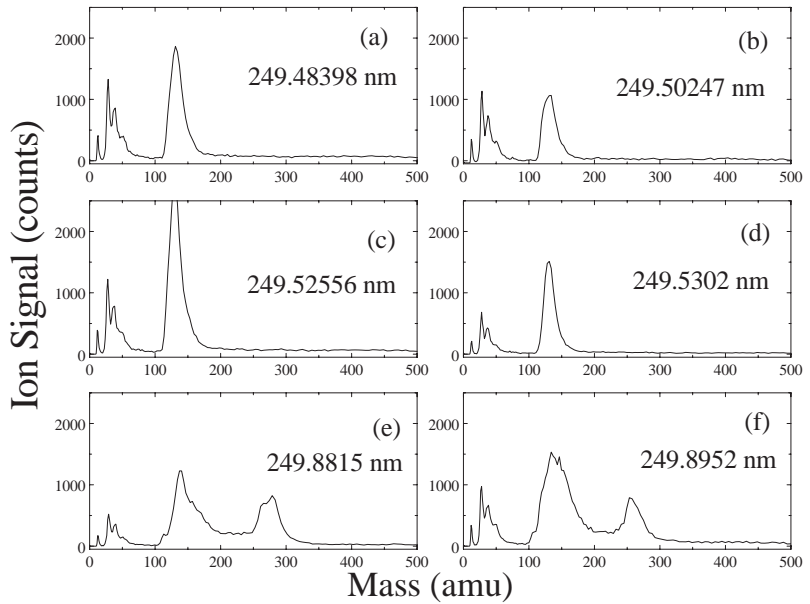

Fig. 4.9. TOF spectra for various wavelength of Fig. 3.4 
Xe-Ar dimer. At later laser delay times all clusters of peaks (even though weaker) are present for the higher concentration of Xe and nearly disappear for lower concentrations of Xe. As measured for XeHe clusters , the formation of "magic number" clusters is favored. The number of their atoms can be connected with structures considered to be particularly stable. For the Xe-He clusters, such structures were $\mathrm{Xe}_{2} \mathrm{He}_{6}$ and $\mathrm{Xe}_{2} \mathrm{He}_{13}$. For the Xe-Ar clusters, such structures are expected to be $\mathrm{Xe}_{2} \mathrm{Ar}_{5}$ and $\mathrm{Xe}_{2} \mathrm{Ar}_{11}$. In Figure 4.10 we present a blowup of Figure 4.9e. One can see a small peak at mass $460 \mathrm{amu}$, which is assigned to the $\mathrm{Xe}_{2} \mathrm{Ar}_{5}$ cluster. Going to lower temperatures (which facilitates clustering) might increase the intensity this peak, along with resolving the masses in the broadened $\mathrm{Xe}^{+}$and $\mathrm{Xe}_{2}{ }^{+}$peaks.

\subsubsection{Conclusions}

We presented experiments on resonantly enhanced multiphoton ionization and time-offlight spectroscopy of Xe-Ar mixtures, expanded in a pulsed supersonic jet. By comparing the spectra for different mixtures of xenon and argon we were able to distinguish the lines coming from the xenon-argon dimers and Xe$\mathrm{Ar}_{\mathrm{n}}$ clusters.

Our spectra show new peaks, not observed in the spectra of the Xe-Ar dimer by Pratt et al. We conclude that these features are due to the formation of larger $\mathrm{Xe}^{-\mathrm{Ar}_{\mathrm{n}}}$ clusters that form at higher pressures and lower temperatures.

Though these results are preliminary, we

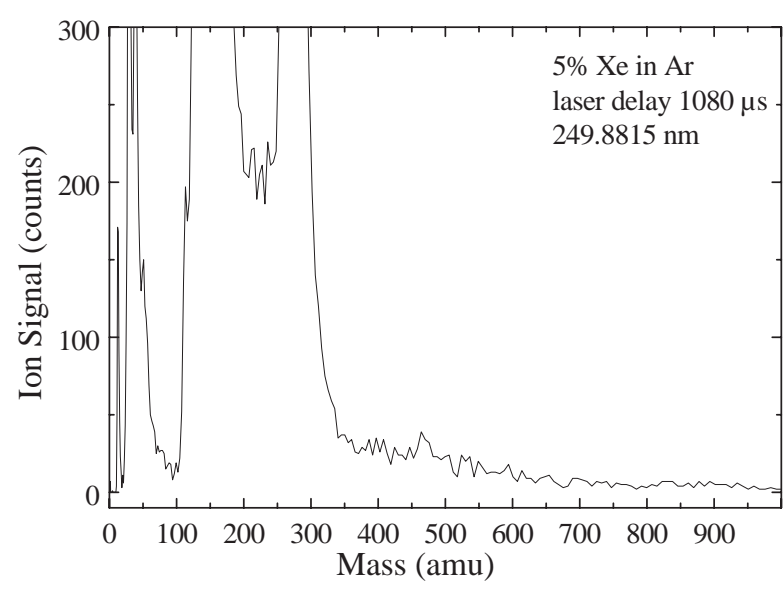

Fig. 4.10 TOF spectrum showing the $\mathrm{Xe}_{2} \mathrm{Ar}_{5}$ cluster.

have defined regions for additional study. We have found that lower temperatures sharpen the mass spectra and additional excitation spectra will be more mass specific. The initial conditions (stagnation pressure and stagnation temperature) of the gas expansion are crucial in formation of clusters of predetermined size. 


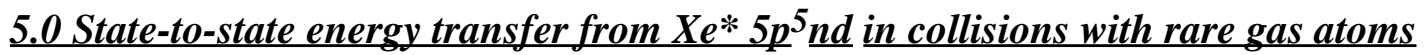

\subsection{Introduction}

In the nuclear pumped atomic xenon laser molecular ions are formed by association of atomic ions produced by the high energy particles and hot secondary electrons. Dissociation recombination of the these molecular ions produce excited atoms in the $5 p^{5} 7 \mathrm{p}$ and $5 \mathrm{p}^{5} 6 \mathrm{~d}$ manifolds. Collisions then populate the upper laser state, e.g.. $5 \mathrm{~d}[1 / 2]_{2} \rightarrow 6 \mathrm{p}[5 / 2]_{2}\left(3 \mathrm{~d}_{5} \rightarrow 2 \mathrm{p} 9\right)$ at $1.73 \mu \mathrm{m}),{ }^{22}$ Collisions deplete the lower laser state to states of Xe* $6 \mathrm{~s}$ and $6 \mathrm{~s}^{\prime}$. These states have long lifetimes either because they are metastable, or for the resonance states, radiation is trapped. Electron impact excitation can promote these states to the $5 \mathrm{~d}, 7 \mathrm{~s}, 7 \mathrm{p}$, and $6 \mathrm{~d}$ states where collisional cascade can again repopulate the upper laser state. Because of this circulation of excited state population the laser can operate efficiently.

\section{Xenon - Two Laser Experiment}

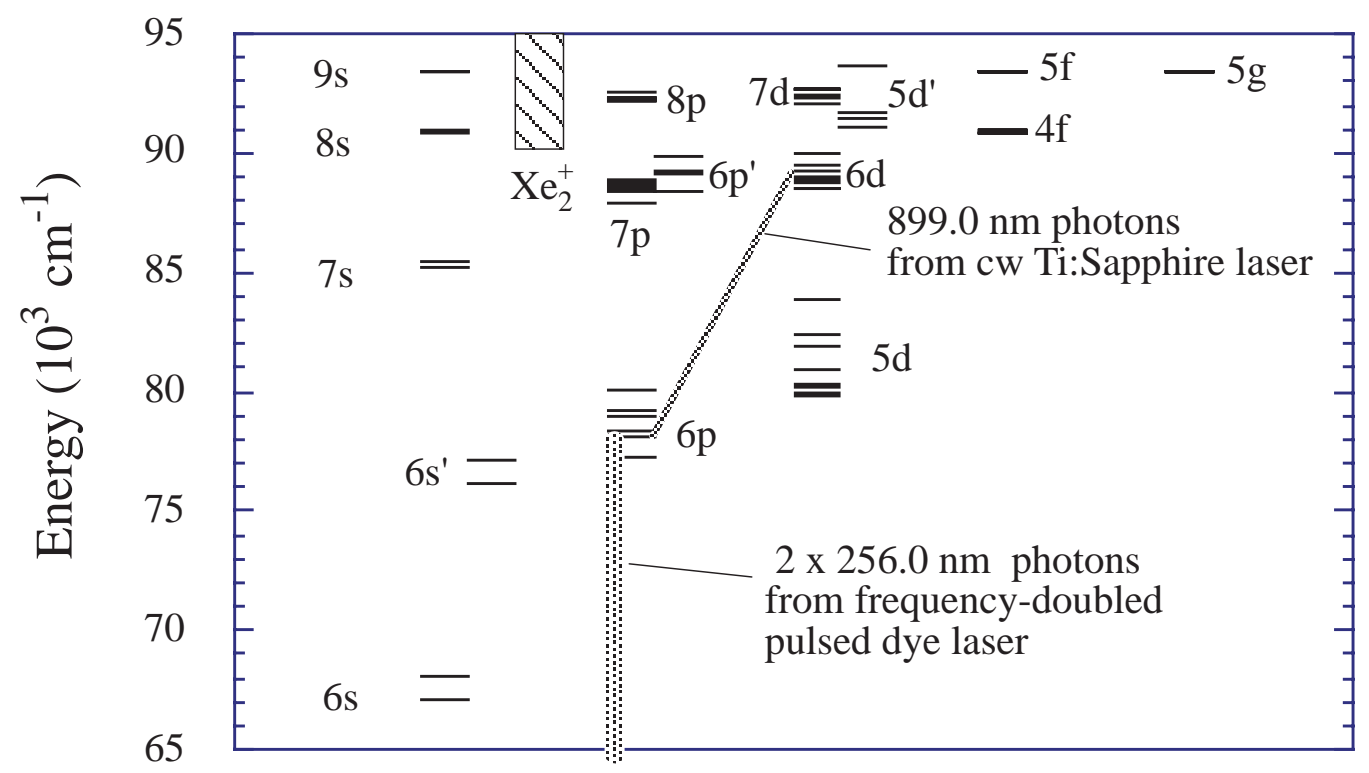

Fig. 5.1 Term diagram showing $5 \mathrm{p}^{5} \mathrm{nl}$ multiplets of xenon. A two-color experiment for excitation of $\mathrm{Xe}^{*} 6 \mathrm{~d}[5 / 2]_{2}$ is shown.

As observed in Fig. 5.1, many states in the 7p, 6p' 6d, 7s, 6p and 5d can participate in collisional deactivation to the lasing transition. Previously several research groups have studied collisional energy transfer in the $5 \mathrm{p}^{5} 6 \mathrm{p}$ manifold. ${ }^{23,24,25,26}$ The lower energy states of this manifold are

${ }^{22}$ M. Ohwa, T. J. Moratz, and M. J. Kushner, J. Appl. Phys. 66, 5131 (1989).

${ }^{23}$ N. Bowering, M. R. Bruce and J.W. Keto, "Collisional Deactivation of Two-Photon Excited Xenon 5 556 Atoms: I. State-to-State Reaction Rates," J. Chem. Phys. 84, 709-714 (1986) and "Collisional Deactivation of Two-Photon Excited Xenon 5p $6 \mathrm{p}$ Atoms: II. Lifetimes and Quench Rates," J. Chem. Phys. 84, 715-726 (1986);.

${ }^{24}$ M. R. Bruce, W. B. Layne, C. A. Whitehead, and J. W. Keto, J. Chem. Phys. 92, 2917 (1990).

25 Horiguchi, R. S. F. Chang, and D. W. Setser, J. Chem. Phys. 96, 2099 (1981); J. Xu and D. W. Setser, J. Chem. Phys. 92, 4191 (1990).

${ }^{26}$ W. J. Alford, IEEE J. Quant. Elect. 26, 1633 (1990); J. Chem. Phys. 96, to be published. 
populated by the lasing transition, hence collisional excitation and destruction of these states effect the population inversion and hence the gain of the laser. If collisions do not rapidly deplete these states, the population inversion will terminate early in the discharge.

We studied collisional deactivation of these excited manifolds in mixtures of rare-gases. In addition to contributing to the understanding of the laser, these collisions are important to other systems using discharges of rare gases, such as discharges for plasma etching and lamps.

Table 5.1. Paschen and Racah notation for states of Xe 5s,6p, and 5d.

\begin{tabular}{ll}
\hline Pachen notation & Racah notation \\
\hline $2 \mathrm{p}_{5}$ & $6 \mathrm{p}[1 / 2]_{0}$ \\
$2 \mathrm{p}_{6}$ & $6 \mathrm{p}[3 / 2]_{2}$ \\
$2 \mathrm{p}_{7}$ & $6 \mathrm{p}[3 / 2]_{1}$ \\
$2 \mathrm{p}_{8}$ & $6 \mathrm{p}[5 / 2]_{3}$ \\
$2 \mathrm{p}_{9}$ & $6 \mathrm{p}[5 / 2]_{2}$ \\
$2 \mathrm{p}_{10}$ & $6 \mathrm{p} 1 / 2]_{1}$ \\
$3 \mathrm{~d}_{2}$ & $5 \mathrm{~d}[3 / 2]_{1}$ \\
$3 \mathrm{~d}_{3}$ & $5 \mathrm{~d}[3 / 2]_{2}$ \\
$3 \mathrm{~d}_{4}$ & $5 \mathrm{~d}[7 / 2]_{3}$ \\
$3 \mathrm{~d}_{5}$ & $5 \mathrm{~d}[1 / 2]_{1}$ \\
$3 \mathrm{~d}_{6}$ & $5 \mathrm{~d}[1 / 2]_{0}$ \\
$1 \mathrm{~s}_{2}$ & $6 \mathrm{~s}^{\prime}[1 / 2]_{1}$ \\
$1 \mathrm{~s}_{3}$ & $6 \mathrm{~s}^{\prime}[1 / 2]_{0}$ \\
\hline
\end{tabular}

The measured reaction rates can be compared with recent calculations of state-to-state energy transfer in rare-gases by Hickman, Huestis, and Saxon (HHD). ${ }^{27}$ These calculations start with a fully ab-initio calculation using relativistic Hartree-Fock for the ion cores and a full CI calculation for the valence electrons. The accuracy of the calculation is then improved using a semiempirical approach to generate a pseudopotential for the xenon ion core. Many of the calculated matrix elements agree remarkably with experiment; but also contain interesting disagreements that may point to weaknesses in the current experiments. The potentials and matrix elements give a qualitative insight why reaction rates differ greatly for adjacent states; and they contain new information suggesting new experiments which should provide information and deeper insight into this complex area of research.

Comparisons between the calculations and experiment for total quench rate and state-to-state reaction rates vary between states 28 . Different groups use different spectroscopic notations to describe the atomic levels. HHD use primarily Paschen notation which numbers the states in ascending order within a multiplet as the energy decreases. We have more commonly described them in Racah notation, which defines the quantum numbers for intermediate angular momentum coupling, $\mathrm{nL}\left[\mathrm{K}=\mathrm{J}_{\text {core }}+\mathrm{s}\right] \mathrm{J}$. We compare the notations in Table 5.1. Calculations for total quench

${ }^{27}$ A. P. Hickman, D. L. Huestis, and R. P. Saxon, "Interatomic Potentials for Excited States of XeHe and XeAr", J. Chem. Phys. 98, 5419(1993).

${ }^{28}$ A. P. Hickman, D. L. Huestis, and R. P. Saxon, "Calculations of inelastic collisions of excited states of xe with He and Ar", J. Chem Phys. 96, 2099(1992). 
reaction rates for $\mathrm{Xe}^{*} 2 \mathrm{p}_{6} 1 \mathrm{~s}_{2}, 2 \mathrm{p}_{10}$, and $3 \mathrm{~d}_{5}$ in argon agree to within approximately $\pm 30 \%$ with our previous measurements ${ }^{29}$ and measurements by Setser et. al. ${ }^{30}$ and Alford ${ }^{31}$.

Experiments are lacking for reactions of $X e^{*} 5 d: 3 d_{3}, 3 d_{4}, 3 d_{5}$, and $3 d_{6}$. Because $5 d$ states of xenon radiate to states of $\mathrm{Xe}^{*} 6 \mathrm{p}$ in the infrared at wavelengths beyond those detectable with sensitive photo detectors $(\lambda>1 \mu \mathrm{m})$, few experiments exist to date. We have observed $3 \mathrm{~d}_{5}$ indirectly though the fluorescence of $2 \mathrm{p}_{5}$, which is mixed rapidly with $3 \mathrm{~d}_{5}$ in argon buffers. By fitting the resulting two exponential time decays as a function of pressure, we measured the state-tostate mixing rates and total quench rates for both states. We confirmed this mixing with $3 \mathrm{~d}_{5}$ by independently observing the fluorescence of $3 \mathrm{~d}_{5}$ in the vacuum ultraviolet (VUV) $3 \mathrm{~d}_{5} \rightarrow 5 \mathrm{p}^{6}$ at 125 $\mathrm{nm} .{ }^{32}$ Because of the radiation trapping of the resonance fluorescence, signals were weak and we could observe the time dependence only over a narrow pressure range. For low pressures, collisional production rates for the state are too slow; for high pressures the trapped radiation doesn't compete with the IR fluorescence at $1.73 \mu \mathrm{m}$. Collision processes involving $6 \mathrm{p}^{5} 5 \mathrm{~d}$ require a method of direct excitation or detection for these states.

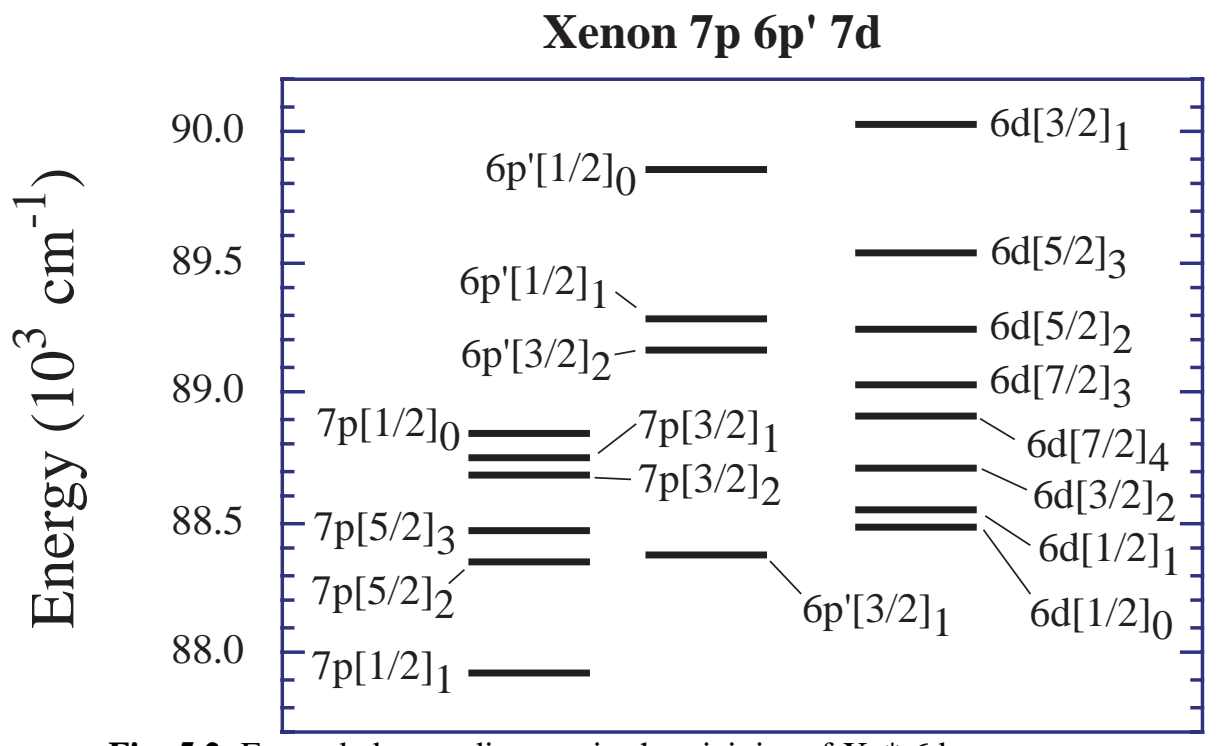

Fig. 5.2. Expanded term diagram in the vicinity of $\mathrm{Xe}^{*} 6 \mathrm{~d}$.

We previously measured quenching rates for excited atoms in the $7 \mathrm{p}$ and $6 \mathrm{p}^{\prime}$ manifolds in an argon buffer gas 33 and state-to-state reaction rates in xenon and krypton buffers. ${ }^{34}$ Collisional deactivation of these states should be important in formation of the upper laser state in $5 \mathrm{p}^{5} 5 \mathrm{~d}$. We expected to observe bright fluorescence from Xe* $5 \mathrm{p}^{5} 6 \mathrm{~d}$ since these states are in near resonance with $\mathrm{Xe}^{*} 5 \mathrm{p}^{5} 7 \mathrm{p}$ (see and expansion of this energy range in Fig. 5.2). Setser observed rapid

${ }^{29}$ M. R. Bruce, W. B. Layne, C. A. Whitehead, and J. W. Keto, J. Chem. Phys. 92, 2917 (1990).

${ }^{30}$ Horiguchi, R. S. F. Chang, and D. W. Setser, J. Chem. Phys. 96, 2099 (1981); J. Xu and D. W. Setser,

J. Chem. Phys. 92, 4191 (1990).

${ }^{31}$ W. J. Alford, IEEE J. Quant. Elect. 26, 1633 (1990); J. Chem. Phys. 96, to be published.

${ }^{32}$ M. R. Bruce, et. al. ibid.

33 M. R. Bruce, et. al., ibid.

${ }^{34}$ C. A. Whitehead, M. R. Bruce, Hong Cai, J. Kohel, W. B. Layne, and J. W. Keto,"Deactivation of TwoPhoton Excited $\mathrm{Xe}\left(5 p^{5} 6 p, 6 p^{\prime}, 7 p\right)$ and $\mathrm{Kr}\left(4 p^{5} 5 p\right)$ in Xenon and Krypton", J. Chem. Phys.102,1965(1995) 
collisional deactivation in argon and neon buffers, ${ }^{35}$ but in xenon and krypton buffers we observed no collisional deactivation to states of $5 \mathrm{p}^{5} 6 \mathrm{~d}$. We observed collisional product fluorescence only from states of $\mathrm{Xe}^{*} 5 \mathrm{p}^{5} 6 \mathrm{p}$, primarily from $\mathrm{Xe}^{*} 5 \mathrm{p}^{5} 6 \mathrm{p}[1 / 2]_{0}$. From the time dependence of the product fluorescence, we showed that this process was indirect. We tentatively identified the intermediate states as belonging to $\mathrm{Xe}^{*} 5 \mathrm{~d}$.

Since the states of $\mathrm{Xe}^{*} 6 \mathrm{~d}$ are near resonant to $\mathrm{Xe} * 7 \mathrm{p}$, it is a mystery that they are not produced by collisions. A study of $\mathrm{Xe}^{*} 5 \mathrm{p}^{5} 6 \mathrm{~d}$ excited selectively would help resolve this mystery. We proposed to study reactions of $\mathrm{Xe}^{*} 5 \mathrm{p}^{5} 5 \mathrm{~d}$ and $5 \mathrm{p}^{5} 6 \mathrm{~d}$ using two-color experiments. First a UV laser is used to excited states of $\mathrm{Xe}^{*} 5 \mathrm{p}^{5} 6 \mathrm{p}, 6 \mathrm{p}^{\prime}$, or $7 \mathrm{p}$ in a two-photon transition and then an infrared laser will be used to either probe populations of $\mathrm{Xe}^{*} 5 \mathrm{p}^{5} 5 \mathrm{~d}$ produced by collisions or the infrared (IR) laser will be used to excited states of Xe* $5 \mathrm{p}^{5} 6 \mathrm{~d}$ selectively.

\subsection{State-to-state collision studies of $\mathrm{Xe}^{*} 5 \mathrm{p}^{56 \mathrm{~d}}$ multiplets using a two-color experiment}

We have observed $\mathrm{Xe}^{*} 5 \mathrm{~d}[1 / 2]_{1}$ indirectly though the fluorescence of $6 \mathrm{p}[1 / 2]_{0}$ which is mixed rapidly with $5 \mathrm{~d}[1 / 2]_{1}$ in argon buffers, and we confirmed this mixing with $3 \mathrm{~d}_{5}$ by independently observing the fluorescence of $5 \mathrm{~d}[1 / 2]_{1}$ in the vacuum ultraviolet (VUV) $3 \mathrm{~d}_{5} \rightarrow 5 \mathrm{p}^{6}$ at $125 \mathrm{~nm} .{ }^{36}$ To observe this state directly we attempted several two-color experiments.

We first excited $\mathrm{Xe}^{*} 6 \mathrm{p}[1 / 2]_{0}$ in a two-photon transition

$$
\mathrm{Xe}(5 \mathrm{p})^{6}+2 \mathrm{~h} \omega(\lambda=249.6272 \mathrm{~nm}) \rightarrow \mathrm{Xe}^{*} 6 \mathrm{p}[1 / 2]_{0}
$$

This excited state then undergoes collisional deactivation to $5 \mathrm{~d}[1 / 2]_{1}$

$$
\mathrm{Xe} * 6 \mathrm{p}[1 / 2]_{0}+\mathrm{Ar} \leftrightarrow \mathrm{Xe}^{*} 5 \mathrm{~d}[1 / 2]_{1} .
$$

We attempted several approaches for the detection of $5 \mathrm{~d}[1 / 2]_{1}$ using laser induced fluorescence (LIF). One set of experiments searched for excited states accessible to light with wavelengths within the operating range of a Ti-sapphire laser. In an example approach we tried exciting to states of $\mathrm{Xe} * 4 \mathrm{f}$ or $5 \mathrm{f}: 37,38$

$$
\begin{aligned}
& \mathrm{Xe}^{*} 5 \mathrm{~d}[1 / 2] 1+\mathrm{h} \omega(\lambda=747.6 \mathrm{~nm}) \rightarrow 5 \mathrm{f}[3 / 2] 1 \\
& 5 \mathrm{f}[3 / 2] 1 \rightarrow 6 \mathrm{~s}[3 / 2] 1+\mathrm{h} \omega\left(\lambda=582.5 \mathrm{~nm}, \mathrm{f}_{\mathrm{ij}}=0.62\right) .
\end{aligned}
$$

${ }^{35}$ Gen Inoue, J. K. Ku, and D. W. Setser, J. Chem. Phys. 81, 5760(1984).

${ }^{36}$ M. R. Bruce, et. al. ibid.

37John C. Miller, Phys. Rev. A40, 6969(1989).

38 C. J. Humphreys, Bureau of Standards of Research 5, 1041(1930). 
This sequence is illustrated in Fig. 5.3. Due to collisional mixing between $\mathrm{Xe} 6 \mathrm{p}[1 / 2] 0$ and $\mathrm{Xe}$ $5 \mathrm{~d}[1 / 2] 1$ at 8 Torr of buffer pressure, $10 \mathrm{nsec}$ after two-photon excitation of $6 \mathrm{p}[1 / 2] 0,30 \%$ of the atoms decay to $5 \mathrm{~d}[1 / 2] 1$. Because of strong configuration mixing, the $4 \mathrm{f}$ and $5 f$ states have strong transition rates ${ }^{39}$ to $\mathrm{Xe}^{*} 5 \mathrm{~d}$ and 6 s states. The radiative branching fraction for the $5 \mathrm{f}[3 / 2] 1$ state to radiate at $582.5 \mathrm{~nm}$ is $62 \%$. The CW Tisapphire laser had sufficient intensity to saturate the transition. Asumming no significant quenching we estimated that we would observe 8.5 photons per laser shot from $5 \mathrm{f}[3 / 2]_{1}$. Given the sensitivity $(\sim 2$ x $10^{-3}$ photons/laser shot) of our detection system at this wavelength, we should have tolerated a significant quenching rate and still observed a signal. We have also tried states of $\mathrm{Xe}^{*} 5 \mathrm{p}^{5} 8 \mathrm{p}$. Unfortunately, none of these experiments were successful. We speculate that because these states lie above the $\mathrm{Xe}_{2}{ }^{+}$ionization threshold that they are rapidly quenched by associative ionization, preventing observable fluorescence. Other choices for direct detection of $\mathrm{Xe}^{*} 5 \mathrm{~d}$ require longer wavelength sources.

One possible set of states are Xe* $6 \mathrm{p}^{\prime}$. The transition $5 \mathrm{~d}[1 / 2] 1 \rightarrow 6 \mathrm{p}^{\prime}[1 / 2]_{0}$ occurs at 1012.8 $\mathrm{nm}$, the very edge of operation for our Ti-sapphire laser (To reach this wavelength the argon ion pump laser must operated above 15 Watts with very good mode quality. Our laser could only accomplish this early in the warranty period for the laser tube. The $6 \mathrm{p}^{\prime}[1 / 2]_{0}$ state has a relatively short radiative lifetimes and a large branching fraction in the near infrared where our IR PMTs are efficient. We attempted to build an infrared laser operating reliably with wavelength greater than $1000 \mathrm{~nm}$. This would have enabled the detection of more energetic states of Xe* 5d.

A promising candidate was the room temperature color-center laser $\left(\mathrm{LiF}: \mathrm{F}_{2}^{+}\right)$pumped by the $532 \mathrm{~nm}$ output of our Nd:Yag. Data provided by the Russian company from whom we purchased the crystal reported lasing over wavelengths from 700 to $1200 \mathrm{~nm}$ when using a Ruby laser as the pump source. We constructed a longitudinal pumped oscillator, grating tuned in Littrow, and amplified by pumping the back side of the crystal $(1 \mathrm{~cm} \times 2 \mathrm{~cm} \times 5 \mathrm{~cm})$. While this laser worked well, we were only able to tune the laser from 880 to $1000 \mathrm{~nm}$ with output energies of a few mJ per pulse. This wavelength was too short for direct excitation of states of $\mathrm{Xe}^{*} 5 \mathrm{~d}$ and thus represented no advantage over our Ti-sapphire laser.

${ }^{39}$ A. V. Loginov and P. F. Gruzdev, Opt. Spectrosc. 41, 104(1976). 


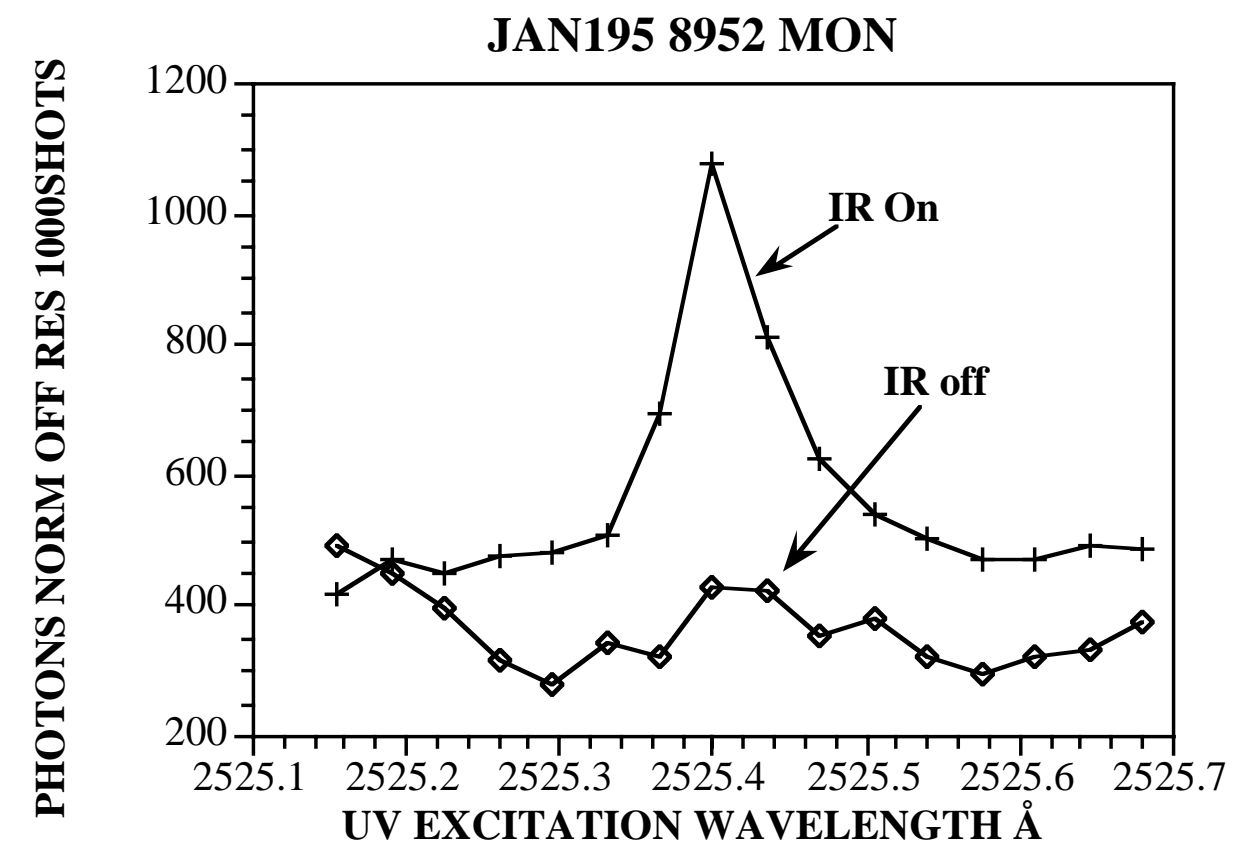

Fig. 5.4. Two- photon excitation scan of $6 \mathrm{p}[3 / 2] 2$ while monitoring the fluorescence of a collision product at $895.2 \mathrm{~nm}$. When the IR laser is off most of the excited population decays by fluorescence, and the collision product is unobservable. With the IR laser on, the state is effectively prevented from fluorescence.

Additional difficulties were overcome for these experiments. The beams must overlap both in time and space and both lasers must be tuned accurately to the correct wavelengths. To minimize alignment uncertainty, we have used IR beams with large diameters at the focus which overlap and counter propagate relative to the exciting UV laser beam. For a new series of experiments, we have used the $6 \mathrm{~s}[3 / 2] 2$ state, which is filled by fluorescence of $6 \mathrm{p}[3 / 2] 2$ at $823.4 \mathrm{~nm}$ radiation, to align the beams. By re pumping the $6 \mathrm{~s}[3 / 2] 2$ state to $6 \mathrm{p}[3 / 2] 2$ with the IR laser, we effectively prevent it from decaying by fluorescence. A resulting spectrum is shown in Fig. 5.4 where we observe the fluorescence from $6 s[3 / 2]_{2}$ in a transition to $6 s[3 / 2]_{1}$ at a long wavelength. When the laser is tuned onto resonance, the fluorescence of this transition becomes brighter because a greater fraction of population emits in this transition than when the laser is off. 
By maximizing this signal we were assured of maximal overlap between the UV and IR lasers. To minimize uncertainty in wavelength of our probe radiation, we constructed an optogalvanic discharge cell. Using this cell, $\mathrm{Xe}^{*} 5 \mathrm{~d}$ (and other) states are excited by the discharge to an equilibrium population. Introducing the IR probe light at a transition disturbs this population distribution. In most cases, this relatively small population change results in a measurable change in the discharge impedance. This laser optogalvanic effect has been used for both calibration and stabilization. An oscilloscope with some simple circuits was used to rapidly find a given resonance. Lock-in detection was then used to give detailed spectra as observed in Fig. 5.5. The strength and shape (and polarity) of the optogalvanic spectra can depend other factors, such as the geometry of the discharge.

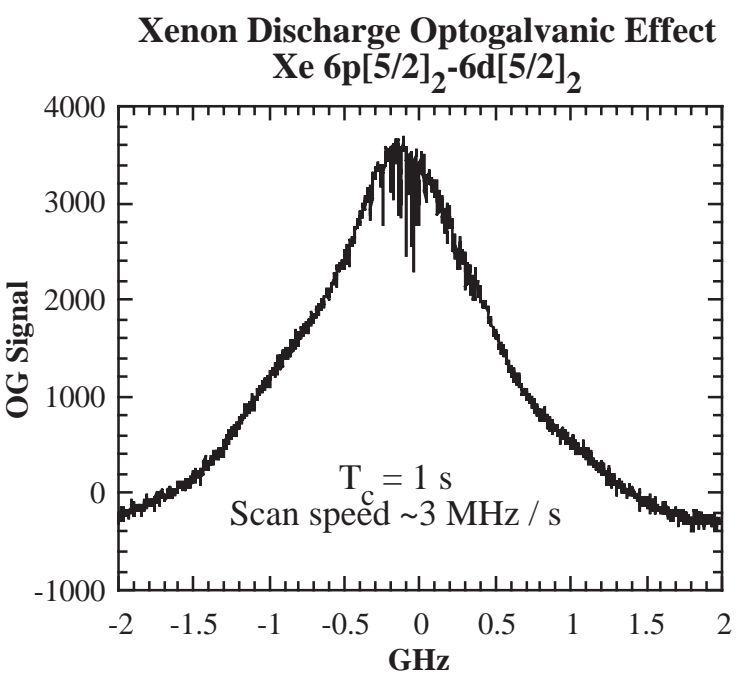

Fig. 5.5. Optogalvanic signal as a function of the IR laser frequency.

\subsection{Experimental studies of the $5 p^{5} 6 \mathrm{~d}$ multiplets}

To selectively excite states in the $6 \mathrm{~d}$ manifold, $\mathrm{Xe} * 6 \mathrm{p}[5 / 2] 2$ was first excited in a two-photon transition followed by excitation to $6 \mathrm{~d}[7 / 2] 3,6 \mathrm{~d}[5 / 2] 2,6 \mathrm{~d}[5 / 2] 3,6 \mathrm{~d}[3 / 2] 1$ from $6 \mathrm{p}[5 / 2] 2$ using a Ti:Sapphire laser (currently a Coherent model 899 with mid-wave optics). With long-wave optics, the $6 \mathrm{~d}[7 / 2] 3,6 \mathrm{~d}[3 / 2] 2$ and $6 \mathrm{~d}[1 / 2] 1$ states can be excited. This is shown schematically in Fig. 5.1.

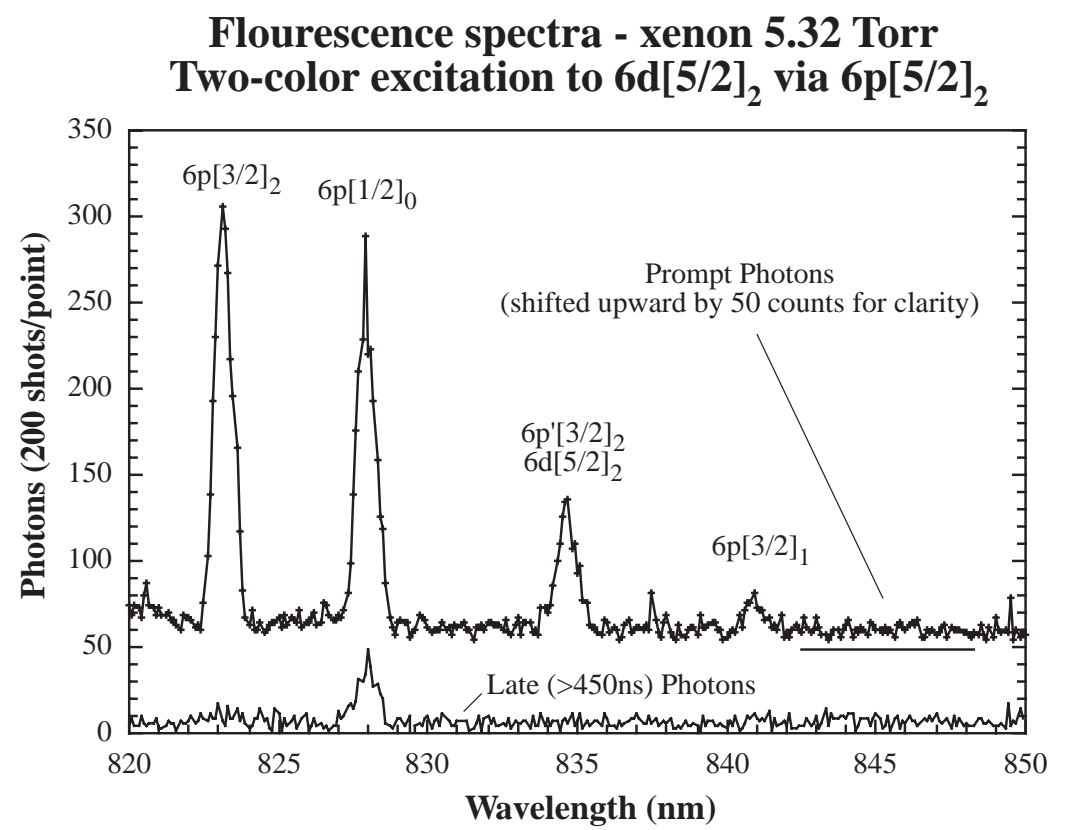

Fig. 5.6. Scan of fluorescence wavelength when selectively exciting $\mathrm{Xe} * 6 \mathrm{~d}[5 / 2] 2$. The prompt photons are counted in a time window from 0 to $450 \mathrm{~ns}$. The transitions are labeled by their excited state.

Figure 5.6 is a monochrometer scan of the fluorescence produced by two-color excitation. As described above, $\mathrm{Xe}^{*} 6 \mathrm{p}[5 / 2] 2$ was populated via a two-photon transition by a short (6 ns), 
relatively intense $(\sim 1 \mathrm{~mJ})$ laser pulse at $256 \mathrm{~nm}$. The CW Ti:Sapphire laser is tuned to the peak of the $6 \mathrm{p}[5 / 2] 2-6 \mathrm{~d}[5 / 2] 2 \quad(\sim 899.015 \mathrm{~nm}$ vacuum $)$ optogalvanic resonance. We observed fluorescence from $6 \mathrm{p}[3 / 2]_{2}, 6 \mathrm{p}[1 / 2]_{0}$, and $6 \mathrm{p}[3 / 2]_{1}$. The peak at $835 \mathrm{~nm}$ may be fluorescence from either $6 \mathrm{p}^{\prime}[3 / 2]_{2}$, $6 \mathrm{~d}[5 / 2]_{2}$, or both; unfortunately the direct $\mathrm{Xe} * 6 \mathrm{~d}[5 / 2] 2$ fluorescence has not yet been convincingly isolated spectrally from the adjacent $6 p^{\prime}[3 / 2] 2$ state. The weak $6 \mathrm{~d}[5 / 2]_{2}$ fluorescence indicates rapid quenching of the directly excited state. Notice that the $6 \mathrm{p}[1 / 2]_{0}$ line has a large (20\%) late time ( $>450 \mathrm{~ns}$ ) component. Since it can not be directly populated by radiation from $6 \mathrm{~d}[5 / 2] 2$ $(j=2$ to $j=0)$, light from $6 p[1 / 2]_{0}$ must result from

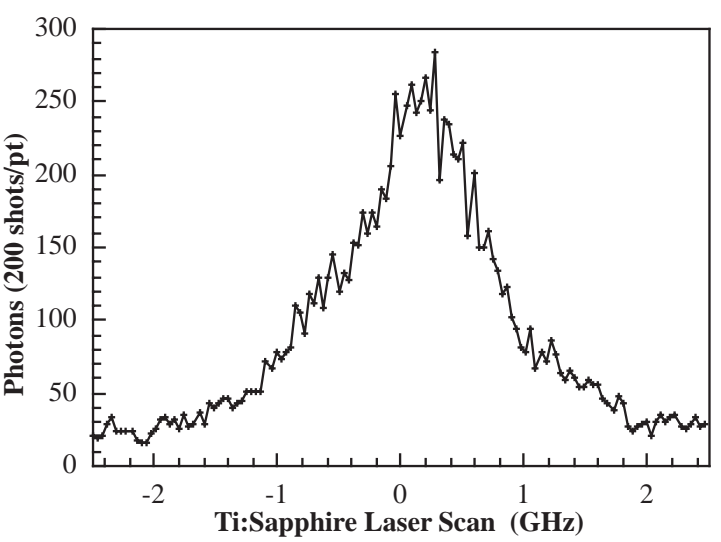
collisions. The late time component is similar to our previous observations for the time dependence of $\mathrm{Xe}^{*} 7 \mathrm{p}[5 / 2]_{2} \rightarrow \mathrm{Xe}^{*} 5 \mathrm{~d} \rightarrow \mathrm{Xe} * 6 \mathrm{p}[1 / 2]_{0}$. Xe*

Fig. 5.7. Two-color excitation of $6 \mathrm{~d}[5 / 2] 2$ as a function of the frequency of the infrared laser. The fluorescence was monitored from $\mathrm{Xe}^{*} 6 \mathrm{p}[1 / 2] 0$ excited as a collision product. The xenon pressure was 5.32 Torr.

$5 \mathrm{~d}[3 / 2]_{1}$ has a theoretical ${ }^{40}$ radiative lifetime of 245

ns with a strong branching fraction to $\mathrm{Xe}^{*} 6 \mathrm{p}[1 / 2]_{0}$. In experiments here, excitation transfer from $\mathrm{Xe} * 6 \mathrm{~d}[5 / 2] 2$ may proceed by collisional deactivation to $\mathrm{Xe} * 5 \mathrm{~d}[3 / 2]_{1}$ followed by fluorescence to $\mathrm{Xe}^{*} 6 \mathrm{p}[1 / 2]_{0}$. The Ti:Sapphire laser is narrow band $(\sim 1 \mathrm{MHz})$ and can be finely scanned over line width of the $6 \mathrm{p}[5 / 2] 2-6 \mathrm{~d}[5 / 2] 2$ transition, an example spectrum is shown in Fig. 5.7.

By measuring the linewidths for laser excitation and the total fluorescence intensities of the $6 \mathrm{~d}[5 / 2]_{3}$ with the simultaneous measure of the intensity of the $6 \mathrm{p}[5 / 2]_{2}$ coupled to the upper state with the laser on and off, all measured as a function of pressure, the quench rate of the upper state could be determined. ${ }^{41}$ The quench rate for $6 \mathrm{~d}[5 / 2]_{3}$ was measured to be $7.5 \pm 0.810^{-10} \mathrm{~cm}^{3} \mathrm{sec}^{-1}$, which is larger than the larges rate measured for the nearby $7 \mathrm{p}$ manifold, $7 \mathrm{p}[3 / 2] 2$ with kquench $=$ $5.22 \pm 0.0810^{-10} \mathrm{~cm}^{3} \mathrm{sec}^{-1}$. The collisional product branching fraction from the $6 \mathrm{~d}$ state was measured to be $0.56,0.32$, and 0.12 for $6 \mathrm{p}[3 / 2]_{2}, 6 \mathrm{p}[3 / 2]_{1}$, and $6 \mathrm{p}[1 / 2]_{0}$ respectively. Measurements for $6 \mathrm{~d}[5 / 2] 2$ were not possible because fluorescence from this state could not be seen because of rapid quenching to the adjacent $\left(80.9 \mathrm{~cm}^{-1}\right.$ below) $6 \mathrm{p}^{\prime}[3 / 2]_{2}$ state. Only product state fluroescence from quenching was seen as shown in Fig. 5.6.

${ }^{40}$ M. Aymar and M. Coulombe, At. Data Nucl. Data Tables 21, 537 (1978).

${ }^{41}$ Joe P. Holder, Two-collor studies of xenon $5 p^{5} 5 d$ and $5 p^{5} 6 d$ multiplets in rare gas buffers, Ph. D. Thesis, The University of Texas at Austin, 1997. 


\subsection{Laser assisted half-collisions in reactions of $\mathrm{Xe}$ and $\mathrm{Cl}_{2}$}

A laser assisted collision can briefly be described as a reaction,

$$
\mathrm{A}+\mathrm{B}+\mathrm{h} v \rightarrow \mathrm{A}+\mathrm{B}^{*}
$$

where the photon energy is tuned so as to resonate with neither A or B as an unperturbed atom. Such reactions can be described as photon transitions between (AB)* molecular potentials. By changing the laser frequency, the initial conditions for a reaction can be changed and its outcome can be selectively controlled. The reaction pair then complete the second "half" of their collision on the excited potential curve.

Reactions of $\mathrm{Xe}^{*}$ with $\mathrm{Cl}_{2}$ are accurately described by an electron transfer at the seam between the valence potential surface and the ionic $\mathrm{Xe}^{+}-\mathrm{Cl}_{2}{ }^{-}$potential surface. This reaction has been called a harpoon reaction by Polyanyi. A two-photon laser assisted reactive collision on this surface can be represented as the reaction

$$
\mathrm{Xe}+\mathrm{Cl}_{2}+2 \mathrm{~h} v \rightarrow \mathrm{XeCl}^{*}+\mathrm{Cl}
$$

where the two-photon energy $2 \mathrm{~h} v$ is resonant with neither $\mathrm{Xe}$ nor $\mathrm{Cl}_{2}$ as an unperturbed species. Such reactions are described as photon transitions between transient molecular states of the collision pairs, identical to collision-induced absorption. The excited pair then complete the dissociative "half collision" on the upper molecular potential. By changing the excitation frequency, the initial conditions for the half collision can be changed and its outcome selectively controlled.

In reactions involving $\mathrm{Xe}$ with $\mathrm{Cl}_{2}$ or $\mathrm{HCl}$, the collision pairs are excited via a direct two-photon transition to the lowest $\mathrm{Xe}^{+}-\mathrm{Cl}_{2}{ }^{-}$ionic surface. A broad absorption band has been observed in this system for two-photon excitation between 290-320 nm. ${ }^{42}$ We monitor the fluorescence spectra from the product channels to study energy disposal following the first half-collision of the reagents. The different excitation frequencies sample different $\mathrm{Xe}-\mathrm{Cl}_{2}$ internuclear separations on the ionic surface. A specific example for a $\mathrm{C}_{2 \mathrm{v}}$ collision geometry is shown in Fig. 6.1. As a result the fluorescence spectra show vibrational structure that is a function of excitation wavelength. The spectra are discussed in terms of a saddle point on the ionic surface for a dissociating chlorine atom and a bound, vibrationally excited $\mathrm{XeCl}^{*}$; accordingly, a two dimensional map of energy disposal $v s$. excitation is obtained.

Castex ${ }^{43}$ recently observed spectra of $\mathrm{Xe}-\mathrm{Cl}_{2}$ dimers formed in a supersonic beam and excited by two $308 \mathrm{~nm}$ photons. She obtained a product spectrum significantly narrower than that observed by Setser. ${ }^{44)}$ In experiments presented here, we show the difference can be attributed to different initial conditions on the ionic surface, as proposed by Castex. The different spectra can be obtained from collision pairs in a gas cell.

42 J. K. Ku, G. Inoue, and D. W. Setser, J. Phys. Chem., 87, 2990 (1983).

43 M. Boivineau, J. Le Calve', m. C. Castex, and C. Jouvet, Chem. Phys. Lett. 128, 528(1986).

44 J.K. Ku, G. Inoue, and D.W. Setser, J. Phys. Chem., 87, 2990 (1983). 
From Setser 45 , we know that two-photon excitation at $308 \mathrm{~nm}$ is possible near the shallow Vander-Waals minimum at $3.4 \mathrm{~A}$ on the ground surface. This corresponds to point "A" on the ionic surface shown in Fig. 6.1. The resultant dissociation shown on Fig. 6.1 is expected to produce the $\mathrm{XeCl}^{*}$ product in a low vibrational state with a narrow spectrum. For excitation at $290 \mathrm{~nm}$, the Xe$\mathrm{Cl}_{2}$ distance must be greater, corresponding to point "B". The resultant trajectory is expected to produce vibrationally excited $\mathrm{XeCl}^{*}$. Tuning of the excitation laser to shorter wavelengths should then excite pairs on the upper surface with a higher vibrational temperature.

\subsection{Experimental Approach}

We have implemented a significant number of improvements in the laser and detectors for our most recent attempt to study laser-assisted reactive collisions of $\mathrm{Xe}$ and $\mathrm{Cl}_{2}$. We installed a new intensified diode array detector (EG\&G PARC 1420R-1024-HQ), after the demise of the previous detector (The QE of the old photocathode was severely degraded by ions sputtered from the face of the channel plate electron multiplier; the new detector provides a protective grid for the photocathode.) The replaced detector has a measured quantum efficiency of $18 \%$ at $300 \mathrm{~nm}$. The detector flat field response and the spectral response of the overall detection system (HR640 monochromator plus detector) have been measured for use in correcting spectra.

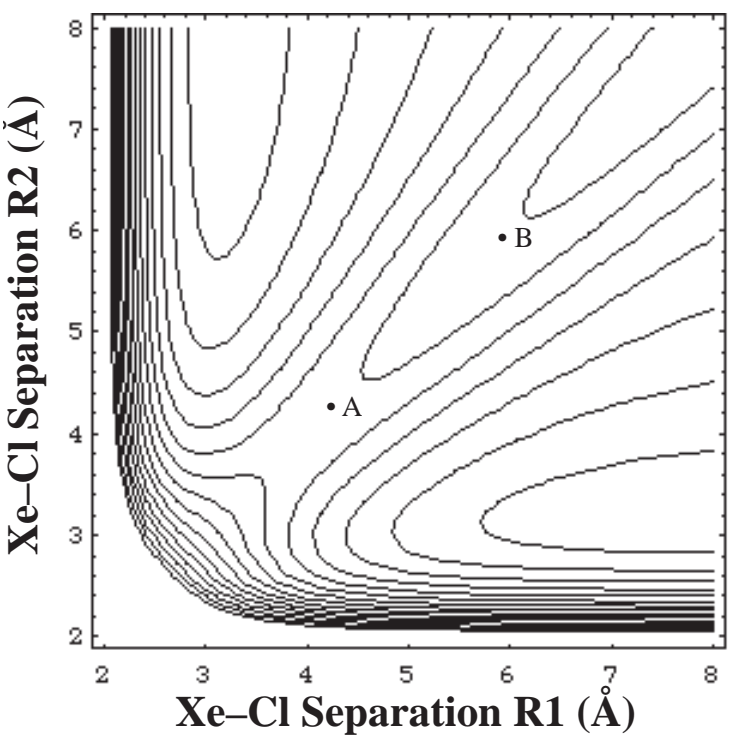

The laser performance has also been Fig. 5.1. Illustration of the reactive potential energy significantly enhanced for these experiments. We surface for the $\mathrm{Xe}^{+}-\mathrm{Cl}_{2}{ }^{-}$system. The points $\mathrm{A}$ and are using a Rhodamine dye laser consisting of a $\mathrm{B}$ represent initial conditions corresponding to two Littman oscillator followed by two amplifiers. The different excitation wavelengths.

laser beam is spatially filtered and expanded between each gain region. The power amplifier is longitudinally pumped by a Quanta-Ray model DCR-2 Nd:YAG laser. The dye laser output is frequency doubled using a BBO crystal scanned by a precision galvomotor to produce wavelengths from 280 to $320 \mathrm{~nm}$. By AR coating the beam-steering prisms and lenses, we are able to deliver $\mathrm{UV}$ powers over $7 \mathrm{~mJ} /$ pulseon target .

Care has been taken to minimize superfluorescence and ionization effects by limiting the laser energy densities at the detection region. A series of relay lenses with 1 meter focal lengths are used to transport the laser beam from the frequency doubling crystal to the sample chamber. Because we monitor fluorescence signals in the perpendicular direction to the laser beam, this experiment is not directly sensitive to superfluorescence signals. The observation region is kept upstream by approximately two Rayleigh lengths from the final laser focus in the chamber. The excitation diameter is measured to be $400 \mu \mathrm{m}$ at the point of observation. To detect possible saturation effects, then, the fluorescence signal was measured as a function of laser power for a range of focus conditions and laser intensities in order to verify that we observe the power-squared dependence characteristic of two-photon transitions and assuring that superfluorescence, photodissociation, and photoionization do not distort the data.

$45 \mathrm{Ku}$, et. al., ibid 
In order to observe the nascent vibrational distributions, we must ensure that collision rates are much less than the radiative rate for the reaction products. This implies the need to work at the lowest possible pressures ( $<3$ Torr). This constraint, of course, limits the signal from the experiment and demands long integration times and averages of many spectra to obtain acceptable signal-to-noise ratios. We have obtained spectra under these conditions, and the observed boundfree spectra illustrate the expected dependence on excitation wavelength (Fig. 6.2). The final spectra were corrected for the measured flat-field response of the detector and for the overall spectral response of the monochromator-detector system to allow quantitative analysis.

\subsubsection{Refinement of the $\mathrm{XeCl}^{*}(\mathrm{~B})$ potential}

We first compared the model spectra with the fluorescence of $\mathrm{XeCl}^{*}(\mathrm{~B})$ excited by laser assisted reactions of $\mathrm{Xe}+\mathrm{Cl}_{2}$ at xenon pressures of 400 Torr. These pressures led to rapid relaxation of the vibrational excitation and equilibrium vibrational distributions, so that a vibrational temperature can simulate the vibrational distribution. Initial modeling attempted unsuccessfully to use the

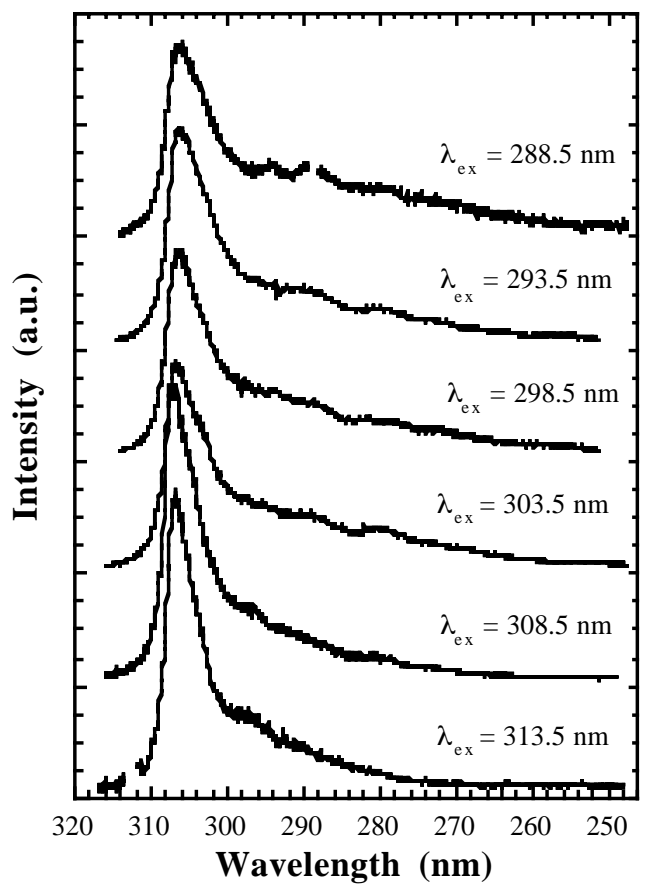

Fig. 6.2. Sample fluorescence spectra observed following two-photon excitation in $\mathrm{Xe}-\mathrm{Cl} 2$ gas mixtures for excitation wavelengths between $2885 \AA$ and $3135 \AA$ A.

ground state and excited state potentials used by Simons to model reactions of xenon metastables with $\mathrm{Cl}_{2} .{ }^{46}$ Unfortunately, these potentials were unable to even describe the well-defined main peak in our high pressure fluorescence spectrum.

46 K. Johnson, J. P. Simons, P. A. Smith, C. Washington, and A. Kvaran, Molec. Phys. 57, 255(1986). 
We obtain better comparison with our data and the data of Setser's group 47 by adjusting $\mathrm{R}_{\mathrm{e}}$ for the $\mathrm{XeCl}^{*}(\mathrm{~B})$, the steepness of the repulsive wall, and the shape of the radial dependence for the dipole moment. The Setser's spectra were obtained following quenching of atomic metastables at very low pressures. The details of these modeling efforts were described in the thesis of James Kohel, submitted to DOE in a previous progress report.

Since the completion of James' thesis, we have made efforts to model the spectra using the ground state potential determined from the $\mathrm{Xe}-\mathrm{Cl}$, beambeam scattering experiments of Aquilanti, et. al. 48 Their results were unpublished at the time of the studies by Setser and Simons. For all reasonable shapes of the excited potential consistent with Setser's experiments, we could model our

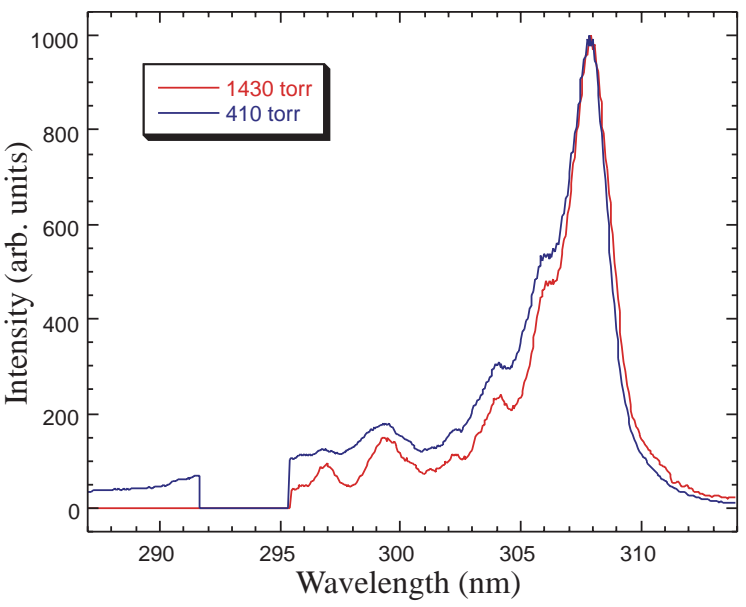

Fig. 6.3 Comparison of fluorescence at high and low pressures, demonstrating greater relaxation at higher pressures.

measurements only if we assumed very high vibrational temperatures $(900 \mathrm{~K})$ at the time of fluorescence; since the radiative lifetime is on the order of $30 \mathrm{~ns}$, we initially felt this temperature might be unreasonable at pressures of $400 \mathrm{~T}$.

In the past year, however, we measured spectra at higher pressures to record the changes in spectral lineshape. As shown in Fig. 6.3, at $1430 \mathrm{~T}$ we observed a spectrum markedly more relaxed than the one at $400 \mathrm{~T}$. In Fig. 6.4 we show a comparison of the model spectra at vibrational temperatures from 300 to $900 \mathrm{~K}$ with the experimental spectrum at $1430 \mathrm{~T}$. The higher pressure spectrum is consistent with a lower vibrational temperature at the time of fluorescence. We are now convinced that the high temperatures required to fit the data are reasonable and that this new $\mathrm{XeCl} \mathrm{B}$ potential is in agreement with high and low pressure data. A comparison of a spectral model of Setser's experiment using an identical potential is shown as Fig. 6.5.

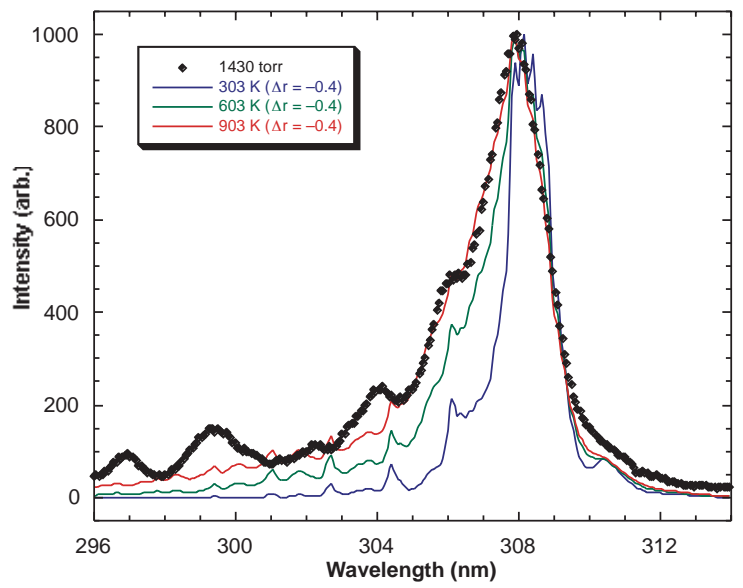

Fig. 6.4. Comparison of data with spectra models at various vibrational temperatures.

47J. H. Kolts, J. E. Velazco, and D. w. Setser, J. Chem. Phys. 71, 1247(1979).

48 V. Aquilanti, D. Cappelletti, V. Lorent, E. Luzzatti, and F. Pirani, "The ground and lowest exctied states of XeCl by atomic beam scattering," Chem. Phys. Lett. 192, 153(1992). 


\subsection{Simulation of Bound-Free Spectra}

The bound-free emission spectrum resulting from transitions between a single vibrational level $\left|v^{\prime}\right\rangle$ to all lower states $\left|\varepsilon^{\prime \prime}\right\rangle$ in the vibrational continuum is given by 49

$$
\mathrm{I}_{v}(v)=\left(64 \pi^{4} / 3 h\right) v^{3}\left|\left\langle\varepsilon^{\prime \prime}\left|\mu_{\mathrm{e}}(R)\right| \mathrm{v}^{\prime}\right\rangle\right|^{2}
$$

where $v=v_{0}+E_{v}-\varepsilon$ is the transition energy. The spectral intensity has units of photons $\sec ^{-1}$ wavenumbers $^{-1}$; in order to compare the simulated spectra to the experimental spectra recorded per unit wavelength we make use of the relationship

$$
\mathrm{I}_{v}(\lambda)=\mathrm{I}_{v}(v)(\mathrm{d} v / \mathrm{d} \lambda) \propto v^{5}\left|\left\langle\varepsilon^{\prime \prime}\left|\mu_{\mathrm{e}}(R)\right| \mathrm{v}^{\prime}\right\rangle\right|^{2}
$$

The observed spectra is the superposition of

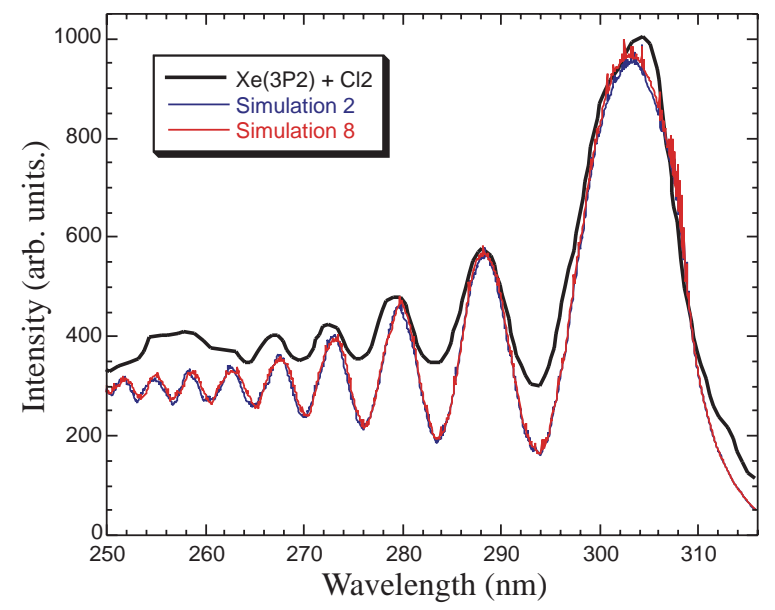

Fig. 6.5 Comparison of spectral model with Setser's experimental data (black line). The frequency and phase of the oscillations are sensitive to the location and slope of the repulsive wall of the B state potential.

transitions originating from many vibrational levels in the upper state; i.e.

$$
\mathrm{I}(\lambda)=\sum_{v=0}^{*} \mathrm{P}_{v} \mathrm{I}_{v}(\lambda)
$$

where $\mathrm{P}_{v}$ is the population in the vibrational level $v$ and $v^{*}$ is the highest energetically accessible state.

The wavefunctions $\left|v^{\prime}\right\rangle$ and $\left|\varepsilon^{\prime \prime}\right\rangle$ are solutions of the radial Schroedinger equation and were obtained numerically using the Numerov finite difference method as described by Cooley. ${ }^{50}$ The free wavefunctions were energy normalized by matching the amplitude at large $R$ to the asymptotic solution. Only the portion of this wavefunction in the Franck-Condon overlap region with upper bound state was stored. Once the wavefunctions have been obtained, the above Franck-Condon integral is evaluated for the dipole-allowed transitions $\Delta J=J^{\prime}-J^{\prime \prime}=0, \pm 1$ and over a range of continuum energies $\varepsilon^{\prime \prime}$. The emission wavelength is a function of this continuum energy:

$$
\lambda=1 /\left(T_{e}+G_{v^{\prime}}-\varepsilon^{\prime \prime}\right),
$$

where $G_{v^{\prime}}$ is the vibrational energy in the upper bound state and $T_{e}$ is the minimum energy of the upper potential relative to that of the ground state potential.

We note the possibility of bound-bound transitions in the $\mathrm{XeCl}(\mathrm{B}-\mathrm{X})$ system. Calculations indicate the existence of 3-4 bound states in the shallow (approx. $16 \mathrm{~cm}^{-1}$ ) $\mathrm{XeCl}(\mathrm{X})$ potential. These weakly bound states however, are not supported in the effective potentials for $J$ values much greater than 10-20, and we expect the relative contribution to the overall spectra from transitions to these lower bound states to be very slight given the likely population of high $J$ rovibrational levels

49 K. Tamagake and D. W. Setser, J. Chem. Phys. 67, 4370 (1977).

50 J. W. Cooley, ibid; see also J. Cashion, "Testing of diatomic potential-energy functions by numerical methods," J. Chem. Phys. 39, 1872 (1963). 
in the laser assisted reaction. We therefore neglect bound-bound transitions in our simulations. This approach is further justified by noting the absence of any discrete features in the observed spectra.

As noted, we anticipate substantial population of high rotational levels, particularly for lower vibrational levels where a large fraction of the reaction exoergicity remains available for rotational and translational excitation of $\mathrm{XeCl}^{*}$. The effect of a distribution of rotational levels is to smooth out the high frequency oscillations and to smear out the sharp cutoff at the blue end of the emission spectrum which are shown in Fig. 6.6. The calculation of individual spectra from this large number of rovibrational levels involves considerable computational expense, however. We have obtained satisfactory results by approximating the rotational distribution using only selected rotational levels. The highest vibrational levels, in fact, were represented by single rotational levels since in the observed wavelength region there was little difference between the spectra from the energetically allowed rotational levels.

In order to accurately model the observed fluorescence spectra, we must include contributions from vibrational levels as high as $v \approx$ 160 and rotational levels as high as $J \approx 600$. The task of representing an observed spectra with this large number of basis states can, in many cases, be greatly simplified by employing the statistical approach of surprisal analysis.

\subsection{Surprisal Analysis}

An analysis of reactive collisions typically seeks to characterize the energy disposal in the reaction process in terms of its specificity, where a high specificity corresponds to narrow product distributions, and a low specificity corresponds to more random or chaotic outcomes. Surprisal analysis is an information-theoretic approach which can be used to provide a compact measure of this specificity in energy disposal. The method of surprisal analysis consists in seeking that distribution of minimum information content (i.e., maximum entropy) that is consistent with the determined constraints; ${ }^{51}$ and, as such, is a useful technique for interpreting detailed data from gasphase reactions involving far-from-equilibrium distributions. We are now in the process of fitting various forms of surprisal, which define the vibrational distributions and hence the spectral lineshapes, to the experimental spectra.

51 R. D. Levine and J. L. Kinsey, "Information-theoretic approach: Application to molecular collisions," in Atom--Molecule Collision Theory: A Guide for the Experimentalist, R. B. Bernstein, ed. (Plenum Press, New York, 1979), pp. 693750. 


\subsection{A Simple Repulsive Release Model}

In the following we describe a simple repulsive release model to predict rotational and vibrational excitation in the laser-assisted reactive collision. The current model is related to previous "retreat-coordinate models," 52 in particular the DIPR (direct interaction with product repulsion) model formulated by Polanyi. ${ }^{53}$ The DIPR model emphasizes the repulsive interaction in reactions of type $\mathrm{A}+\mathrm{BC} \rightarrow \mathrm{AB}+\mathrm{C}$, where the dissociation of the $\mathrm{B}-\mathrm{C}$ bond results in a rapid energy release directed along this bond axis. The current model also includes a possible attractive interaction as the product A-B bond is "switched on" between the extended nuclei.

\section{General considerations}

The DIPR model describes a "direct" reaction where the reaction coordinate is monotonically increasing following the reactive encounter and secondary encounters are excluded. 54 The repulsive and attractive interactions are further described as occurring in a stepwise or sequential fashion; i.e., B.C repulsion precedes $\mathrm{A}-\mathrm{B}$ attraction. The interactions are also considered separable: and no long range interaction exists between $\mathrm{A}$ and $\mathrm{BC}$ during $\mathrm{B} \cdot \mathrm{C}$ repulsion and no interaction exists between $\mathrm{AB}$ and $\mathrm{C}$ during $\mathrm{A}-\mathrm{B}$ attraction. ${ }^{55}$ To the extent that the repulsive energy release occurs rapidly at large $\mathrm{Xe}-\mathrm{Cl}_{2}$ collision distances and is a significant fraction of the total reaction exoergicity, the laser-assisted reaction is well described by this idealized interaction model.

In the reaction $\mathrm{A}+\mathrm{BC} \rightarrow \mathrm{AB}+\mathrm{C}$, both reagent orbital angular momentum and product repulsion may, in general, contribute to rotational excitation of reaction products. ${ }^{56}$ The first contribution is found to be dominant for reactions in which both the attacking atom $\mathrm{A}$ and its reaction partner $\mathrm{B}$ are heavy (e.g., $m_{\mathrm{A}}, m_{\mathrm{B}} \approx 80 \mathrm{amu}$ ), while the atom $\mathrm{C}$ ejected as a product is light $(\approx 1 \mathrm{amu})$. The second contribution is most prominent where (a) the repulsive energy release $\mathfrak{R}$ is large, (b) the reaction proceeds through a bent intermediate configuration, and (c) the mass of the ejected atom is significant relative to that of the atom $\mathrm{B}\left(m_{\mathrm{C}} \geq m_{\mathrm{B}}\right)$. In this case, release of the $\mathrm{B} \cdot \mathrm{C}$ repulsion imparts substantial recoil velocity to $\mathrm{B}$ and, in this bent configuration, this velocity largely results in rotation in the $\mathrm{AB}$ product.

52 J. C. Polanyi and J. L. Schreiber, "The dynamics of bimolecular reactions," in Kinetics of Gas Reactions, vol. 6A of Physical Chemistry --- An Advanced Treatise, H. Eyring, W. Jost, D. Henderson, eds. (Academic Press, New York, 1974).

53 P. J. Kuntz, M. H. Mok, and J. C. Polanyi, “'Distribution of reaction products. V. Reactions forming an ionic bond, M + XC (3D)," J. Chem. Phys. 50, 4623 (1969).

54 D. G. Truhlar and D. A. Dixon, "Direct-mode chemical reactions II: Classical theories," in Atom-Molecule Collision Theory: A Guide for the Experimentalist, R. B. Bernstein, ed. (Plenum Press, New York, 1979), pp. 595--653.

55 M. G. Prisant, C. T. Rettner, and R. N. Zare, “A direct interaction model for chemiluminescent reactions," J. Chem. Phys. 81, 2699 (1984).

56 N. H. Hijazi and J. C. Polanyi, "Magnitude and orientation of rotation in exchange reactions A + BC $\rightarrow$ AB + C*," J. Chem. Phys. 63, 2249 (1975). 


\section{Model details}

In the laser-assisted reaction, the repulsive energy release follows immediately the photon induced transition to the reactive ionic surface, and thus the internuclear separations at which this release occurs are that of the original collision pair. The repulsive energy release is initiated by the electron jump event whereby the $\mathrm{BC}$ reagent crosses from the initial covalent potential to the repulsive ionic potential. The magnitude of energy released is the energy liberated by removing one $\mathrm{Cl}$ atom from the initial internuclear separation on the repulsive potential to a distance for which the neutral $\mathrm{Cl}$ atom no longer acts on the internal energy of the $\mathrm{Xe}^{+}-\mathrm{Cl}_{2}{ }^{-}$. By the time the $\mathrm{Cl}$ reaches the equilibium separation of the $\mathrm{Cl}_{2}{ }^{-}$potential, $\mathrm{Xe}^{+}$ and $\mathrm{Cl}_{2}^{-}$move toward the $\mathrm{XeCl}^{*} \mathrm{~B}$ equilibrium

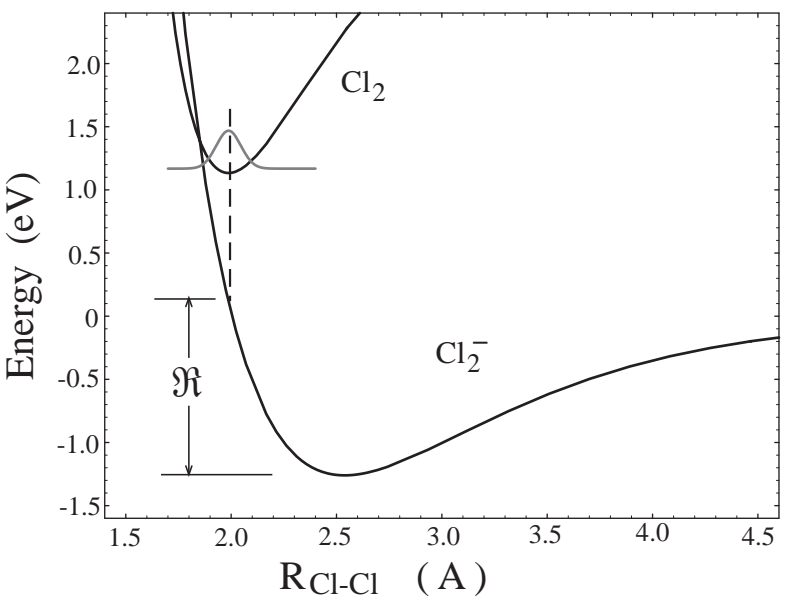

Fig. 6.7. Potential energy curves showing the repulsive release at the equilibrium separation for neutral chlorine following two-photon excitation to the ion surface.

separation and rotate, thus modifying the attractive field experienced by the $\mathrm{Cl}$ atom. As well this attraction acts on average on the center of mass motion of the $\mathrm{XeCl}^{*}$ molecule rather than on the relative motion of the two atoms. Thus the Repulsive release is better expressed by

$$
\Re=\mathrm{EA}(\mathrm{Cl})-D_{\mathrm{e}}\left(\mathrm{Cl}_{2}\right)-\mathrm{EA}_{\mathrm{v}}\left(\mathrm{Cl}_{2} ; r\right)-\mathrm{D}_{\mathrm{e}}\left(\mathrm{Cl}_{2}{ }^{-}\right) \sim 1.1 \mathrm{eV},
$$

as shown in Fig. 6.7 (Here, $\mathrm{EA}_{\mathrm{v}}\left(\mathrm{Cl}_{2} ; r\right.$ ) is the vertical electron affinity for $\mathrm{Cl}_{2}$ at internuclear separation $r, D_{\mathrm{e}}\left(\mathrm{Cl}_{2}\right)$ the dissociation energy, and $\mathrm{EA}(\mathrm{Cl})$ the electron affinity of the chlorine atom.) , rather than the repulse energy for motion of the $\mathrm{Cl}$ atom to infinity along the $\mathrm{Cl}_{2}$ bond,

$$
\Re=\mathrm{V}\left(\mathrm{Cl}_{2}^{-} ; r\right)-\mathrm{V}\left(\mathrm{Cl}_{2}^{-} ; \mathrm{r} \rightarrow \infty\right) \text {. }
$$

This latter case gives $\mathfrak{R} \sim 0.2 \mathrm{eV}$, which produces calculated spectra in large disagreement with the experimental ones.

This repulsion is directed along the initial $\mathrm{Cl}-\mathrm{Cl}$ internuclear axis, and the momentum from this repulsive impulse is shared equally between the two $\mathrm{Cl}$ atoms. These atoms then experience a change in velocity of

$$
\Delta v=\sqrt{ } \Re / m_{\mathrm{Cl}} .
$$

The velocity of the $\mathrm{Cl}^{-}$ion after this repulsive interaction is given by $\mathbf{v}(\mathrm{B})=\mathbf{v}_{0}(\mathrm{~B})+\Delta v \hat{\mathbf{r}}_{\mathrm{B}}$, where $\mathbf{v}_{0}$ is the initial velocity of this ion and the direction vector $\hat{\mathbf{r}}_{\mathrm{B}}$ is the unit vector pointing outward along the former $\mathrm{Cl}-\mathrm{Cl}$ bond axis toward this negative ion. The relative velocity of the Xe and $\mathrm{Cl}$ atoms is then

$$
\mathbf{v}=\mathbf{v}(\mathrm{B})-\mathbf{v}(\mathrm{A})=\mathbf{v}_{0}(\mathrm{~B})+\Delta v \hat{\mathbf{r}}_{\mathrm{B}}-\mathbf{v}_{0}(\mathrm{~A}) .
$$

For thermal velocity distributions, the initial velocities $\mathbf{v}_{0}$ are small relative to $\Delta v$, so that we approximate the final velocity by the second term. The relative velocity $\mathbf{v}$ results in a rotational angular momentum in the $\mathrm{XeCl}$ product

$$
\mathbf{L}=\mu \mathbf{R} \times \mathbf{v},
$$


where $\mathbf{R}$ is the vector directed from $\mathrm{Xe}^{+}$to $\mathrm{Cl}^{-}$and $\mu$ is the reduced mass for the $\mathrm{XeCl}$ diatom. The magnitude of this angular momentum is given by

$$
L=\mu R v \sin \alpha=\mu \sqrt{ } \Re / m_{\mathrm{Cl}} \sin \alpha
$$

where $\alpha$ is the angle between the $\mathrm{Cl}-\mathrm{Cl}$ and $\mathrm{Xe}-\mathrm{Cl}$ internuclear axes (see Fig. 6.8). From the magnitude $L$ we define an angular momentum quantum number $J$ such that

$$
J \approx L / h \text {. }
$$

The component of linear momentum directed along the $\mathrm{Xe}-\mathrm{Cl}$ internuclear axis is released into both the translational (centerof-mass motion) and the vibrational (relative motion) coordinates of the $\mathrm{XeCl}$ product. The component of $\mathfrak{R}$ contributing to vibrational excitation is

$$
1 / 2 \mu(\mathbf{v} \cdot \hat{\mathbf{R}})^{2}=\left(\mu / m_{\mathrm{Cl}}\right) \mathfrak{R} \cos ^{2} \alpha
$$

We may note that, in general, reactions of this type, as $m_{\mathrm{A}}$ is decreasing relative to $\mu_{\mathrm{AB}}$, a larger fraction of the repulsive energy component $\mathfrak{R} \cos ^{2} \alpha$ is directed into vibration and a lesser fraction enters center-of-mass motion in AB.

Immediately following the rapid repulsive interaction an attractive interaction which brings $\mathrm{Xe}^{+}$and $\mathrm{Cl}^{-}$together. The energy released in this attractive interaction is defined by conservation of energy, i.e.

$$
\boldsymbol{A}=Q-\mathfrak{R}-T_{\mathrm{e}},
$$

where $Q$ is the total exoergicity of the reaction for a given excitation wavelength (defined as in Section 5.1) and $T_{\mathrm{e}}$ is energy of the minimum of the $\mathrm{XeCl}(B)$ potential relative to the minimum of the ground state potential. The energy liberated in this attractive interaction is released entirely into internal energy in the $\mathrm{XeCl}$ product. Not all of this energy, however, results in vibrational excitation; a portion of this energy must go into overcoming the angular momentum barrier as the atoms are drawn together.

The final internal energy of the diatom product after the repulsive and attractive interactions is thus given by

$$
E_{\text {int }}=1 / 2 \mu v^{2}+A=\left(\mu / m_{\mathrm{Cl}}\right) \mathfrak{R} \cos ^{2} \alpha+\boldsymbol{A} .
$$

Angular momentum is conserved during the attractive interaction. The rotational energy of the final product is then

$$
E_{\mathrm{rot}}=L^{2} / 2 \mu r_{\mathrm{eq}}=1 / 2\left(\mu / m_{\mathrm{Cl}}\right)\left(r^{2} / r_{\mathrm{eq}}^{2}\right) \Re \sin ^{2} \alpha
$$

where $r$ is the initial $\mathrm{Xe}-\mathrm{Cl}$ separation and $r_{\text {eq }}$ is the equilibrium bond length on the effective potential for a given $J$ level. The vibrational energy is determined from conservation of energy:

$$
E_{\mathrm{vib}}=E_{\mathrm{int}}-E_{\mathrm{rot}}=1 / 2\left(\mu / m_{\mathrm{Cl}}\right)\left(1-\left(r^{2} / r_{\mathrm{eq}}{ }^{2}\right) \sin ^{2} \alpha\right) \Re \cos ^{2} \alpha+\boldsymbol{A} \text {. }
$$


The repulsion was noted to be dependent on the bond length of the $\mathrm{Cl}_{2}$ reagent at the time of excitation. This dependence is incorporated in the "distributed as in photodissociation" (DIP) description ${ }^{57}$ of the electron jump mechanism leading to dissociation. In the DIPR-DIP model recoil energy is determined by a direct mapping of the bond extension in the initial covalent state onto the lower ionic potential, i.e.,

$$
\mathfrak{R}\left(r_{\mathrm{Cl}_{2}}\right)=\mathfrak{R}_{0}-(G / d)\left(r_{\mathrm{Cl}_{2}}-r_{\mathrm{e}}\right),
$$

where $-G / d$ is the slope of the repulsive potential which is assumed to be approximately constant over the region of the transition, and $\mathfrak{R}_{0}$ is the mean value of the repulsion defined as in Eq. (5.8) for internuclear separation equal to $r_{\mathrm{e}}$, the equilibrium bond length in the upper state. This mapping is illustrated in Figure 5.4. Higher repulsive energies, it can be seen, result from transitions at smaller bond extensions $r_{\mathrm{Cl}_{2}}$ while transitions at greater bond extensions result in lower repulsive energies. The magnitude of the slope of the repulsive potential determines how strongly repulsion varies with this bond length.

The distribution of vibrational phases in the upper state can be taken as the square of the ground state vibrational wavefunction

$$
\mathrm{P}\left(r_{\mathrm{Cl}_{2}}\right) \propto \exp \left[-\left(r_{\mathrm{Cl}_{2}}-r_{\mathrm{e}}\right)^{2} / d^{2}\right]
$$

where $d=\sqrt{\mathrm{h}} / c w_{\mathrm{e}} \mu_{\mathrm{Cl} 2}$ is the displacement from equilibrium bond length $r_{\mathrm{e}}$ to the classical turning points. The distribution of repulsive energies can then be expressed as

$$
\mathrm{P}(\Re) \propto \exp \left[-\left(\Re-\Re_{0}\right)^{2} / G^{2}\right],
$$

where $G$ is a measure of the width of the repulsion given by minus $d$ times the repulsive potential slope.

The DIP interpretation may be incorporated into the description of reaction geometries in laser-assisted reactive collisions. The laser-induced transition occurs at internuclear separations $r_{\mathrm{XeCl}_{2}}=r_{\mathrm{x}}$ and $r_{\mathrm{Cl}_{2}}$ such that

$$
\Delta E=2 \mathrm{hc} / \lambda=\mathrm{V}\left(\mathrm{Xe}^{+}+\mathrm{Cl}_{2}^{-} ; r_{\mathrm{XeCl}_{2}}, r_{\mathrm{Cl}_{2}}\right)-\mathrm{V}\left(\mathrm{Xe}+\mathrm{Cl}_{2} ; r_{\mathrm{XeCl}_{2}}\right) \text {, }
$$

where $2 \mathrm{hc} / \lambda$ is the two photon excitation energy, $\mathrm{V}\left(\mathrm{Xe}+\mathrm{Cl}_{2}\right.$; $)$ the van der Waals potential that mediates the collision process, and $\mathrm{V}\left(\mathrm{Xe}^{+}+\mathrm{Cl}_{2}^{-}\right)$the reactive ion-pair potential. The ionic potential is represented as

$$
\mathrm{V}\left(\mathrm{Xe}^{+}+\mathrm{Cl}_{2}^{-} ; r_{\mathrm{XeCl}_{2}}, r_{\mathrm{Cl}_{2}}\right)=\mathrm{IP}(\mathrm{Xe})-\mathrm{EA}_{\mathrm{v}}\left(\mathrm{Cl}_{2} ; r_{\mathrm{Cl}_{2}}\right)-\mathrm{e}^{2} / r_{\mathrm{XeCl}_{2}},
$$

where $\mathrm{IP}(\mathrm{Xe})$ is the ionization potential for xenon and $\mathrm{EA}\left(\mathrm{Cl}_{2} ; r_{\mathrm{Cl}_{2}}\right)$ is the vertical electron affinity for $\mathrm{Cl}_{2}$ at internuclear separation $r \mathrm{Cl}_{2}$. The variation of the vertical electron affinity with respect to $r$ $\mathrm{Cl}_{2}$ near $r_{\mathrm{e}}$ as may be approximated as ${ }^{58}$

57 D. R. Herschbach, Faraday Discuss. Chem. Soc. 55, 233 (1973).

58 D. R. Herschbach, ibid. 


$$
\mathrm{EA}_{\mathrm{v}}\left(\mathrm{Cl}_{2} ; r_{\mathrm{Cl}_{2}}\right)=\mathrm{EA}_{\mathrm{v}}\left(\mathrm{Cl}_{2} ; r_{\mathrm{e}}\right)+(G / d)\left(r_{\mathrm{Cl}_{2}}-r_{\mathrm{e}}\right)
$$

where $-G / d$ is the slope of the slope of the $\mathrm{Cl}_{2}{ }^{-}$potential at $r_{\mathrm{e}}$. We use Eqs. (5.22)-(5.24) to obtain the characteristic separation $r_{\mathrm{x}}$ at which the laser-induced transition occurs:

$$
r_{\mathrm{x}}=\mathrm{e}^{2} /\left[\mathrm{IP}(\mathrm{Xe})-\mathrm{EA}_{\mathrm{v}}\left(\mathrm{Cl}_{2} ; r_{\mathrm{e}}\right)+(G / d)\left(r_{\mathrm{Cl}_{2}}-r_{\mathrm{e}}\right)-2 \mathrm{hc} / \lambda\right]
$$

As a final comment, we also note that in the above we have assumed no interaction with the $\mathrm{Xe}$ atom during the rapid repulsive energy release. This assumption is valid in so far as the characteristic time $\tau$ for energy release is less than the vibrational period of the $\mathrm{XeCl}$ diatom:

$$
\tau \ll T_{\mathrm{vib}}=(2 \pi / \mathrm{c}) / \omega_{\mathrm{e}}
$$

For the $\operatorname{XeCl}(B)$ state, the value $\omega_{\mathrm{e}}=194.235 \mathrm{~cm}^{-1}$ gives $T_{\mathrm{vib}} \approx 10^{-12} \mathrm{~s}$. The interaction time for this repulsion may now be estimated as follows: The average force $F_{0}=G / d$ in this linear approximation of the repulsive potential results in a constant acceleration $a=F_{0} / \mu_{\mathrm{Cl} 2}$ until a bond extension $r^{\prime}$ is reached such that $r^{\prime}-r_{\mathrm{e}}=(d / G) \Re_{0}$. The interaction time $\tau$ (assuming a transition at $r=r_{\mathrm{e}}$ with zero initial relative velocity) is given by

$$
\tau=\sqrt{ } 2\left(\mathrm{r}^{\prime}-\mathrm{r}_{\mathrm{e}}\right) / \mathrm{a}=(\mathrm{d} / \mathrm{G}) \sqrt{ } 2 \mu_{\mathrm{Cl} 2} \Re_{0}
$$

From the above analysis we obtain a crude estimate of $\approx 7.5 \mathrm{fs}$ for the interaction time $\tau$. Hence the condition (5.26) for separability is indeed satisfied.

\section{Calculational details}

The DIPR-DIP model as described above was implemented numerically. The program calculates the quasiclassical trajectories on the reactive surface defined by the repulsive and attractive interactions, with initial conditions sampled using Monte Carlo techniques. ${ }^{59}$ Initial values of the coordinates in each collision were determined by a fixed excitation wavelength and randomly selected values of orientation angle and vibrational phase (or, equivalently, repulsive energy) in the reactant molecule. Trajectory outcomes were sorted according to the resultant angular momenta $J$ and vibrational energies $E_{\mathrm{vib}}$.

We have assumed an isotropic weighting of reagent orientations; i.e., $P(\theta)$ constant, for $-\pi / 2<$ $\theta \leq \pi / 2$ where $\theta$ is the angle between $\mathrm{Cl}-\mathrm{Cl}$ and $\mathrm{Xe}-\mathrm{Cl}_{2}$ bonds. $\theta=0$ corresponds to a collinear approach geometry, while $\theta= \pm \pi / 2$ corresponds to a broadside $\left(C_{2 \mathrm{v}}\right)$ geometry. We exclude angles greater than $\pi / 2$ and less than $-\pi / 2$ since we assume the electron jump process which precedes formation of the $\mathrm{Xe}-\mathrm{Cl}$ bond occurs via the nearest $\mathrm{Cl}$ atom.

The $\mathrm{Xe}-\mathrm{Cl}_{2}$ initial separation is the crossing radius $r_{\mathrm{x}}$ in Eq. (6.26). The initial $\mathrm{Xe}^{+}-\mathrm{Cl}^{-}$ internuclear separation $R_{\text {init }}$ can by expressed in terms of the above:

$$
R_{\text {init }}=\left(r_{\mathrm{x}}^{2}+r_{\mathrm{Cl}}^{2}-2 r_{\mathrm{Cl}} r_{\mathrm{x}} \cos \theta\right)^{1 / 2},
$$

59 I. M. Sobol', A Primer for the Monte Carlo Method, (CRC Press, Boca Raton, FL 1994). 
where $r_{\mathrm{Cl}}=r_{\mathrm{Cl}_{2}} / 2$, and $\theta$ is again the angle defined by the axis between the Xe atomic center and the $\mathrm{Cl}_{2}$ center of mass and the axis between the $\mathrm{Cl}$ nuclei . The angle $\alpha$ between the $\mathrm{Cl}_{2}$ internuclear axis and the product $\mathrm{XeCl}$ bond axis is given by $\alpha=\pi-\beta$ (see Fig. 6.8), where $\beta$ is determined from

$$
\beta=\cos ^{-1}\left[\left(R_{\text {init }}^{2}+r_{\mathrm{Cl}}^{2}-r_{\mathrm{x}}^{2}\right) /\left(2 R_{\text {init }} r_{\mathrm{Cl}}\right)\right] .
$$

The product angular momentum $J$ is calculated as a function of the angle $\alpha$ using Eqs. (6.12) and (6.13). The final vibrational energy is calculated as in Eq. (6.18). Trajectories resulting in negative vibrational energies are considered nonreactive and are discarded. Approximately 5-25\%, depending on excitation wavelength, of the trajectories fail in this manner. A greater number of trajectories are unsuccessful for longer excitation wavelengths as the exoergicity is not large enough to include the larger values of repulsive energy.

For the remaining successful trajectories, we identify a vibrational quantum number $v$ with this classical energy such that $E_{\mathrm{vib}} \approx E_{v}$, where $E_{v}$ is the eigenenergy calculated by a numerical integration of the Schroedinger equation using the full effective potential (see Section 6.3.1). The classical correspondence principle employed here is valid given a sufficient density of quantum states.

The program was run on an IBM RS/6000 model $58 \mathrm{H}$ with up to $10^{8}$ trajectories to average over initial conditions as described above. Values for the orientation angle $\theta$ and repulsion $\Re$ were randomly selected, and each trajectory was weighted by the joint probability $P(\Re) \cdot P(\theta)$. Trajectory outcomes were sorted according to the resulting angular momentum $J$ and $v$ to give normalized product state populations.

The DIPR model predicts the nascent vibrational and rotational distribution for two-photon excitation of the xenon and chlorine collision pair. This nascent distribution function is shown in Fig. 6.9 for a laser wavelength of $314 \mathrm{~nm}$.

For each vibrational level, the bound-free spectra of the $\mathrm{XeCl}^{*}$ molecule is averaged over a selected set of rotational states representing the nascent distribution. The final fluorescence spectrum is then generated by summing spectra for each vibrational transition using the nascent vibrational distribution. An example calculated spectrum is compared with the excperiment in Fig. 5.7 for a laser wavelength of $313.5 \mathrm{~nm}$. Though not perfect, the comparison between the model and experiment is quite remarkable. Preliminary calculations show reasonable agreement at higher reaction energies and shorter excitation wavelengths with the experimental spectra shown in Fig. 5.2 , including the oscillations on the blue wing and the dip at $300 \mathrm{~nm}$. Calculations for larger rotational and vibrational quanta are required for a full comparison. 


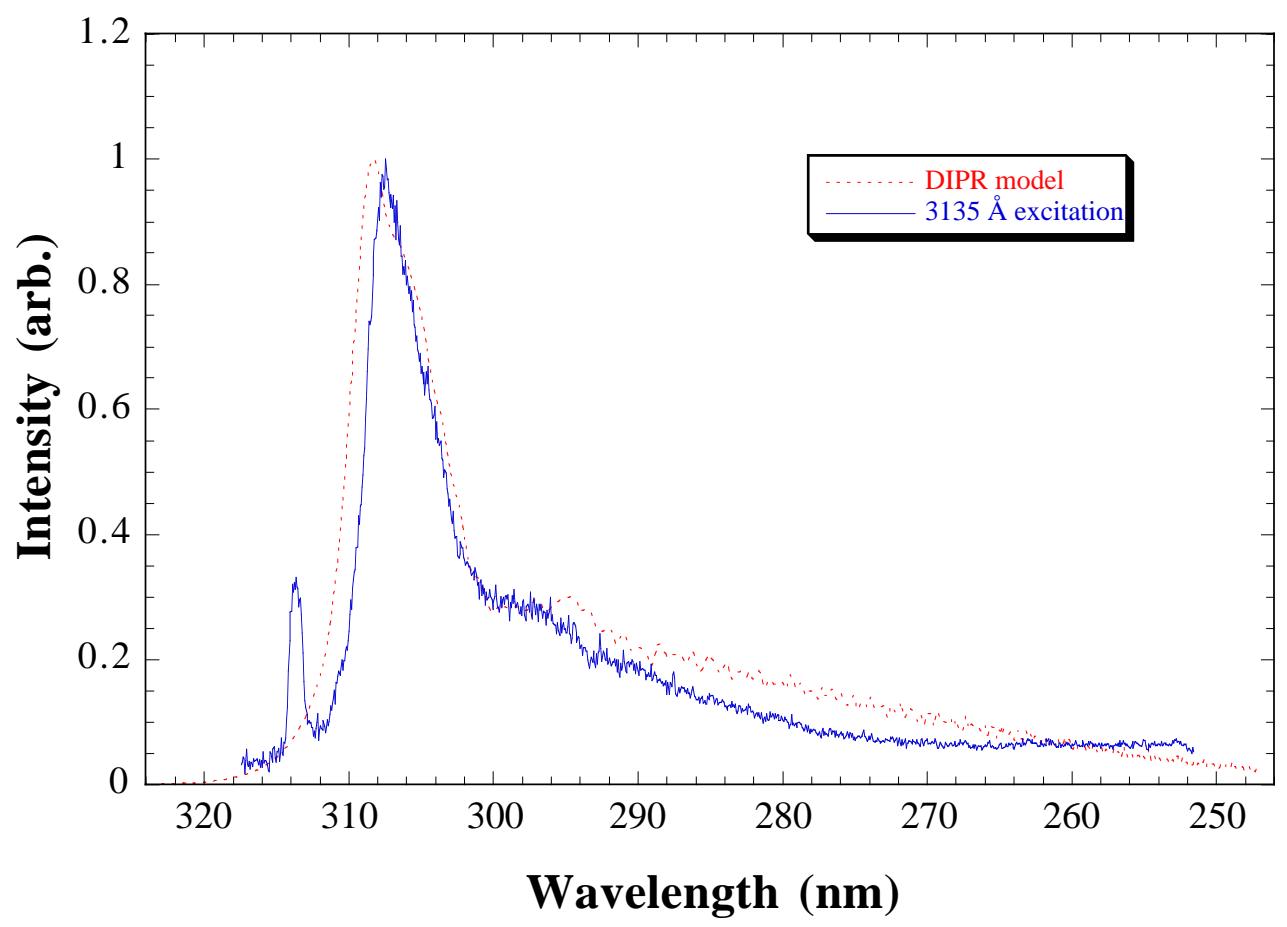

Fig. 6.9. Comparison of measured spectrum and theoretical spectrum which results from the vibrational and rotational distributions calculated with the DIPR model and shown in Fig. 6.6. 


\subsection{Coherent Raman Spectroscopy of Ions and Radicals in Discharges}

An outstanding problems in high pressures plasmas is the understanding of the reactions of ions and radicals. At pressures near one atmosphere, new ions in the form of clusters are formed and reactions of all forms of ions are complicated by the possibility of termolecular reactions. ${ }^{60,61}$ An understanding of the reactions of radicals and molecular ions in high density discharges has been impeded by the lack of a suitable diagnostic. Mass spectrometers are inoperable at high pressures and sampling of cluster ions through a differentially pumped orifice produces a supersonic expansion causing the size of the cluster to grow. Spontaneous Raman spectroscopy and infrared adsorption are hampered by the low particle densities and high background luminosities inherent in these environments. Coherent Raman spectroscopy is not so hampered and also offers the advantages of high spectral and time resolution in measuring spectra. Using a wavelength meter developed in our laboratory for pulsed lasers we can measure the frequency of Raman resonances of discharge species to a precision of a few parts in $10^{8}$. [Pub. 12] We also achieved an order of magnitude improvement in the sensitivity of coherent Raman spectroscopy by using the Raman induced Kerr effect (RIKE). We obtained spectra with sufficient sensitivity to obtain the first coherent Raman spectra of molecular ions in the vapor phase and are poised to make the first Raman spectra of molecular ions.

\subsection{Recent progress for obtaining OHD-RIKES of discharges}

We significantly improved a RIKES spectrometer by improving the optical design so as to remove much of the birefringence and background. These improvements are discussed in sections 7.1.1 and 7.1.2. Improvements in the operation of the high-pressure discharge, installation of a thyratron and electronics enabled the synchronization of a high pressure discharge and spectroscopy lasers. A description of this pulsed discharge is given in section 7.1.3. Manuscripts describing the OHD-RIKES spectrometer [Pub. 11] and the precisely tunable, narrow-band dye laser [Pub. 8] it uses were published. We also studied saturation of the Raman pump process and demonstrated that RIKES saturates at orders of magnituded higher laser intensities than CARS [Pub. 13]. While the discharge was being completed and tested we looked for radicals in a low pressure ( 10 Torr) linear glow discharge. We were unsuccessful in observing the $\mathrm{N}_{3}$ radical, but we have made the first measurements of the Raman spectra of the $\mathrm{O}_{2}{ }^{1} \Delta$ metastable molecule. We have also observed a very strange behavior of the $\mathrm{CO}_{2}$ molecule in discharges. These experiments are described in section 2.2.

\subsubsection{Improvements in S/N with RIKES}

We have made improvements in the apparatus to reduce the background and to optimize the signal. Craig McCluskey, a new student at the time, reduce the birefringence by nearly and order of magnitude and then noticed systematic oscillations in the remaining background shown in Fig. 7.1. We attributed these to either an etalon effect or to oscillations in the birefringence of the sample cell windows.

60 Chien-Yu Kuo and J.W. Keto, "Dissociative Recombination of Electrons in Electron Beam Excited Argon and High Densities," J. Chem. Phys. 78, 1851-1860 (1983).

${ }^{61}$ D. R. Bates and W. Lowell Morgan, Phys. Rev. Lett. 64, 2258(1990). 
No previous graduate student reported seeing these oscillations, but looking back at their data, we saw weak oscillations obscured by noise. To obtain any advantage from our reduction in birefringence, we needed to eliminate these oscillations. With careful diagnostics, we finally determined that the oscillations were interferences generated in the sample cell windows. Multiple reflections in the windows generated weak beams $(0.16 \%)$ at the photodetector which had twice the residual birefringence as the light directly transmitted through the window. Because of this greater birefringence the analyzing polarizer made the reflected beams nearly equal in amplitude with the directly transmitted beam at the photodetector. As the wavelength of the probe laser was scanned, these

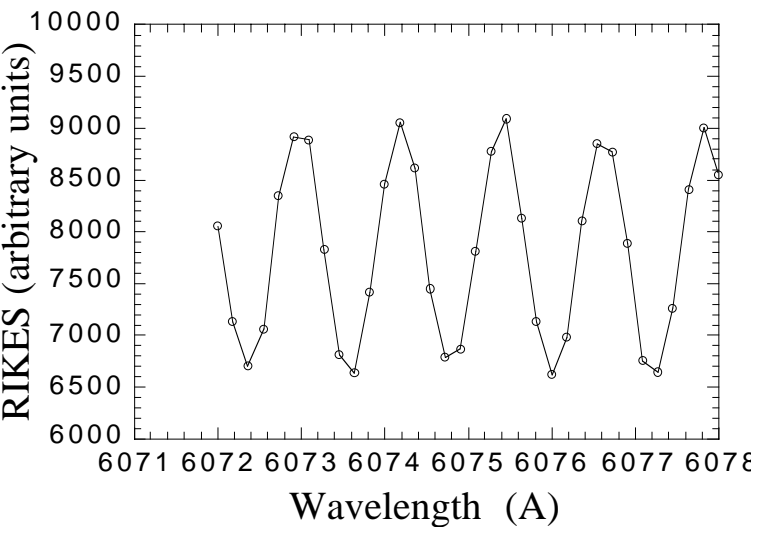
beams generated interference fringes.

We solved the problem by using windows with 30 minutes of wedge angle. These caused sufficient divergence between the reflected and transmitted beams so that both did not hit the detector. The resulting background, shown in Fig. 7.2 , corresponds to an extinction of $10^{8}$ which is state-of-the-art for polarization spectroscopy. In Fig. 7.3 we compare a Raman spectra of $\mathrm{N}_{2}$ at 20 Torr, obtained with the wedged windows, with a previous spectrum. I should point out that the wedged windows make the apparatus very difficult to align; and nearly impossible to use stress to reduce the birefringence further. (Stress nulls the birefringence for only one glass thickness. Since

Fig. 7.1 Oscillations in background as a function of probe laser wavelength. with the pump laser off.

the thickness varies across the window, we can null the birefringence only for a line across the beam.)

We had hoped to make the apparatus simpler to align by using optics made of Shott SF57 glass. This glass has a static birefringence a factor of 100 smaller than BK7 glass and would have greatly simplified alignment. We have now determined by experiment that the damage threshold for SF57 glass is too low for use in our spectrometer, and the backgrounds of Figs. 7.2 and 7.3 are the best that we can obtain.

\subsubsection{Heterodyne experiments}

In previous experiments we used heterodyne techniques at the detector. In this technique a small local oscillator field $\sqrt{ } \mathrm{I}\left(\omega_{1}\right)$, where $\mathrm{I}\left(\omega_{1}\right)$, is the probe laser intensity, is mixed with the Raman signal on the photodetector. The response of the detector is then

$$
\mathrm{I}_{\mathrm{sig}}\left(\omega_{1}\right)=\gamma \mathrm{I}\left(\omega_{1}\right)+2 \sqrt{ } \alpha_{\mathrm{R}} \gamma \mathrm{I}\left(\omega_{1}\right) \mathrm{I}\left(\omega_{2}\right)+\alpha_{\mathrm{R}} \mathrm{I}\left(\omega_{1}\right) \mathrm{I}^{2}\left(\omega_{2}\right),
$$

If the local oscillator field is made to be greater than the Raman field, then the cross term (second term in Eq. 7.1) is larger than the RIKE (third) term. Since the Raman field scales linearly with the 
density of target molecules, the cross term decreases linearly with pressure while the RIKE (or similarly CARS) signal decreases as the square of the decreasing pressure.

In the first discharge experiments we wanted to use a broad band dye laser to quickly obtain survey spectra. With a narrow band laser many millions of spectral samples must be made to continuously cover a large spectral region. OHD-RIKES has been done broadband with a narrow band probe and broadband pump. We mistakenly assumed our case (broadband probe, narrowband pump) would be symmetrically similar, but there is an important distinction.

In our experiments with both methane and hydrogen which used broadband pump and probe lasers, we verified that we were operating in OHD (optically heterodyne detection) mode by observing that the signals scaled linearly in pressure and pump intensity. For recent experiments with $\mathrm{N}_{2}$ (similar to Fig. 7.3) using a broadband probe laser and narrowband pump laser, we measured a signal which scales as the pressure squared. We also observed that increasing the local oscillator intensity by uncrossing the polarizer gave no increase in signal! The local oscillator field and the Raman field are apparently not sufficiently coherent to generate a significant cross term in Eq. 7.1 We believed that this is due to using a narrow band pump laser with a broad band probe laser. The narrow band pump generates a narrowband Raman field at the detector which is coherent with only the fraction $\Gamma_{\text {Raman }} / \Gamma_{\text {laser }}$ of the probe bandwidth.

To obtain sufficient intensity in the heterodyne field when generated from a broadband dye laser, the incoherent part of the probe intensity generates very large shot noise. When one uses a broadband pump laser the incoherent part of the Raman signal

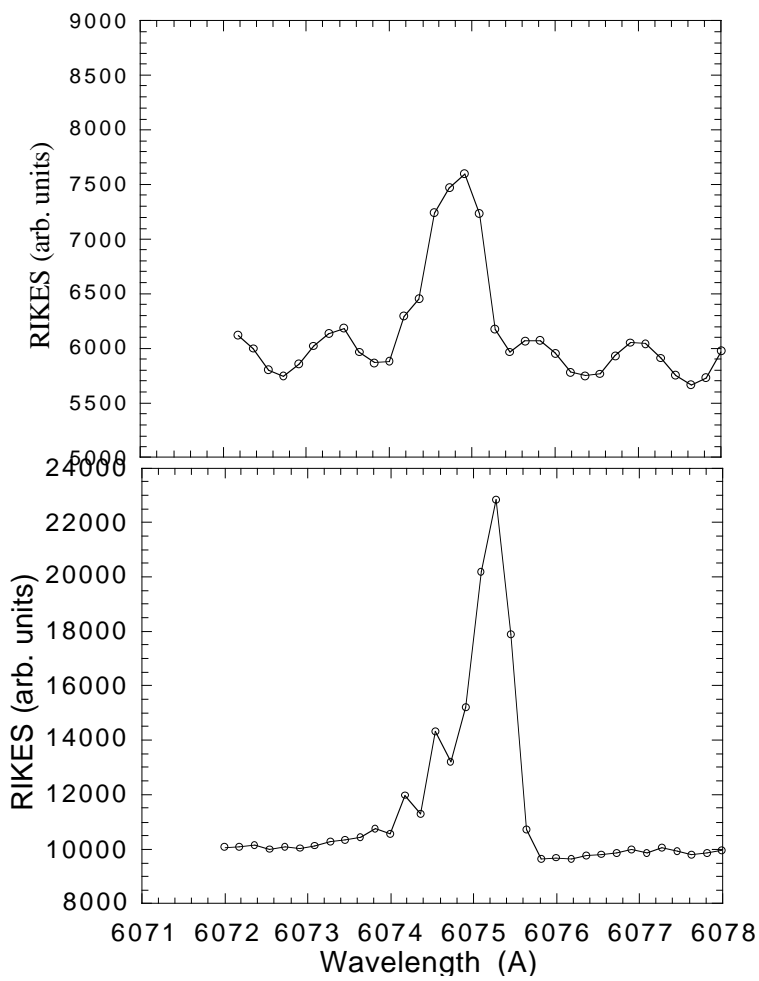
Torr, without wedged windows. Bottom panel: is very weak and the contributed noise is negligible. Spectra at 5 Torr with recent improvements. This For these reasons we could not use OHD-RIKE for spectra has a S/N of 400 for 50 laser shots at a survey scans and began using CARS instead. Raman gain 16x smaller than for the top panel. OHD-RIKE will be used for high resolution spectroscopy.

The use of CARS required improving the detection of the anti-Stokes light. We added a photomultiplier (PMT) as a detector and the combination of a Bragg notch filter to reject the pump laser and a small double monochromator to reject scattered dye laser light. This detector combined with our charge integrated, photon counting technique gives CARS spectra with high sensitivity and very little noise. Noise from scattered laser light is less than one count in 100 laser shots. Dark noise in the PMT contributes less than 1 count in $2 \times 10^{5}$ laser shots. 


\subsubsection{Improvements in the discharge circuitry, electrode geometry, and synchronization electronics}

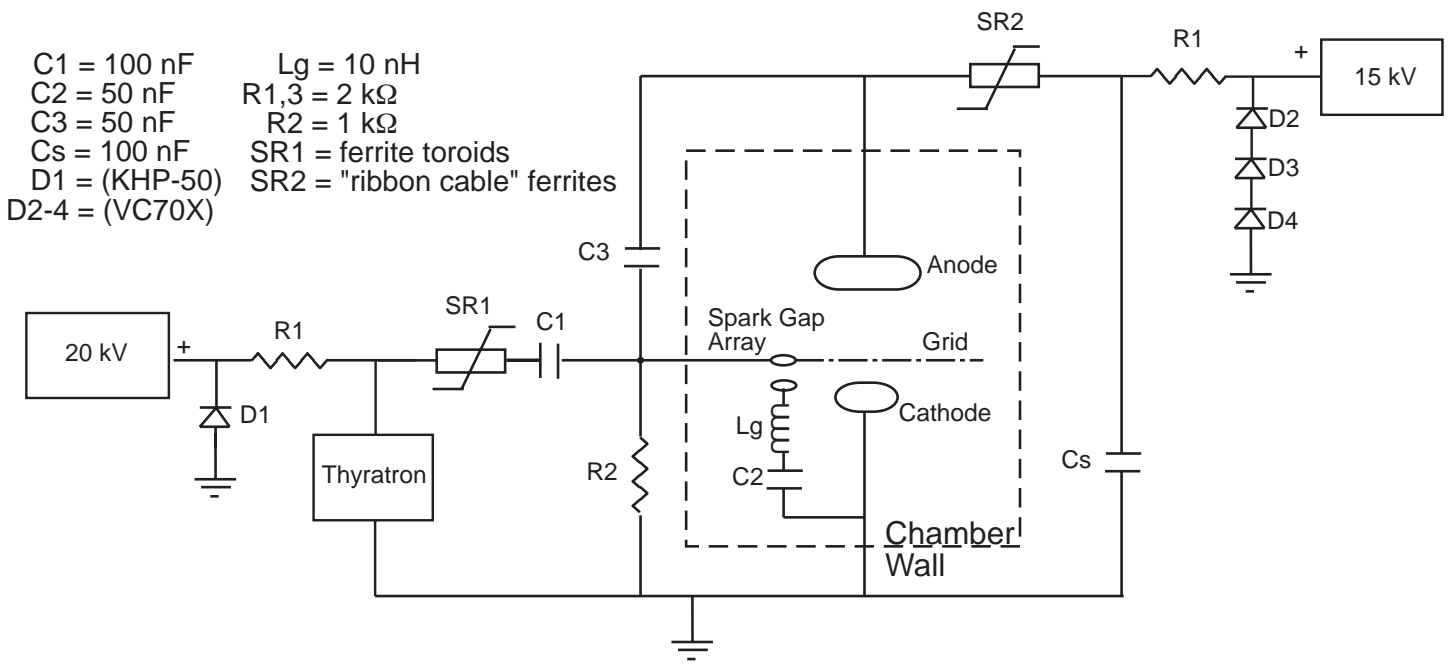

Fig.7. 4 Schematic of the discharge circuit.

The basic circuit for the high pressure discharge is shown in Fig. 7.4 Similar to an excimer laser, the circuit uses a $\mathrm{C}-\mathrm{C}$ charge transfer principle to rapidly charge the main discharge capacitors. Charging first begins by the charging of $\mathrm{C} 1$ and the sustainer capacitor Cs by two high-frequency switching power supplies. Over the ms times required for this charging the resistor $\mathrm{R} 2$ grounds $\mathrm{C} 2$ and $\mathrm{C} 3$. Hence the sustainer supply precharges $\mathrm{C} 3$ to $\sim 8 \mathrm{kV}$ as well as charging the sustainer bank. When the capacitors are charged, the thyratron is triggered with the correct time delays relative to the YAG laser so that the laser will be synchronized relative to the discharge. Note that both the discharge and YAG laser output have significant time delays from their respective triggers but the time jitters have been measured to be on the order of 2 ns.

The thyratron acts to short the charged side of $\mathrm{C} 1$ causing the other side to rise to a large negative voltage. This begins the charging of $\mathrm{C} 2$ following the breakdown of the spark-gap array as well as additional charging of the anode capacitor C3. During this additional charging of $\mathrm{C} 3$, the saturable

magnet SR2 acts as a diode preventing this charge Fig. 7.5 Discharge electrodes including C2. from moving to the sustainer capacitor. The spark gap array consists of 100 individual inductors and spark gaps spaced evenly on each side of the

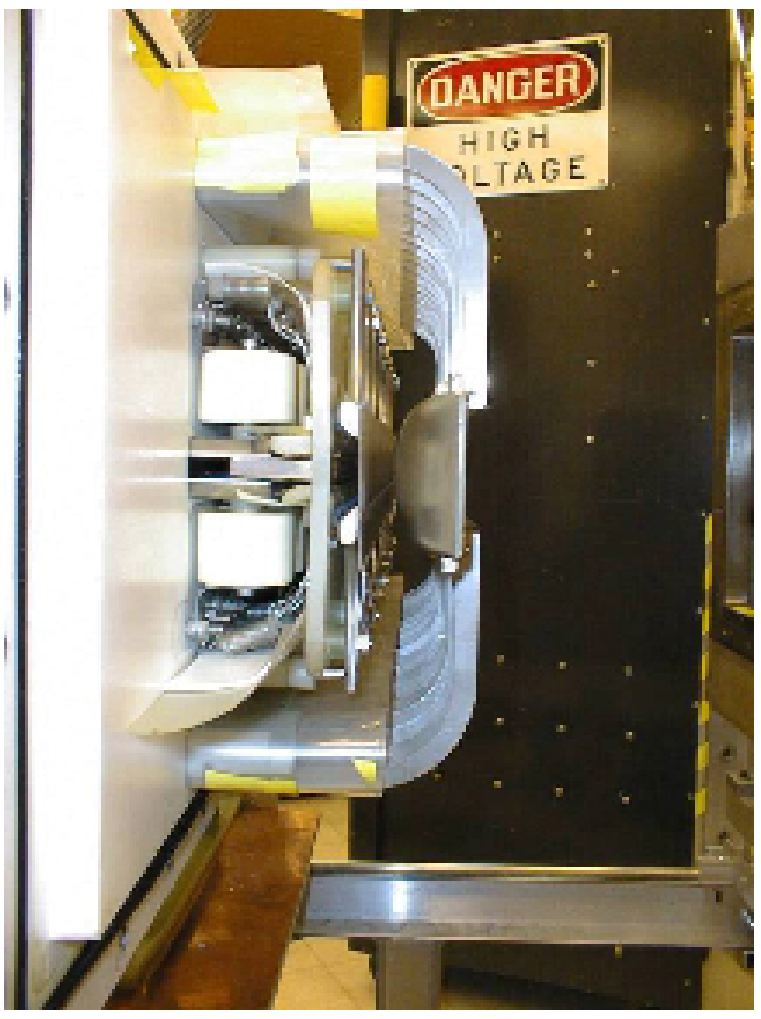

Anode is on right, grid is just to left. cathode and used to UV preionize the cathode-togrid volume. When the voltage across $\mathrm{C} 2$ is sufficient, the grid-cathode gap breaks down and a glow discharge is formed. Since the grid is formed of a stainless steel mesh, the cathode discharge 
acts to uniformly preionize the anode-to-grid volume. When the anode-grid volume impedance falls to a sufficiently smaller value, C3 discharges. The anode electrode, shown in profile in Fig. 2.5 , is cast in a Rogowski profile of graphite composite and nickel plated to a thickness of $2 \mathrm{~mm}$.

In the first prototype of the high pressure discharge circuit, we had difficulties getting sufficient charge on the anode capacitor C3. We determined that the inductive loop for $\mathrm{C} 3$ was more than a factor of 5 larger than for $\mathrm{C} 2$, hence most of the charge from $\mathrm{C} 1$ was transferred to $\mathrm{C} 2$. When the grid-cathode discharge fires the remaining charge from $\mathrm{C} 1$ flows to the discharge and the anode would never reach a sufficient value to break down the grid-anode gap. During the first year of the contract period an undergraduate, Jeremy Highley, redesigned the geometry of the capacitor banks in order to equate the charging inductances for $\mathrm{C} 2$ and $\mathrm{C} 3$. He then manufactured and tested the new layout. At the same time an all stainless steel gas box for the discharge was designed and manufactured. A picture of the new capacitor geometry and gas box, which also employs a thyratron rather than a spark gap, is shown in Fig. 7.6. Over the past contract year we finished the installation of the thyratron and optical fibers for the electronics used to synchronize the YAG laser and the discharge. The discharge is now fully operational and synchronized with the YAG laser.

Measurements of the voltage across $\mathrm{C} 3$ shown in Fig. 7.7 give a risetime consistent with the new circuit inductance. In the pulse shown, the gridcathode distance and gas pressure resulted in a breakdown at approximately $8 \mathrm{kV}$, limiting the charging of $\mathrm{C} 3$ to about $15 \mathrm{kV}$. Higher pressures or a larger anode-grid spacing would result in charging nearer the capacity of the power supplies and increase the stored energy. At the voltages shown the energy deposited by $\mathrm{C} 3$ is about $6 \mathrm{~J}$ in a volume of $\sim 200 \mathrm{~cm}^{3}$. The discharge currently gives a very uniform glow at pressures of 4 atm. The time profile of emitted light from a helium discharge is shown in Fig. 7.8. The discharge as driven by $\mathrm{C} 3$ has a full width of $\sim 50 \mathrm{~ns}$. The heating of the discharge by the sustainer is also observed. The intensity for the sustained part of the discharge can be increased both by increasing the voltage and the sustainer capacitance. We currently have mounted only a small number of capacitors in the sustainer for testing.

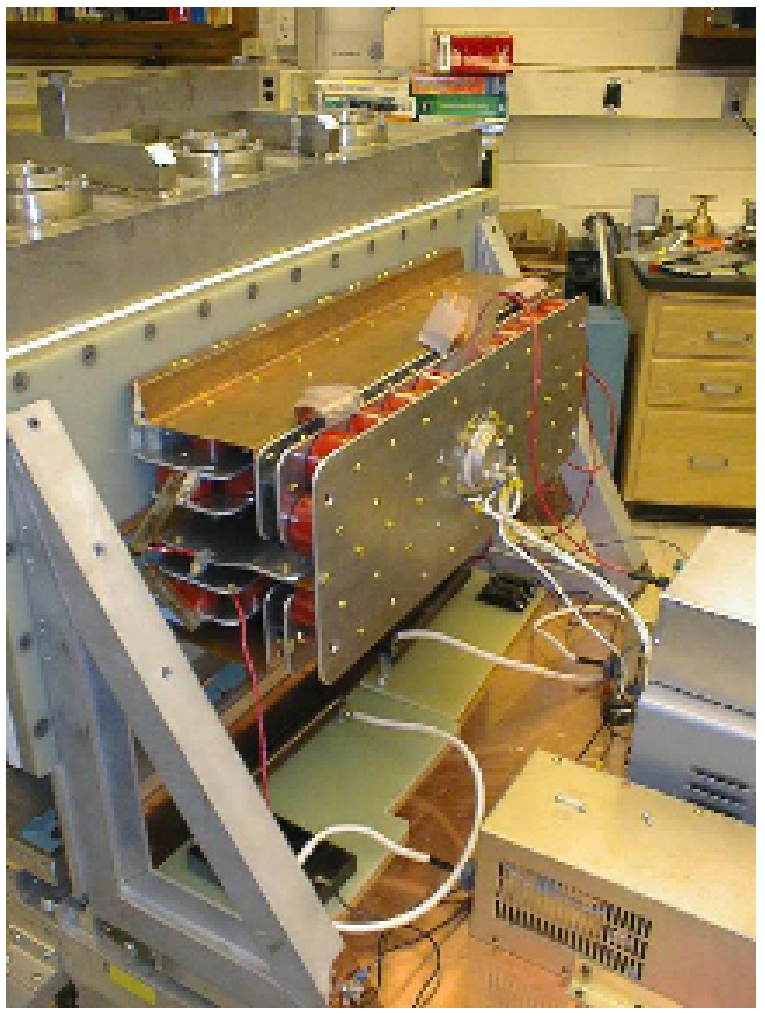

Fig. 7.6 Rear view of the discharge showing the new stainless gas box and capacitor arrangement. One can see the new thyratron, mounted on the ground electrode of the sustainer capacitor Cs, near the center of the photo. The charging and synchronization electronics are seen in the right of the picture.

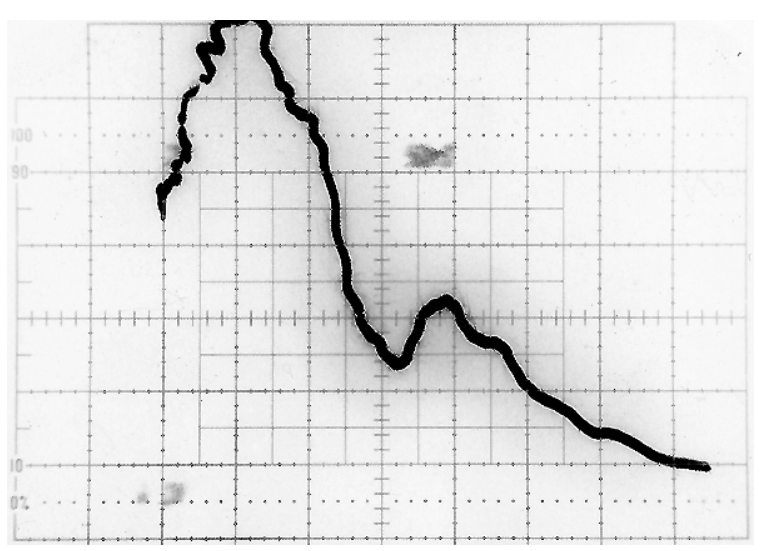

Fig. 7.7. Oscilloscope tracing of charging of $\mathrm{C}_{3}$ at $2 \mathrm{kV} / \mathrm{cm}$ and $50 \mathrm{~ns} / \mathrm{cm}$. 


\subsection{Experiments in a low pressure discharge}

These experiments were performed in the positive column of a linear glow discharge. The discharge is water cooled with hollow, cylindrical electrodes with $2 \mathrm{~cm}$ diameter. The discharge tube decreases after the electrodes to a diameter of $5 \mathrm{~mm}$ for a length of $50 \mathrm{~cm}$. The lasers enter through a window, through the center of the electrode, and are focused to a waist at the center of the discharge. The discharge is maintained through a ballast resistor by a $10 \mathrm{kV}, 1$ amp DC power supply. Discharges are ignited at a lower pressure and maintained as the pressure is increased up to about 10 Torr. Discharge currents can be varied up to about $200 \mathrm{~mA}$.

\subsubsection{Attempts to observe the $\mathrm{N}_{3}$ radical}

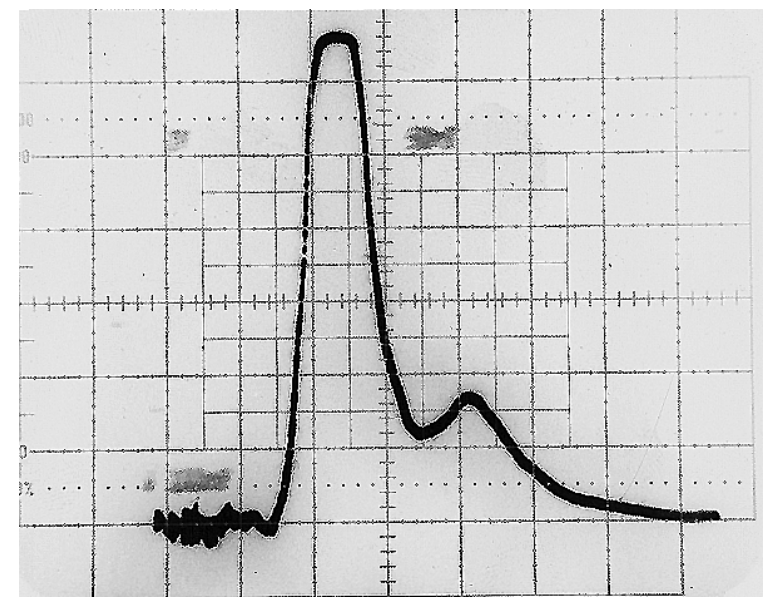

Fig. 7.8. Light output from a helium discharge displayed on a $50 \mathrm{~ns} / \mathrm{cm}$ time scale. The secondpeak results from the sustainer.

We used lower pressure targets of $\mathrm{N}_{2}$ to make improvements in the RIKE spectrometer as discussed in section 7.1.2. We then ran discharges in $\mathrm{N}_{2}$ and immediately observed an order of magnitude decrease in the intensity of the Raman spectrum in both the RIKE and CARS experiments. Some decrease was observed which could be retrieved by re-adjusting the overlap between the pump and probe beam while the discharge was running. We speculated that the Bessel profile of the discharge acts as a lens thus disturbing the overlap of the two laser beams. Even with re-optimization we observed a signal an order of magnitude smaller. In analogy with research in oxygen discharges, we expected $\mathrm{N}_{2}$ dissociation by electron impact. We hoped that this might lead to the formation of $\mathrm{N}_{3}$ by association,

$$
\begin{aligned}
& \mathrm{N}_{2}+\mathrm{e} \rightarrow 2 \mathrm{~N}+\mathrm{e} \\
& 2 \mathrm{~N}_{2}+\mathrm{N} \rightarrow \mathrm{N}_{3}+\mathrm{N}_{2} .
\end{aligned}
$$

The $\mathrm{N}_{3}$ molecule was previously detected after generation by the $\mathrm{F}+\mathrm{HN}_{3}$ reaction by laser induced fluorescence in the transition $\widetilde{A}{ }^{2} \Sigma_{\mathrm{u}}+(000) \leftarrow \widetilde{\mathrm{X}}^{2} \Pi_{\mathrm{g}}(000)$ at $271.96 \mathrm{~nm}$. and $\widetilde{\mathrm{A}}{ }^{2} \Sigma_{\mathrm{u}}+(010)$ $\leftarrow \widetilde{\mathrm{X}}^{2} \Pi_{\mathrm{g}}(010)$ at $270.86 \mathrm{~nm} .{ }^{62}$ Weak fluorescence features were tentatively assigned to $\tilde{\mathrm{X}}(100)$ and $X(020)$ giving approximate vibrational values $\omega_{1} " \sim 1320 \mathrm{~cm}^{-1}$ and $\omega_{2} " \sim 457 \mathrm{~cm}^{-1}$. RIKE spectroscopy would provide precise measurements of the vibrational frequencies (to $5 \times 10^{-3} \mathrm{~cm}^{-1}$ ). A very different measurement of the vibrational frequency was observed by Tian, et. al. in matrix isolated $\mathrm{N}_{3}$ by Fourier transform IR absorption. Theoretical calculations give an energy of 1324 $\mathrm{cm}^{-1}$, nearer to Setser's result. We also hoped confirm a previous rotational analysis. ${ }^{63}$ An asymmetric stretch energy v3 $=1644.68 \mathrm{~cm}^{-1}$ was measured by R. Pahnke et al.

62 R. A. Beaman, T. Nelson, D. S. Richards, and D. W. Setser, J. Phys. Chem. 91, 6090(1987).

63 A. E. Douglas and W. J. Jones, Can. J. Phys. 43, 2216(1965). 
Fig. 7.7 shows a CAR spectrum in our discharge in the vicinity of $1320 \mathrm{~cm}^{-1}$. Unfortunately we observe a CAR signal at this frequency in the windows of the discharge cell. This results either from the bulk of the glass (note that the beams have large diameters in the windows and the resulting signals are expected to be very small) or impurities on the surfaces. We could minimize this signal by moving the windows relative to the lasers. The small hump near 1320 $\mathrm{cm}^{-1}$ in Fig. 7.7 is a result of the windows. It's intensity does not vary with the gas pressure in the target cell, but it is a CAR signal, e.g. it goes away when either the pump or probe laser is blocked, and it is peaked in the blue. We can observe no Raman signal from $\mathrm{N}_{3}$, either because the radical density is too low or because the Raman intensity is weak. We will attempt this experiment again in the high pressure discharge.

\subsubsection{The $\mathrm{O}_{2}$ hotband and $\mathrm{O}_{2}{ }^{1} \Delta$}

Shown in Fig. 7.8 is a CARS spectrum of $\mathrm{O}_{2}$ in a discharge at room temperature. Spectra were obtained to search for the $\mathrm{O}_{2}{ }^{1} \Delta$ metastable state in a discharge. ${ }^{64}$ In the spectral region from 1500$1535 \mathrm{~cm}^{-1}$ we see many lines with spacing $\sim 2.0$ $\mathrm{cm}^{-1}$. Among these lines are the $\mathrm{J}^{\prime \prime}=7$ and $\mathrm{J}^{\prime \prime}=9$ $\mathrm{O}$ branch lines of $\mathrm{O}_{2}$ (Note because of the spin 0 nuclei and spin of the ground electronic state all even $\mathrm{J}^{\prime \prime}$ are missing). In the discharge $\mathrm{O}(7)$ is significantly reduced in intensity relative to $\mathrm{O}(9)$; both are reduced in intensity absolutely. This results from rotational heating by the discharge.

The closely spaced lines are $\mathrm{Q}$ branch lines of the 2-1 hotband transition of $\mathrm{O}_{2}$. The position of the lines in the spectrum are labeled appropriately. In panel $b$, measured with the discharge off, the hotband is much less intense because of the smaller vibrational temperature of the molecule. It is tempting to assign the feature at 1532 in the spectrum to the $\mathrm{O}(5)$ line, but the known position is at $1530.3 \mathrm{~cm}^{-1}$; while the 2-1 Q branch hotband should begin at 1332.71, in agreement with the bright feature in both panels. J" from 1 to 9 lie between 1531.3 and $1532.7 \mathrm{~cm}^{-1}$. Given the current broad band resolution of $0.2 \mathrm{~cm}^{-1}$, these

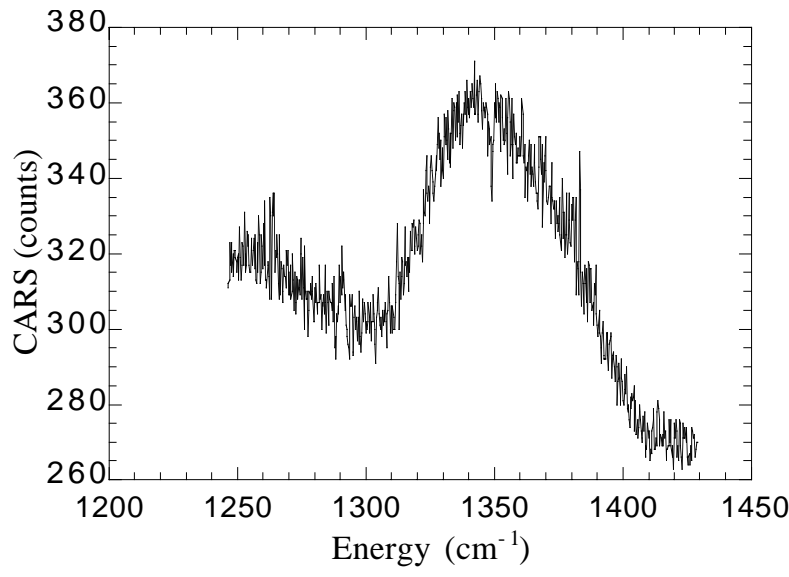

Fig. 7.7. CAR spectrum in vicinity of expected $\mathrm{N}_{3}$ resonance. Discharge current of $200 \mathrm{~mA}$ and $\mathrm{N}_{2}$ pressure of 8 Torr.

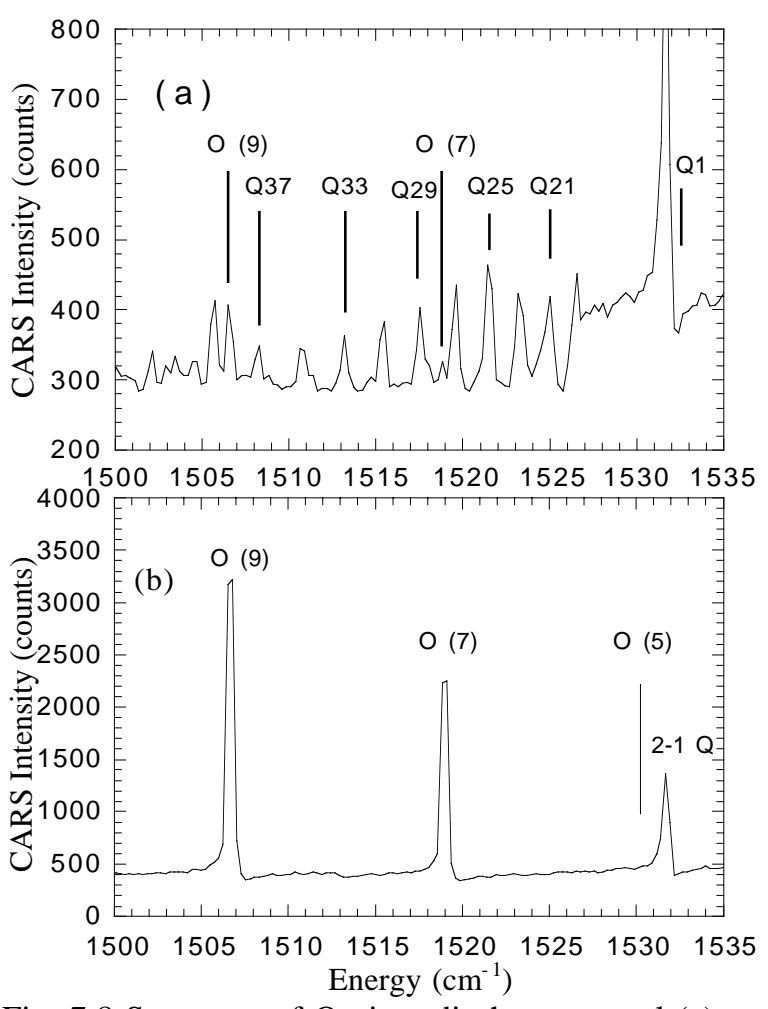

Fig. 7.8 Spectrum of $\mathrm{O}_{2}$ in a discharge, panel (a) at 10 Torr and $180 \mathrm{~mA}$ discharge current. The $\mathrm{O}$ branch lines belong to 1-0 while the Q branch lines belong to $2-1$. The panel (b) shows a room temperature spectrum with the discharge off. sum to form a broad, bright band head. In panel (a) we resolve none of the lines between $\mathrm{J}^{\prime \prime}=19$ and the bandhead. This broadening may result from mixing between the $\mathrm{v}=0, \mathrm{~J}=5$ and the $\mathrm{v}=1$,

${ }^{64}$ Jacek Borysow suggested possibility for this experiment in a private communication . 
$J "=9-17$ states; from the laser resolution alone one might expect to resolve the $v=1, J "=17$ state. This broadening may also be enhanced by electron collisions in the discharge.

Though there is no doubt of our observation of the hotband in $\mathrm{O}_{2}$, the line positions are not in good agreement with the molecular constants describing the ground state molecule. In Table I we record the $\mathrm{Q}$ branch transitions measured from the spectrum with calculations using the ground state parameters taken from Huber et al. using the rotational energy,

$$
F_{V}(J)=B_{V} J(J+1)-D_{V} J^{2}(J+1)^{2}
$$

We obtained an accurate comparison with the Q and $\mathrm{O}$ branch lines of (1-0) with the same molecular parameters up to $\mathrm{J} "=25$, both with and without the discharge. One can get an extimate from the data of Table I for the parameter (not measured to date) $\gamma_{e}=-0.00028_{3}$, where

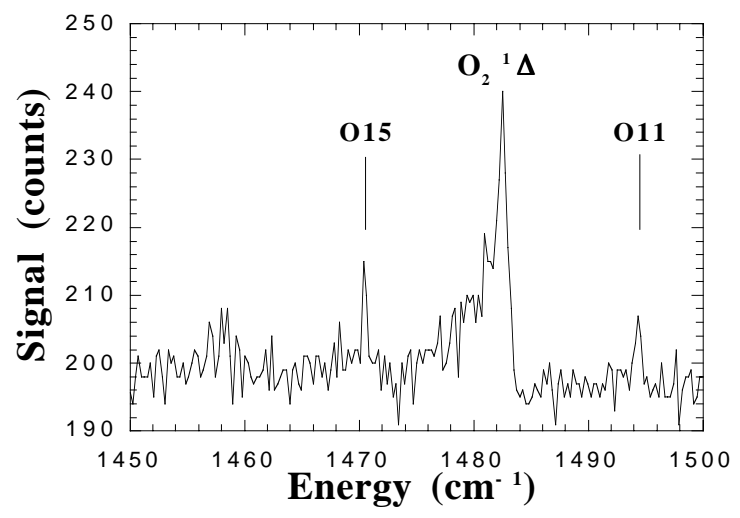

Fig. 2.9. Spectrum taken in a $100 \mathrm{~mA}$ discharge at $\sim 80 \mathrm{~K}$

$$
B_{v}=B_{e}-\alpha_{e}(v+1 / 2)+\gamma_{e}(v+1 / 2)^{2}
$$

Finally, in we show in Fig. 2.9 a scan over the region 1450-1500 cm-1 in a discharge in cold $(\sim 80 \mathrm{~K}) \mathrm{O}_{2}$. We believe the feature near 1483 is the $\mathrm{Q}$ branch spectrum of $\mathrm{O}_{2}{ }^{1} \Delta$ which should have $\mathrm{Q}(1)$ at 1483.5 .

Table I: (2-1)Q(J") line positions compared with theory.

\begin{tabular}{lcc}
\hline J" & Experiment $\left(\mathrm{cm}^{-1}\right)$ & Huber \\
\hline \hline 1 & & 1532.82 \\
3 & & 1532.66 \\
5 & & 1532.37 \\
7 & & 1531.96 \\
9 & & 1531.42 \\
11 & & 1530.75 \\
13 & & 1529.96 \\
15 & & 1529.03 \\
17 & 1527.1 & 1527.99 \\
19 & 1525.7 & 1526.81 \\
21 & 1523.8 & 1525.51 \\
23 & 1522.0 & 1524.07 \\
25 & 1520.2 & 1522.52 \\
27 & 1518.1 & 1520.83 \\
29 & 1516.0 & 1519.02 \\
31 & 1513.9 & 1517.08 \\
33 & 1511.2 & 1515.01 \\
35 & 1508.8 & 1512.82 \\
37 & 1506.3 & 1510.5 \\
39 & & 1508.05 \\
\hline
\end{tabular}

a) Molecular constants taken from K. P. Huber and G. Herzberg, "Molecular spectra and Molecular Structure, Vol. 4, Constants of Diatomic Molecules," 498-499, Van Nostrand-Reinhold, 1979. 


\subsection{3 $\mathrm{CO}_{2}$ in a discharge: quantum interference in $\mathrm{V}$-T relaxation}

Two strong Q branch transitions are observed in $\mathrm{CO}_{2}$ in the range $1200-1500 \mathrm{~cm}^{-1}$, one at 1285 $\mathrm{cm}^{-1}$ and the other at $1388 \mathrm{~cm}^{-1}$. These transitions form the famous Fermi resonance between the first overtone of bend, (020), and the symmetric stretch (100). 65 Because the energies of these normal modes are degenerate and of the same symmetry in $\mathrm{CO}_{2}$, they are strongly coupled by anharmonic and coriolis perturbations. The perturbation splits the states to their observed energies $\left(\mathrm{H}_{12} \sim 51.5 \mathrm{~cm}^{-1}\right)$, and mixes nearly equal contributions of the harmonic modes in the observed states. Then the expected wavefunctions $\psi_{\mathrm{u}}, \psi_{\mathrm{l}}$ for the upper and lower states can be written

$$
\begin{aligned}
& \psi_{\mathrm{u}}=\cos \theta|020>+\sin \theta| 100> \\
& \left.\psi_{\mathrm{l}}=-\sin \theta|020>+\cos \theta| 100\right\rangle,
\end{aligned}
$$

with $\theta \sim 45^{\circ}$ for ${ }^{12} \mathrm{CO}_{2}$. Because of this equal mixture of normal modes the transition rate for the two eigenstates $\psi_{\mathrm{u}}, \psi_{1}$ are nearly equal, and the two transitions have nearly equal strength in the absence of the discharge. With the discharge, we see that both bands decrease because of vibrational heating and depletion of the ground state, but the $\psi_{1}$ band at $1285 \mathrm{~cm}^{-1}$ is substantially decreased relative to $1388 \mathrm{~cm}^{-1}$. We observe a 1388/1285 ratio of from 8-10 for a broad range of pressures and discharge currents. This is a very surprising effect.

Since both bands begin at the ground state, one might be tempted to suggest that the transition rate is altered or the degeneracy of the harmonic states broken in the discharge; but we observe no shifts in the wavelengths of transitions as the discharge is turned on. The signal in CARS depends upon the difference in populations $\mathrm{n}_{\mathrm{f}}-\mathrm{n}_{\mathrm{i}}$ for the transition. If the discharge saturates the population of the final state at $1285 \mathrm{~cm}^{-1}$, then its intensity would be decreased relative to $1388 \mathrm{~cm}^{-}$ 1. This suggests an electron excitation rate for $\psi_{\mathrm{l}}$ which is greater than $\psi_{\mathrm{u}}$.

Zhu et al. observed exactly the opposite in their experiments. 66 They produced hot electrons by multiphoton ionization of $\mathrm{I}_{2}$ at $193 \mathrm{~nm}$. The resulting hot electrons preferentially populated $\psi_{\mathrm{u}}$ and by nearly a factor of 10 ! They measured the populations at $25 \mathrm{mT}$ using direct infrared absorption from the states $\psi_{\mathrm{u}}, \psi_{1}$ at $4.3 \mu \mathrm{m}$ which excites a mode of antisymmetric stretch, $101 \leftarrow 100$ or $021 \leftarrow 020$. Their results are nicely in agreement with Eq. 2.4 if one assumes that the electron perturbation $\langle 100|\mathrm{~V}| 000\rangle$ and $\langle 020|\mathrm{~V}| 000\rangle$ are nearly equal. Then the negative sign in the wavefunction of $\psi_{1}$ would cause a "quantum interference" in the electron excitation rate. Though $<020|\mathrm{~V}| 000>$ involves a two quanta transition, electron impact at low energies would be expected to be dominated by charge cloud polarization, and it is known that $\mathrm{CO}_{2}{ }^{-}$exists in a bent configuration. ${ }^{67}$ Hence excitation of bend though a $\mathrm{CO}_{2}^{-}$channel is expected.

Electron energies in a discharge are broadly distributed compared to the experiment of Zhu et al. and large populations of 010 are expected to be produced. We have observed the $110 \leftarrow 010$ hotband in the discharge. Electron excitation to $\psi_{\mathrm{u}}$ from 010 would likely be stronger for the component $\langle 020|\mathrm{~V}| 010\rangle$, hence both state should be populated with equal rates in a discharge with a large population of low energy electrons $(\langle\mathrm{E}\rangle \sim 0.1 \mathrm{eV})$.

${ }^{65} \mathrm{We}$ denote the number of quanta in each normal mode, listing (symetric stretch, bend, asymetric stretch).

66 Lei Zhu, Scott A. Hewitt, and George W. Flynn, J. Chem. Phys. 94, 4088 (1991).

67 A. R. Rossi and K. D. Jordan, J. Chem. Phys. 70, 4422(1979). 
This then leaves only relaxation rates for explaining the large population of $\psi_{1}$ relative to $\psi_{\mathrm{u}}$ in the discharge. Now the quantum interference for $\psi_{1}$ plays an important role. If collisions cause equal transition rates for the harmonic components $\mid 020>$ and $\mid 100>$ then $\psi_{1}$ will have a small relaxation rate to either lower level $\mid 000>$ or $\mid 010>$ compared to $\psi_{\mathrm{u}}$. The experiments of Zhu et al. confirmed the interference for collisions with both electrons and hot atoms, though suppression was greater for electrons. While the discharge is on relaxation occurs by both electron (superelastic collisions) and atomic collisions. When the discharge is off only atomic collisions play a role.

We examined the collisional relaxation rates by

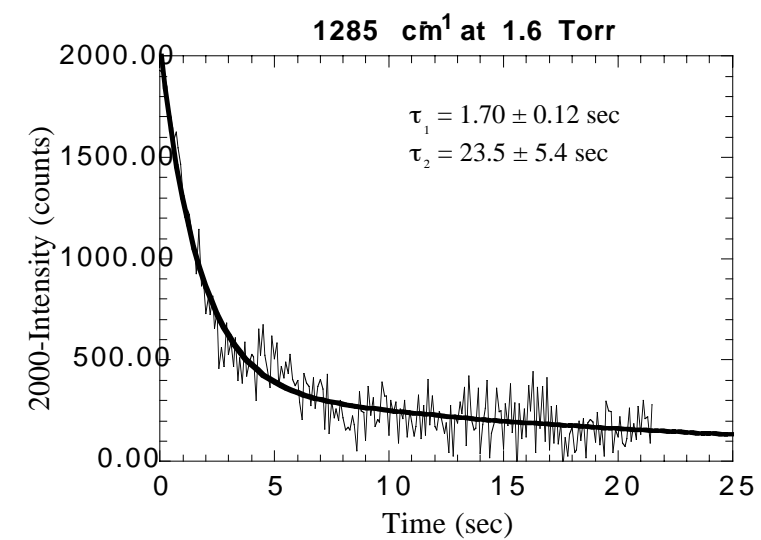

Fig. 2.10. Growth in intensity of the 1285 $\mathrm{cm}^{-1}$ band of $\mathrm{CO}_{2}$ plotted as a difference from the maximum count. The fit is shown as a solid line.

measuring the change in $1285 \mathrm{~cm}^{-1}$ and $1388 \mathrm{~cm}^{-1}$ signals as a function of time after the discharge is turned off. The rates were measured at pressures from $0.8 \mathrm{~T}$ to $4 \mathrm{~T}$. The $1388 \mathrm{~cm}^{-1}$ signal increased with an exponential time at $1.6 \mathrm{~T}$ pressure of $\tau \sim 2.0 \pm 0.3 \mathrm{~s}$. Since the population of $\psi_{\mathrm{u}}$ is small when the discharge is on, this growth results from atomic collisions repopulating the ground state. This vibrational relaxation time for replenishing the ground state decreases with increasing pressure as expected. The growth in $\psi_{1}$ is shown in Fig. 2.10, plotted as $\mathrm{I}_{0}-\mathrm{I}(\mathrm{t})$. We observe a two-exponent growth, with the shorter time constant consisted with the observed repopulation of the ground state. The other time constant must represent the vibrational relaxation from $\psi_{1}$. The measured time constant is an order of magnitude larger than for repopulation of the ground state. This result is consistent with a model of quantum interference for the vibrational relaxation rate from $\psi_{1}$ compared with the rate expected from $\psi_{\mathrm{u}}$.

The last possible explanation of the data would involve Raman saturation of the upper levels. We eliminated this possibility by measuring the 1388/1285 ratio for an order of magnitude change in the pump laser intensity. We observed no significant change in the intensity ratio.

\subsubsection{Experiments in the high pressures discharge}

Efforts were made to obtain the spectra of $\mathrm{N}_{2}+$ in the high pressure discharge. To prevent the production of larger clusters such as $\mathrm{N}_{3}{ }^{+}$in the discharge which would have decreased the signal from $\mathrm{N}_{2}{ }^{+}$, we added small pressures (10-100T) of $\mathrm{N}_{2}$ to the $4 \mathrm{~atm}$ He discharge. From modeling we expected helium ion and metastable densities greater than $10^{17} \mathrm{~cm}^{-3}$ in the discharge. If these molecules are lost by Penning ionization from the metastable and charge transfer from the ions, we predicted equally large concentrations of $\mathrm{N}_{2}{ }^{+}$. Assuming a Raman cross section similar to $\mathrm{N}_{2}$ this concentration should have yielded a sufficient CARS signal for detection with our apparatus.

Unfortunately we obtained laser induced breakdown with every pump laser shot. This breakdown was initiated by dust produced by sputtering of the discharge electrodes. We could eliminate this effect by lowering the pressure and repetition rate of the laser so that the dust would settle out of the focal volume of the Raman lasers in the time between shots. This required total pressures below $50 \mathrm{~T}$ for which the Raman signals were too small for detection. Removing the dust will require circulating the discharge gas through a small particle filter. Such filters are known in the aerosol community as diffusion batteries. They have high a high transmission $>90 \%$ for the gas, but collect nearly $100 \%$ of the particles. This experiment is on the threshold of success. 


\subsection{Single-particle spectra: Microluminescence and NSOM}

We have achieved single particle spectra of quantum dot islands using microluminescence, and efforts toward using NSOM are continuing. These experiments were done in collaboration of Prof. Ken Shih in our solid state group and were performed in my laboratory. Ken provided the expertise in scanning probe technologies and I provided the experience in lasers and optical spectroscopy. Quantum dot islands are grown by MBE within quantum wells. The process is similar to the nucleation of raindrops on a wetted, waxed car. The deposition is halted when the islands first form, and then are capped by the deposition of a semiconductor with a larger band gap. This process terminates the dangling bonds at the island's surface, eliminating the defect problems described above. There are disadvantages of these quantum dots. The islands have a trapezoidal structure with a great deal of stain at the interface, particularly at the corners. This makes calculation of the spectral fine structure very complex. The thick capping layer makes approach to the particle impossible, hence one cannot size the particle during NSOM, so that size correlated fine structure is obtained. Lastly, it is difficult to control the size and separation distribution for the particles.

The microluminescence instrument uses the spectral separation of the light emitted by the quantum dots to measure the centroid of the diffracted image. Thus the position of the nanoparticle along the slit of the spectrograph can be determined to $\pm 40 \mathrm{~nm}$, and we can correlate lines in the spectra to a particular dot even though many dots are localized within a distance smaller than the resolution of the microscope. 68

We have found that it is easier to chase a particle as it moves because of differences in expansivities of the sample stage during temperature changes when using the microluminescence apparatus rather than the current NSOM apparatus. We used this tracking ability to study the thermalization of carriers in quantum dots, reported in paper 18 of the publication list. Cleaved samples were observed on edge to study self-organized stacked layers of particles in isolated quantum wells. Observation of the fluorescence from the edge of the sample allowed us to study the stark effect on excitons in the quantum wells. This work observed what we have speculatively assigned to fluorescence from an exciton bound to two adjacent quantum dots separated by only a few nanometers., thus rapid tunneling of the excitation between particles is expected. By applying an electric field along the axis of this quantum-dot, the spectra of this quantum-dot molecule could be altered. This research was reported in Publications 19 and 21.

Microluminescence provides no information about the size of the excited particle, so we have continued with our development of a new Near-field Scanning Optical Microsope (NSOM). We were able to measure spatially selective, photoluminescence spectra of an AlGaAs/GaAs heterostructure using the instrument. The instrument we originally developed used optical feedback, a simpler approach than controlling the distance from the surface with AFM ${ }^{69}$ and ideally suited for building a UHV NSOM operating at low temperatures. We have built and tested a UHV version of this instrument which operates at temperatures as low as $2.5^{\circ} \mathrm{K}$ where phonon broadening of the photoluminescence can be essentially eliminated. By using this Low T-NSOM, we have observed light emission from individual quantum dots of nanometer dimension.

${ }^{68}$ H.Htoon, Hongbin Yu, D. Kulik, J. W. Keto, O. Baklenov, A.L.Holmes Jr., B.G. Streetman, and C.K.Shih, "Quantum dots at the nanometer scale: Interdot carrier shuffling and multiparticle states," Phys. Rev. B. 60, 11026-11029(1999).

${ }^{69}$ G. Guttroff, John W. Keto, C. K. Shih, A. Anselm, and B. G. Streetman, "A new method of scanning near-field optical microscopy and its Application to AlGaAs/GaAs Heterostructures," Appl. Phys. Lett. 68, 3620(1996). 

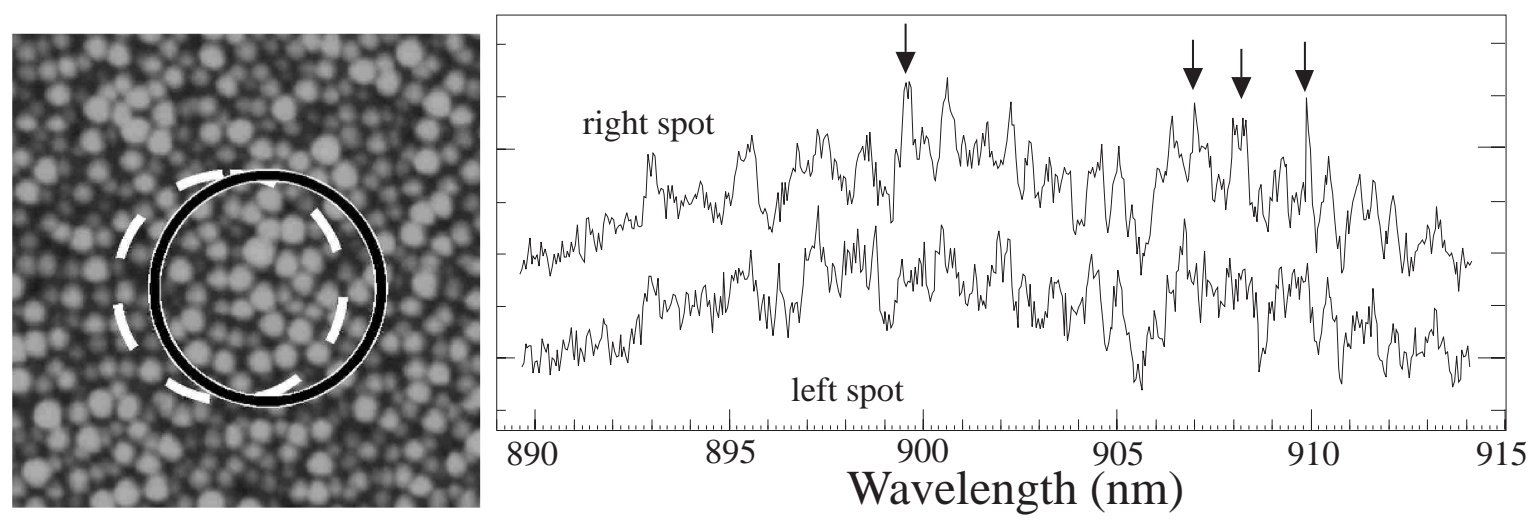

Fig. 8.1 Spatially resolved near-field scanning photoluminescence spectroscopy of individual quantum dots. The samples are InGaAlAs quantum dots formed under Stransky-Krastonov growth mode using molecular beam epitaxy.

Shown on the left in Fig. 8.1 is an AFM image of $30 \mathrm{~nm}$ InGaAlAs quantum dot samples before the GaAs capping layer was deposited. Note that the light from the NSOM tip ( $\sim 50 \mathrm{~nm}$ dia.) expands within the capping layer to a diameter on the order of $200 \mathrm{~nm}$. The NSOM measurement is performed on a sample with capping layers which are added to prevent oxidation of the quantum dots. Superimposed on the image are the two circles representing the two different NSOM probing areas which are $30 \mathrm{~nm}$ apart. The corresponding near-field photoluminescence spectra acquired from these two spots were shown on the right. One observes very sharp spectral features with a line width between $250 \mu \mathrm{eV}$ and $800 \mu \mathrm{eV}$, corresponding to the delta-function like density of states of individual quantum dots. The inhomogeneity of the quantum dots is directly reflected in the different spectral peaks. The majority of the spectral peaks appear in both spectra, reflecting the fact many of the quantum dots are in both probing areas. Some spectral peaks appear only on the upper spectrum (marked by arrows) representing the quantum dots which are only in the probing area on the right, while some peaks are absent, indicating that some quantum dots are no longer in the probing area.

Though the low-T NSOM was operated successfully, work was not productive. As in all probe technology images are obtained using a tube scanner. This device is coarsely positioned in our instrument using a piezoelectrically driven "walker" which moves using a slip-stick operation. The devices are capable of steps of a few $\mathrm{nm}$, but we found that when cycling the microscope from room to low temperatures, the step size varied significantly. Too many experiments resulted in a crashed tip, with a resulting long time required to warm the instrument, replace the tip, re-establish UHV, and then cool the instrument to $4 \mathrm{~K}$.

The second generation low-T NSOM kept the walkers at room temperature, and the sample was mounted to a cold finger surrounded by a heat shield. The optical fibers for both driving the fiber and scattered light detection are then inserted though a small hole in the heat shield. This approach has worked but will require modifications to cool the fibers, at least to the heat shield temperature. The newest version is a hybrid microscope that uses a pulled fiber tip for excitation and fibers for both distance control and spectra on the quantum dot side of the sample, but adds a microscope objective on the transmission side of the sample holder. The microscope images luminescent particles through an imagining spectrometer as in our microluminescence apparatus. The new NSOM also uses a UHV cold finger to achieve a base temperature of ( $4 \mathrm{~K})$ and a secondary heater for temperature studies. The design of the heater and sample holder compensates for thermal expansion so that the target particles will remain stationary to within $5 \mathrm{~nm}$ for $20 \mathrm{~K}$ changes in temperature. 
In addition to a complete redesign of the NSOM apparatus, we have completely redesigned the NSOM software. The original NSOM was programmed in DOS. We interfaced the A/D and $\mathrm{D} / \mathrm{A}$ operations using CAMAC ${ }^{70}$ controlled with a multiprocessor computer running the Linux operating system. This computer system will be shared in the future by all experiments in my laboratory, and replaces an antiquated PDP-11 system used previously. We are using the Kinetic System Model 2915 PCI CAMAC crate controller. We modified a PCI driver for controlling CAMAC by adding multiple crate and interrupt support to a driver previously written by a KEK high-energy physics collaboration. ${ }^{71}$ When the interrupt support is fully debugged, we will publish this driver for other CAMAC and Linux users. Using direct memory access, the system achieves $3 \mathrm{MByte} / \mathrm{s}$ data rates to block data devices. Using interrupt driven, software control of the NSOM we achieve image rates of $20 \mu \mathrm{s} / \mathrm{pixel}$, hence we can obtain 1000x1000 pixel images in approximately $20 \mathrm{sec}$. Graphics output and user interface to the software is provided by Labview. ${ }^{72}$ This allows students to easily modify and program instrument control. Two Physics undergraduates were responsible for the software development, Chrisopher Boyd developed the CAMAC driver and data acquisition library and Mike Beam developed the library connections to Labview and programmed the operation of the NSOM.

\subsection{Single particle spectroscopy of nanoparticles}

We extended our single particle spectroscopy to examine the spectra of isolated nanocrystals produced by Laser Ablation of Microparticles (LAM). Initially we found that semiconductor particles produced by LAM were highly chained. (See Fig. 8.2) We have found that for metal particles chaining is unlikely because of photoionization of the particles by the laser in their later stages of nucleation. The chaining in semiconductor systems results either from a more rapid recombination of the charge on the cluster, or that a higher charge state is required to prevent chaining of semiconductor clusters as compared to metals. If the particles are collected within a $5 \mathrm{~cm}$ of the laser ablation region, Fig. 8.2 TEM of CdSe chains.

little chaining occurs. We also found that particles can be collected at larger distances downstream for lower pressures of carrier gases. This is consistent with slower electron-ion recombination at the lower pressures.

We have now developed the capability of writing nanoparticles on substrates positioned on a substrate held in a UHV compatible chamber (though UHV is not maintained in during deposition, the substrated is only exposed to a high-purity, argon or helium, carrier gas. This new machine and its capabilities will be described in more detail in Sec 4. One version of the apparatus uses an electrostatic mobility analyzer to insitu narrow the size distribution of deposited particles. This analyzer separates the electrons and positively charged nanoparticles reducing the rate of recombination. We also find that this increases the distance downstream from the ablation region for the formation of chains.

\footnotetext{
${ }^{70}$ CAMAC is a modular IEEE standard interface for "Computer automated acquisition," and has been used extensively by nuclear and high energy physicists.

71 M.Haseno, Y.Tanaka, and Y.Nagasaka, Nagasaki Institute of Applied Science (NIAS), Nagasaki, Japan, 3Mar-1998, Modified by C.Timmer, US. Jefferson Lab.

${ }^{72}$ Labview is a graphical instrument interface written and sold by National Instuments Corporation.
} 
We show in Fig. 8.3 a sample image in dark field of single, silicon nanoparticles, deposited dry on sapphire. The circled particle scattered red light before a 10 second exposure to the green excitation laser. No fluorescence was seen from the particle during exposure. Initially we suspected quenching by defects but we know know from experiments described in Sec. 8.3 that the excitation wavelength was too long $(488 \mathrm{~nm})$ for effective excitation of excitons seen in Si particles produced by LAM.

In very recent calculations, Liu, et al. have calculated the effects of dangling bonds and hydrogen surface states on the energy levels of silicon nanoparticles. ${ }^{73}$ Surface defects partly fill the band near the edge of the HOMO, but a clean gap is present. However, if the surface state is allowed to reconstruct, they find the band completely fills because of the resulting strain

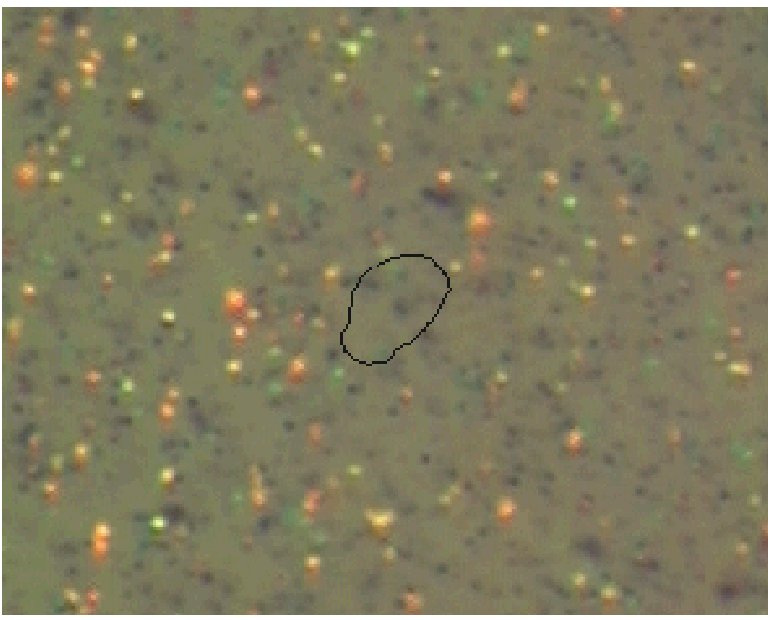

Fig. .8.3 Dark field image of scattered light of $\mathrm{Si}$ nanocrystals dispersed on a sapphire substrate. The circled particle has been "bleached" by a $10 \mathrm{~s}$ exposure of the laser at $10^{5} \mathrm{~W} / \mathrm{cm}^{2}$. which distorts the crystal structure throughout the cluster; the particle is essentially a metal. Hydrogen passivation after relaxation preserves this condition, but if the surface is passivated before the nanocrystal relaxes, the bandgap is nearly that of bulk silicon even after relaxation. ${ }^{74}$ We believed that synthesis by LAM might be a test for this model as the nanocrystals are ejected into the carrier gas at high temperature $(\sim 5000 \mathrm{~K})$ before cooling. If we add hydrogen, passivation may occur before crystal relaxation. (There is also a danger of rapid diffusion of hydrogen throughout the particle.)

We prepared samples of silicon nanoparticles produced by LAM in $\mathrm{He} / \mathrm{H}_{2}$ and pure $\mathrm{He}$ carrier gases. A second set of samples produced in pure He carrier gases were exposed to air after collection so they formed native oxide coatings; a third set of samples were first exposed to $\mathrm{H}_{2}$ at the collection wafers. All three types of particles were collected dry onto either Si wafers with stable native oxides, or onto flat and polished sapphire wafers. We have observed no fluorescence for all eight samples when observed with microluminescence! Again we now know that we needed a shorter wavelength excitation laser, though such a uv laser is incompatible with our microluminescence apparatus optics.

Since surface defects have proved to be less of a problem for CdSe nanoparticles, we made similar experiments from isolated individual particles collected on sapphire substrates. Charging and bleaching was not a problem and we found that $50 \%$ of the nanoparticles observed in dark field images similar to Fig. 8.3 were fluorescent. We did not observe the blinking of the particles observed by Bawendi, and coworkers, ${ }^{75}$ perhaps because of dry collection. An example spectrum is shown in Fig. 8.4 where we observed a broadened spectrum as expected for excitons at room temperature. Subsequent experiments attempting measure spectra of nanoparticles at low temperatures were not successful. Analysis of these particles by XRD using high-resolution TEM

\footnotetext{
${ }^{73}$ Lei Liu, C. S. Jayanthi, and Shi-Yu Wu, "Factor Responsible for the Stability and the Existence of a Clean Energy Gap of a Silicon Nanocluster," J. Appl. Phys. 90, 4143(2001).

${ }^{74}$ Lei Liu, C. S. Jayanthi, and Shi-Yu Wu, "Factor Responsible for the Stability and the Existence of a Clean Energy Gap of a Silicon Nanocluster," submitted to Phys. Rev. B, private comm.

${ }^{75}$ Shimizu, K.; Empedocles, S. A.; Neuhauser, R.; Bawendi, M. G. Stark spectroscopy investigation of spectral diffusion in single CdSe quantum dots Proc. - Electrochem. Soc., 98-19 (Quantum Confinement: Nanostructures), 1999, pp. 280-285
} 
demonstrated that the particles had become Cd rich over several weeks of storage (in argon) while waiting access to the microluminescence apparatus. Oxidation, even in an argon filled glove box, resulted in loss of $\mathrm{Se}$ in the form of $\mathrm{SeO}_{2}$.

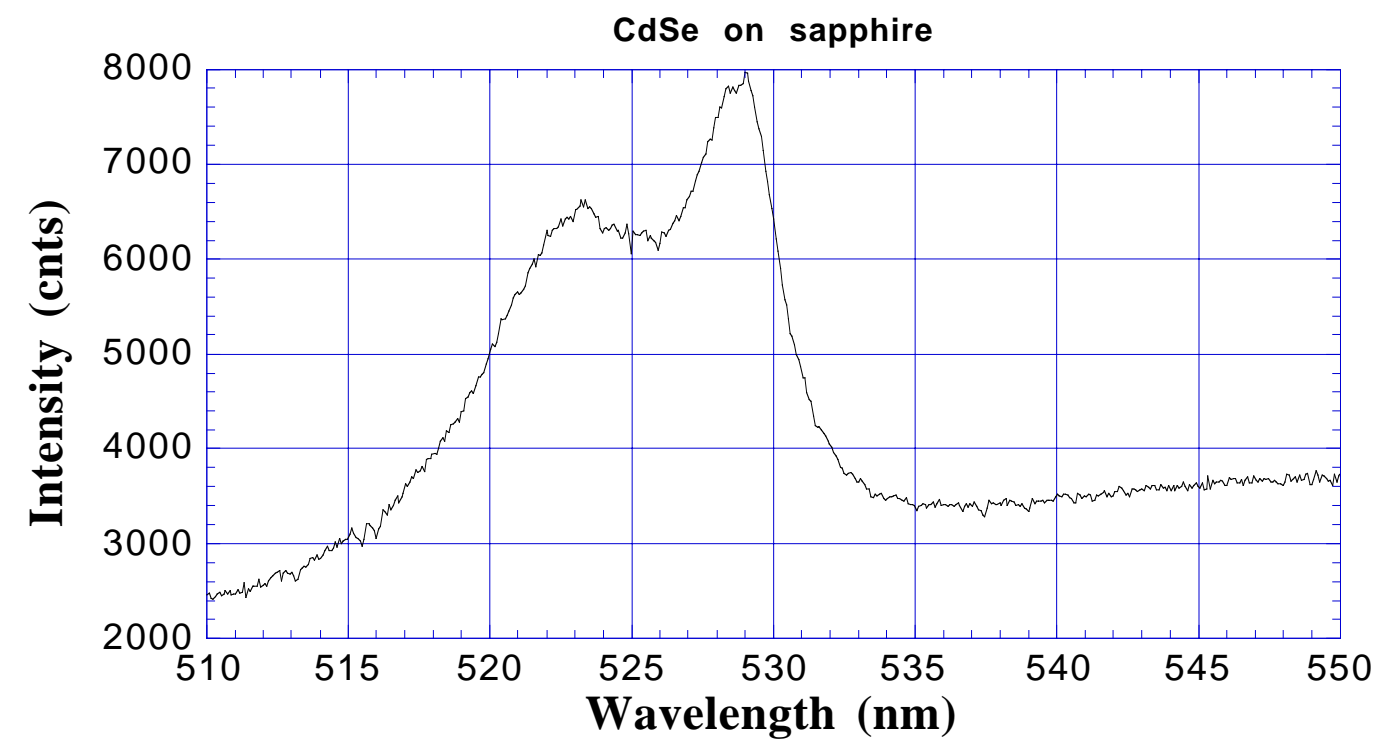

Fig. 8.4. Microluminescence of CdSe nanocrystals collected dry on sapphire substrates. The particles were produced in 1 atm of $\mathrm{N}_{2}$ and collected dry on sapphire substrates. The shoulder appearing at longer wavelengths results from sapphire fluorescence.

\subsection{Nanoparticles collected in a surfactant}

Although we were successful in obtaining fluorescence of CdSe nanoparticles when experiments are conducted soon after manufacture, we have been unable to obtain fluorescence for Silicon particles perhaps because of reactions with atmospheric gases. Bawendi and coworkers found that $\mathrm{CdSe}$ particles were stable and fluorescence $(\mathrm{QE} \sim 5 \%)$ for long periods when capped with tri-octylphosphine oxide (TOPO); hence we have pursued stabilization of both $\mathrm{CdSe}$ and $\mathrm{Si}$ nanoparticles in various surfactants. Success would broaden the applicability of our particles, enable size selective precipitation, and the formation of $2 \mathrm{~d}$ and $3 \mathrm{~d}$ lattices of nanoparticles by the use of attractive ligands.

Large quantities of nanoparticles can be produced by LAM and collected in flowing films of surfactant. These are then tested using a fluorimeter to determine the quantum efficiency for fluorescence and to measure the room temperature absorption and fluorescence spectra. For producing dense nanocrystal colloids, we first tried supersonic impaction into either nonanoic acid or a solution of $10 \%$ by mass Tri-octylphosphine oxide (TOPO) in anhydrous-butanol. In the case of nonanoic acid, which has a low vapor pressure, we can recirculate the solution through the collection region many times and hence produce very high number densities of nanocrystals in the liquid. We have demonstrated that the particles are spherical in shape and remain non-agglomerated even at densities of $1 \times 10^{18} \mathrm{~cm}^{-3}$ which corresponds to approximately $25 \%$ concentration of nanocrystals by mass. Nonanoic acid as a collection liquid has several interesting properties. First, the nonanoic acid molecule appears to have a strong affinity for the surface of the CdSe nanocrystal, however, the surface passivation is not complete, resulting in loose flocculation of the particles. This property is practically important because it allows nanocrystal solutions to be further concentrated from the collected solution and even "slurries" of nanoparticles can be produced 
approaching $50 \%$ mass density. Unfortunately, the incomplete surface capping leads to an inability to carry out size selective precipitation on the crystals.

We have experienced similar behavior with silver nanoparticles captured in nonanoic acid. ${ }^{76}$ (Publication 21) As produced, the sample solutions are opaque and a dark grey to black in color. If allowed to settle, a dense precipitate forms at the bottom of the test tube. We find, however, that we can stabilize the nanoparticles in solution by annealing the sample at temperatures as low as $75 \mathrm{C}$ for several hours. After dispersing the particles ultrasonically, a sequence of beautiful color changes occur with temperature, progressing from a muddy brown color, to dark orange, and translucent yellow. Finally the samples become as transparent as water, though the density of nanoparticles is $\sim 0.1 \mathrm{~g} / 25 \mathrm{ml}$. Once transparent it is difficult to remove the particles by even centrifugation. The measured light attenuation decreases by several orders of magnitude as a function of time and during the color change. By measuring the change in attenuation as a function of time at various temperatures, we find the reaction is first order with an activation energy of 41.8 $\mathrm{kJ} / \mathrm{mole}$. Because the Arrhenius plots do not correctly extrapolate to room temperature, we know that an irreversible activation occurs between room temperature and $75 \mathrm{~K}$.

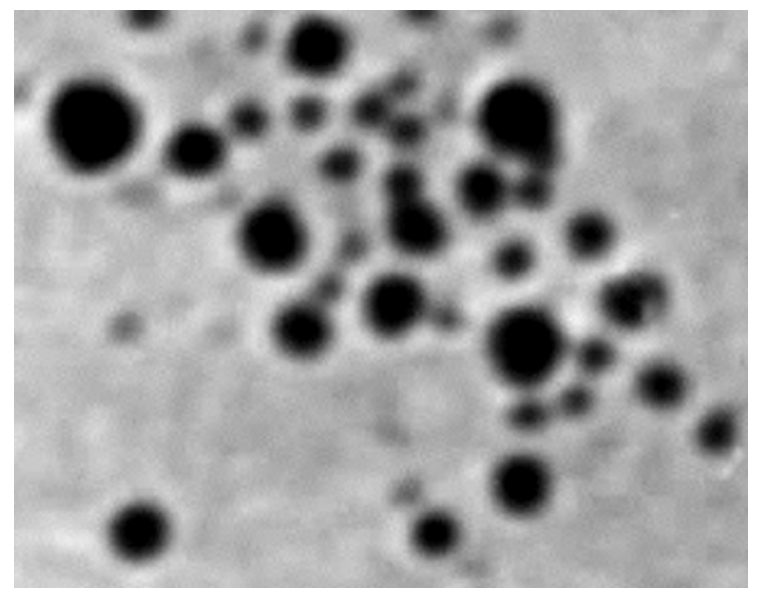

Fig. 8.5. TEM of silver nanoparticles collected in Nnonanoic acid.

Each nanoparticle (mean dia. $5 \mathrm{~nm}$ ) scatters by Rayleigh, scattering. The flocked particle (shown in Fig. 8.5) consists of hundreds of nanoparticles whose scattering is coherent since the overall dimension is still smaller than the wavelength of light. The scattering of the flocked particle then varies as the square of the number its nanoparticles N. Annealing the solutions initiates the breakup of the flocculants, and when the nanoparticles are stabilized as individual nanoparticles the scattering decreases by $\mathrm{N}$. The picture is supported by experiments measuring the dynamic light scattering DLS which measures by the dynamic drag of the particles their mean size. Shown in Fig. 8.6 is the mean size of the particles as a function of the time of annealing. The as collected flocked particles had a dimension of $600 \mathrm{~nm}$. This size decreases uniformly with time until of dimension unresolvable by DLS (dia $<40 \mathrm{~nm})$.

\footnotetext{
${ }^{76}$ Dale E. Henneke, William T. Nichols, Gokul Malyavanatham, Michael F. Becker and John W. Keto, "Surfactant stabilization of silver nanocrystals produced by laser ablation of microspheres," submitted J. Chem. Phys.
} 


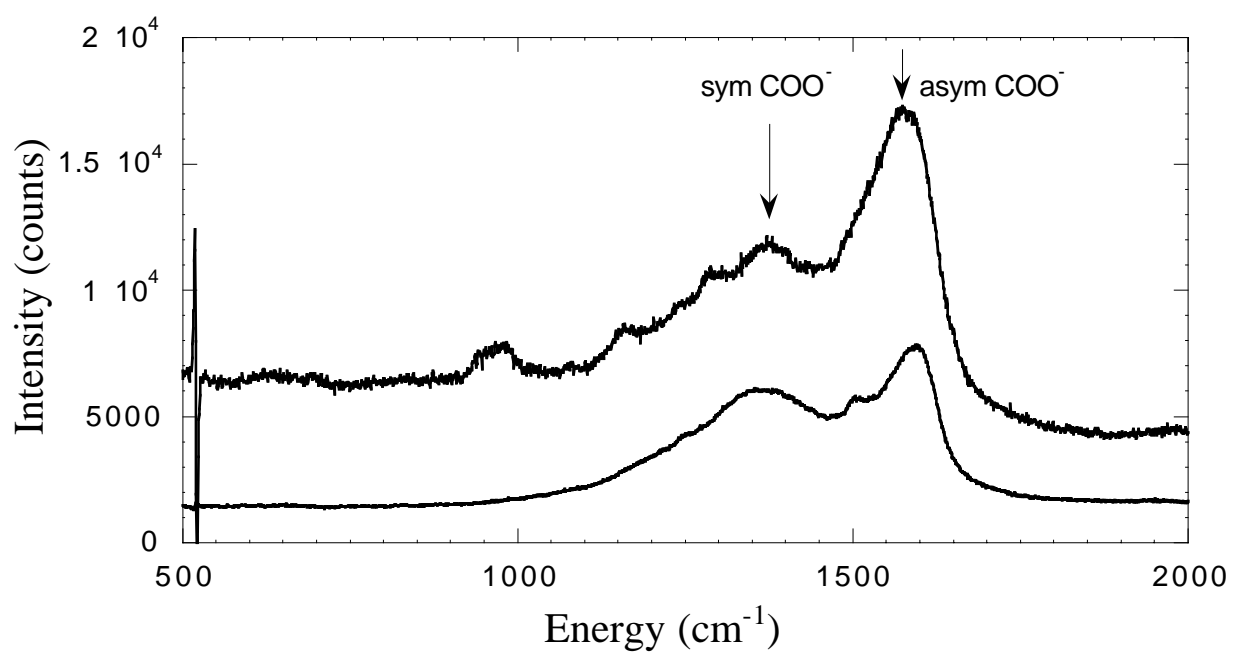

Fig. 8.7. SERS for nonanoic molecules on silver nanoparticles. The top curve is for an as collected sample and the bottom curve is for a sample annealed at $115 \mathrm{C}$ for $3 \mathrm{hr}$. The sharp spike near $500 \mathrm{~cm}^{-1}$ is a remnant from subtracting the Raman spectrum from the silicon sample wafer.

We believe the successful stabilization of the particles results from a change in the orientation of the surfactant molecule on the surface of the particle. We believe that the molecule initially attaches with the carbon backbone lying along the surface, thus the molecule covers nearly 10 surface sites. This inclined coverage allows the nanoparticles a closer distance of approach than if the surfactant molecules covered the surface with their backbone nearly parallel to the surface normal. Because of the closer distance and greater attraction between the nanoparticles, they bind to form the observed flocculates.

The $\mathrm{COOH}$ group of nonanoic acid may initially form a bond between the oxygen atom and a single silver atom in the surface similar to a carboxoylic salt $\mathrm{Ag}-\mathrm{OCOH}$. The dissociation energy for the hydrogen atom then produces a barrier for $\mathrm{COO}^{-}$to bind symmetrically to two silver sites as observed on silver surfaces. ${ }^{77}$ The initial asymmetric binding of the $\mathrm{O}=\mathrm{C}-\mathrm{OH}$ group would cause a significantly larger angle of the molecule relative to the surface normal allowing attraction of the hydrocarbon backbone to the surface of the nanoparticle. The activation energy of 0.44 $\mathrm{eV} /$ molecule then results both from breaking the attraction to the surface as well as the barrier to displacing the $\mathrm{H}$ atom Additional molecules can then react with the uncovered surface sites.

${ }^{77}$ E. L. Smith and M. D. Porter, J. Phys. Chem. 97, 8032(1993). 
This model is supported by surface enhanced Raman scattering (SERS) measurements of the nonanoic molecule on silver nanoparticles shown in Fig. 2.7 for unannealed and transparent samples. Two strong features are apparent in the figure, the asymmetric and symmetric stretch modes of the $\mathrm{COOH} / \mathrm{COO}^{-}$group of the molecule which is bound to the surface. Because of its chemisorption to the surface it has a larger enhancement than the other hydrocarbon modes in the figure. On silver nanoparticles there is an additional enhancement due to a resonance between the electronic virtual state of the molecule excited by the scattered photon and the plasmon mode of the nanoparticle 78 Rapid exchange of the excited electron with the metal also appears to broaden the transition. Note that the asymmetric mode is not observed for silver surfaces but has been observed on both copper and aluminum surfaces. ${ }^{79}$ On flat silver surfaces the absence of this mode has

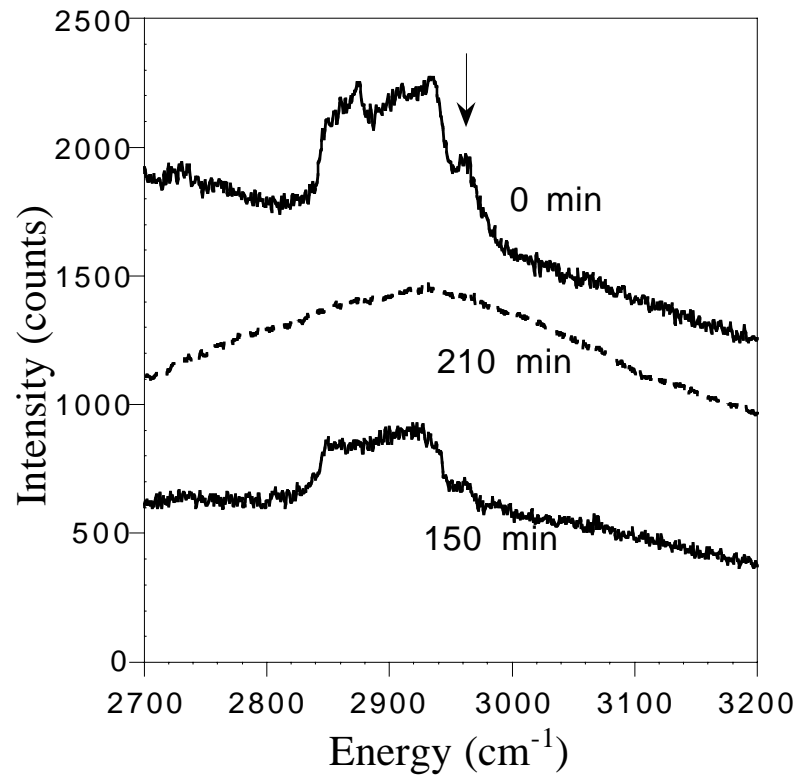

Fig. 8.8 CH stretching region of nonanoic acid measured for different annealing times. The arrow indicates the asymmetric stretching frequency of the methyl group. been used to argue that the $\mathrm{COO}^{-}$group of nonanoic acid is bound by the oxygen atoms equally to two different surface sites, forming a symmetric isosceles structure. ${ }^{80}$ The asymmetric mode observed in our spectrum indicates that the $\mathrm{COO}^{-}$bond has a larger angle relative to the surface normal compared to flat surfaces. This is consistent with the picture of the backbone lying on the surface of the nanoparticle. As the sample is annealed, this mode becomes weaker compared to the symmetric mode indicating that the angle relative to the surface normal becomes smaller. Also observed in the annealed sample are distinct peaks in the region $1200-1300 \mathrm{~cm}^{-1}$ assigned in the surface experiments to the $\mathrm{CH}_{2}$ wagging modes of the backbone of the molecule. The peak at 950 $\mathrm{cm}^{-1}$ was observed in the SERS spectra of carboxylic acids in silver sols and was assigned to the C$\mathrm{COO}^{-}$stretch. ${ }^{81}$ The hydrocarbon modes decrease in intensity with increasing annealing times and are completely absent in the fully annealed samples.

Also observed in the SERS spectra are the $\mathrm{CH}$ stretching modes of the backbone, shown in Fig. 8.8 for three annealing times. The spectrum of the as collected sample is nearly identical to that observed by Moskovits, et. al. The arrow indicates the clearly resolved C-H stretch mode of the $\mathrm{CH}_{3}$ end group of the hydrocarbon chain. Note that these peaks completely disappear as the solution is annealed. As more of the molecules stand vertically from the surface these modes are further from the surface and are less enhanced; hence they disappear.

We have collected silver nanoparticles in nonanoic acid dissolved in dowlanol (a proprietary solvent). We get very different qualitative behavior, indicating the surface state of the surfactant molecule is dependent upon the solvent.

\footnotetext{
78 Amy M. Michaels, M. Nirmal, and L. E. Brus, J. Am. Chem. Soc.121, 9932(1999).

${ }^{79}$ Yu-Tai Tao, J. Am. Chem. Soc. 115, 4350.

${ }^{80}$ E. L. Smith and M. D. Porter, J. Phys. Chem. 97, 8032(1993).

${ }^{81}$ M Moskovits and J. S. Suh, J. Am. Chem. Soc. 107, 6826(1985).
} 


\subsection{Semiconductor nanoparticles in surfactants}

Spectra of both unannealed and annealed suspensions of CdSe particles in nonanoic acid were measured, and we observed only weak fluorescence. Perhaps the strong surface interaction of $\mathrm{CdSe}$ with nonanoic acid results in a non-fluorescent surface trap state. A system which has been demonstrated to provide excellent passivation of the CdSe nanocrystal surface is Trioctylphosphine oxide (TOPO) in anhydrous-butanol. ${ }^{82}$ The TOPO binds to the surface and can provide enough steric hinderance to attractive van der Waals forces that the particles remain unflocculated. Difficulties are encountered with butanol in our vacuum system because butanol's room temperature vapor pressure is 10 Torr resulting in extensive evaporation during collection. The TOPO crystallizes on the deposition surface leading impaction of the nanocrystals into solid TOPO. The particles can be collected at very high densities in this way, however, attempts at redispersal of the solid TOPO-nanocrystal material in butanol have not been successful, and the nanocrystals flocculate quickly and settle out of solution. Sonication of the flocculants did not improve the stability of the solution and therefore the particles appear to be agglomerated. We believe that impaction into solidified and non-flowing TOPO does not allow the particles to be fully passivated. Octanol, with its lower vapor pressure, is now being used as the TOPO solvent.

The second issue we are addressing is the possibility that oxidation of the CdSe nanocrystals destroys their optical properties. Alivisatos and coworkers have shown that TOPO capped particles suffer preferential oxidation of Se from the surface of the crystal. $\mathrm{SeO}_{2}$ was found to be volatile. It is believed that the TOPO molecule binds primarily to the $\mathrm{Cd}$ on the surface, leaving the Se exposed to oxygen which diffuses through the TOPO capping layer. To combat this problem we hypothesized that a hydrogen capping layer on the bare $\mathrm{CdSe}$ nanocrystals would lead to a surface more resistant to oxidation.

To test this hypothesis we injected $4 \%$ by volume of Hydrogen into the microparticle carrier gas so that it reacts with the nanocrystals as they are ejected from the exploding microsphere into the aerosol carrier gas. The particles readily react when

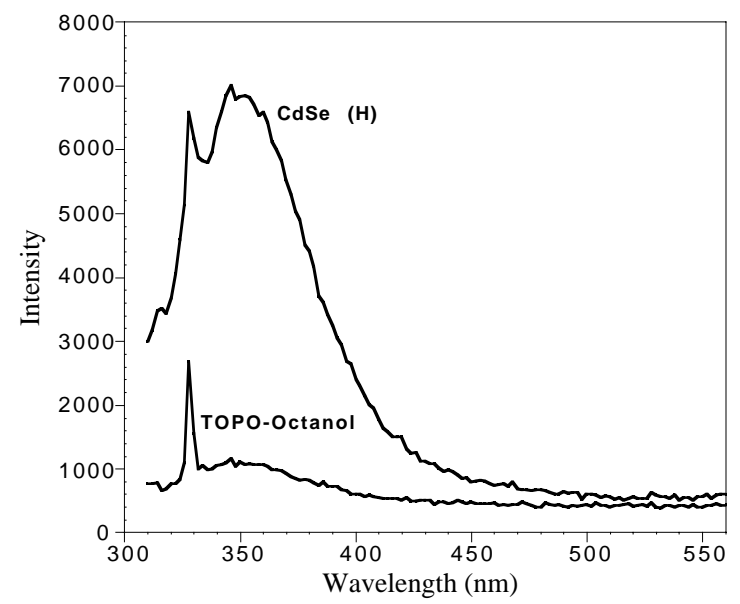

Fig. 8.9. Spectra of hydrogen capped CdSe nanoparticles collected in TOPO/octanol. The lower spectrum of the solvent without particles results from Raman scattering of the $300 \mathrm{~nm}$ excitation lamp. injected hot $(\mathrm{T} \sim 1000 \mathrm{C})$ into the cooling laser plasma. These particles are then supersonically deposited directly onto a carbon coated transmission electron microscope (TEM) grid and imaged in a JEOL 2010 high resolution TEM equipped with an energy dispersive x-ray fluorescence spectrometer (EDS) to determine elemental composition from small areas of the sample. Preliminary results have been encouraging and indicate that the hydrogen enhances oxygen resistance of the dry particles significantly. The hydrogen capping begins to breakdown after about four days when stored in ambient air while the non-hydrogen capped samples loose significant amounts of Se by the second day.

Finally, we have obtained fluorescence from TOPO/octanol solvated CdSe nanocrystals produced by LAM and capped with hydrogen. A spectra of nanoparticles produced in a helium/hydrogen aerosol is shown in Fig. 8.9. The fluorescent wavelength shown in Fig. 8.9 is

${ }^{82}$ C. B. Murray, D.J. Norris \& M.G. Bawendi, "Synthesis and characterization of nearly monodisperse CdE( $\mathrm{E}=\mathrm{S}, \mathrm{Se}, \mathrm{Te})$ semiconductor nanocrystallites," J. Am. Chem. Soc. 115, 8706-8715(1993). 
significantly shorter than that observed in Fig. 8.4. This blue shift is consistent with a mean particle diameter near one $\mathrm{nm}$, which is also consistent with sizes observed using high resolution TEM of particles collected on cargon grids during the same run. ${ }^{83}$ Both spectra demonstrate a significant quantum confinement of the excited electron. We next collected $\mathrm{CdSe}$ in argon producing nanoparticles with a mean size near $8 \mathrm{~nm}$. These particles had a fluorescence spectrum similar to Fig. 8.9, suggesting that the fluorescence is not size selective. We speculated that the spectrum results from super-enhanced Raman scattering SERS of the TOPO molecules capping the nanoparaticle. To test this speculation, we collected Ag nanoparticles in TOPO/octanol samples and again measured a fluorescence spectrum similar to Fig. 8.9. We have not yet made SERS measurements using the micro Raman apparatus to verify this interpretation.

\section{4 .1 Silicon nanoparticles captured in ethylene glycol}

$\mathrm{Si}$ nanocrystals (NC) were produced and collected dry on carbon films and as suspensions in ethylene glycol. The NC were produced both in a pure helium aerosol and a $\mathrm{He} / 10 \% \mathrm{H}_{2}$ aerosol. In recent studies of $\mathrm{Si}$ nanocrystals formed by ion implantation in $\mathrm{SiO}_{2}$, Min, et al. found annealing samples after implanting hydrogen not only removed the fluorescence from defects in implanted $\mathrm{SiO}_{2}$, it also significantly increased the quantum efficiency for size selective fluorescence centered at $780 \mathrm{~nm} .{ }^{84}$ They surmised that the diffusion of hydrogen to the $\mathrm{Si}$ nanocrystal surface passivated dangling bonds. We hoped that capping the dangling bonds at the creation of the particle would similarly prevent nonradiative trap states at the surface. HRTEM of the samples collected on carbon films demonstrated that the particles were crystalline and of mean size $\sim 3 \mathrm{~nm}$. Solvents used in preparing

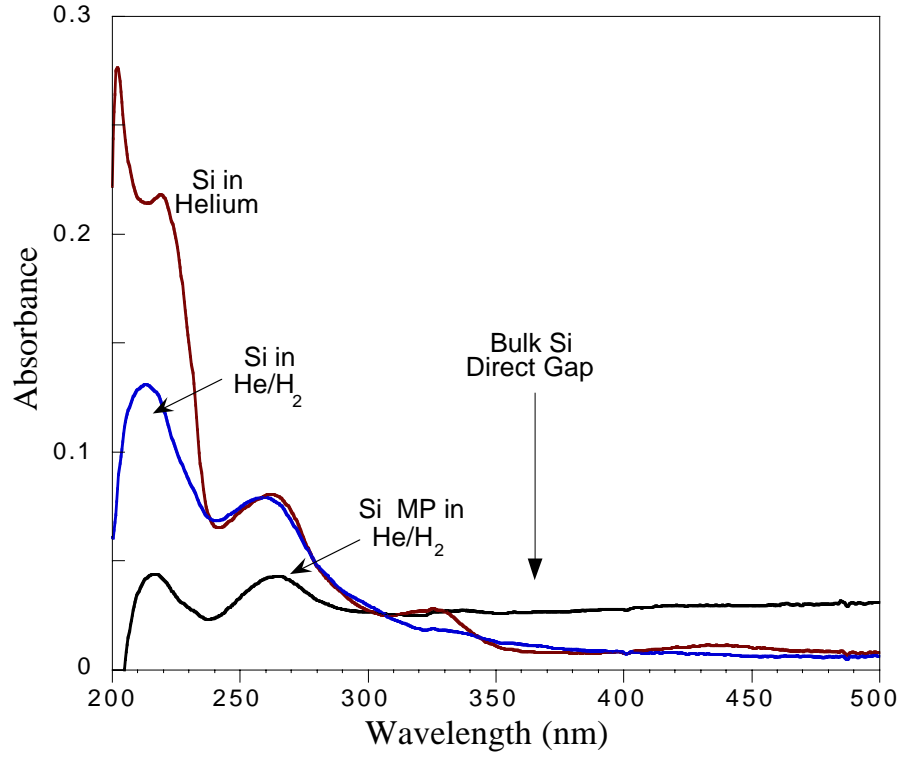

Fig. 8.10. Absorption spectra for Silicon nanoparticles in ethylene glycol prepared at a laser fluence of $1.8 \mathrm{~J} / \mathrm{cm}^{2}$. A spectra for a sample of presumably microparticles (Si MP) prepared without the laser is included. The direct bulk band gap for Silicon is indicated at $365 \mathrm{~nm}(3.4 \mathrm{eV})$.

the suspensions were degassed in vacuum to remove most of the dissolved gases. An "as made" UV-visible absorption spectra is shown in Fig. 8.10. The absorption spectra of the silicon microparticles (MP) are also shown in Fig. 8.10 with the known $\Gamma_{25}-\Gamma_{15}$ direct gap band edge. The spectrum of the MP are similar to that seen for bulk silicon. The absorption edges for

${ }^{83}$ William T. Nichols, Gokul Malyavanatham, Dale E. Henneke, James R. Brock, Michael F. Becker, John W. Keto, and Howard D. Glicksman, "Gas and Pressure Dependence for the Mean Size of Nanoparticles Produced by Laser Ablation of Microparticles in Aerosols", J. of Nanoparticle Res. 2, 141-145(2000).

84 K. S. Min, K. V. shcheglov, C. M. Yang, Harry A. Atwater, M. L. Brongersma, and A. Polman, "Defectrelated versus excitonic vilible light emission from ion beam synthesized Si nanocrystals in $\mathrm{SiO}_{2}$," Appl. Phys. Lett. 69, 2033(1996). 
nanoparticles formed in both aerosols are similar to that observe by Wilcoxon, et al. ${ }^{85}$ for $2 \mathrm{~nm}$ diameter NC grown in inverse micelles. Note the reduction in the direct gap absorption for the hydrogen prepared sample. Perhaps the silicon core diameter is much smaller for these particles giving a smaller absorption cross section. In general the absorption cross sections seen in Fig. 8.10 are very small. For the estimated concentration of Si NC we calculate an absorption cross section of $10^{-17} \mathrm{~cm}^{2}$ at $250 \mathrm{~nm}$, nearly $10^{-2}$ that of the Mie absorption calculated for similarly sized metal nanoparticles.

Photoluminescence spectra were taken with $300 \mathrm{~nm}$ wavelength excitation. Surprisingly, only the Si-nc sample prepared in pure helium exhibited weak PL. Spectra are shown in Fig 8.11 for Si-nc and hydrogen capped nc. The nanoparticle sample shows a PL peak around 340-350 nm. Figure 8.12 shows a plot of the emission spectra for the Si-nc sample with Raman and Rayleigh scattering from the ethylene glycol and nanoparticles removed. Note that excitation just above the absorption feature leads to an emission near this absorption edge. This result is very similar to that of Wilcoxon et al. They attribute the uv emission to the direct band gap recombination which is blue-shifted due to quantum confinement of the small particles. They also observed a small absorption feature around

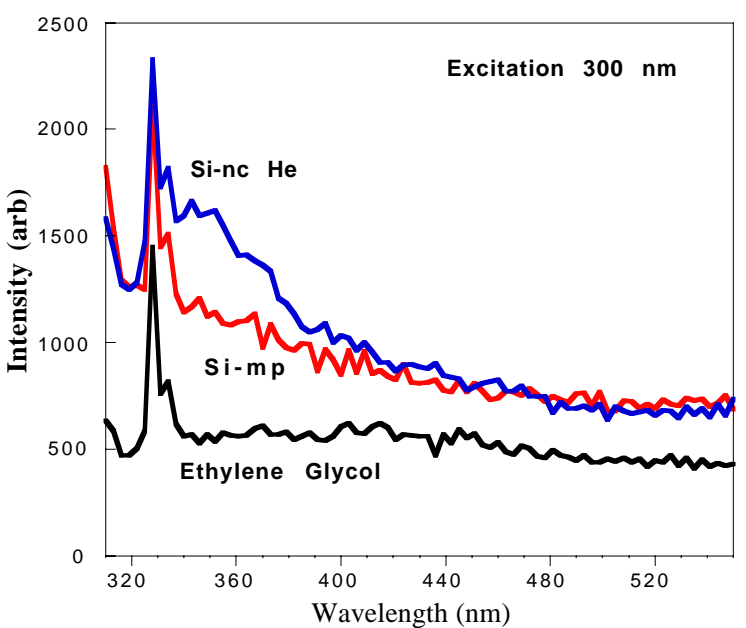

Fig. 8.11 Photoluminescence spectra of Si-nc with comparison to the no laser (Si-mp) sample and ethylene glycol. Note all particle samples exhibited very low PL.

$500-550 \mathrm{~nm}$ that yielded a smaller emission near $600 \mathrm{~nm}$ which was attributed to surface or defect recombination because its position was independent of mean size. Our fluorescence efficiency is about an order of magnitude less than Wilcoxon's, perhaps due to the longer excitation wavelenth of $300 \mathrm{~nm}$ vs their $245 \mathrm{~nm}$. As seen in the absorption spectrum significantly greater absorption is expected at shorter excitation wavelengths. (Unfortunately we had time on the fluorimeter before measuring the absorbance.) The weakness in fluorescence is both due to the QE and the small absorption cross section.

The very weak fluorescence at $665 \mathrm{~nm}$ is shorter in wavelength and narrower than that observed by Min, et al. ${ }^{86}$ We do not see emission at the $600 \mathrm{~nm}$ wavelength assigned to defects by both Wilcoxon, et al. and Min and coworkers. The shorter wavelength for our indirect gap fluorescence is consistent with having a smaller mean size (with narrower distribution) than estimated by Min, et al.

Initially we attributed the low PL quantum efficiency to surface defects. We carried out an anneal at $120 \mathrm{C}$ for 3 hours, and the features of the absorption spectra disappeared completely.

85J. P. Wilconxon, G. A. Samara, and P.N. Provencio, "Optical and electronics properties of Si nanoclusters sythesized in inverse micelles, Phys. Rev. B 60, 2704 (1999)

${ }^{86}$ Min, et al., ibid. 
Visual aspects of the sample all remained unchanged, but the PL spectra had a negligible intensity and the same qualitative shape as for pure ethylene glycol EG.

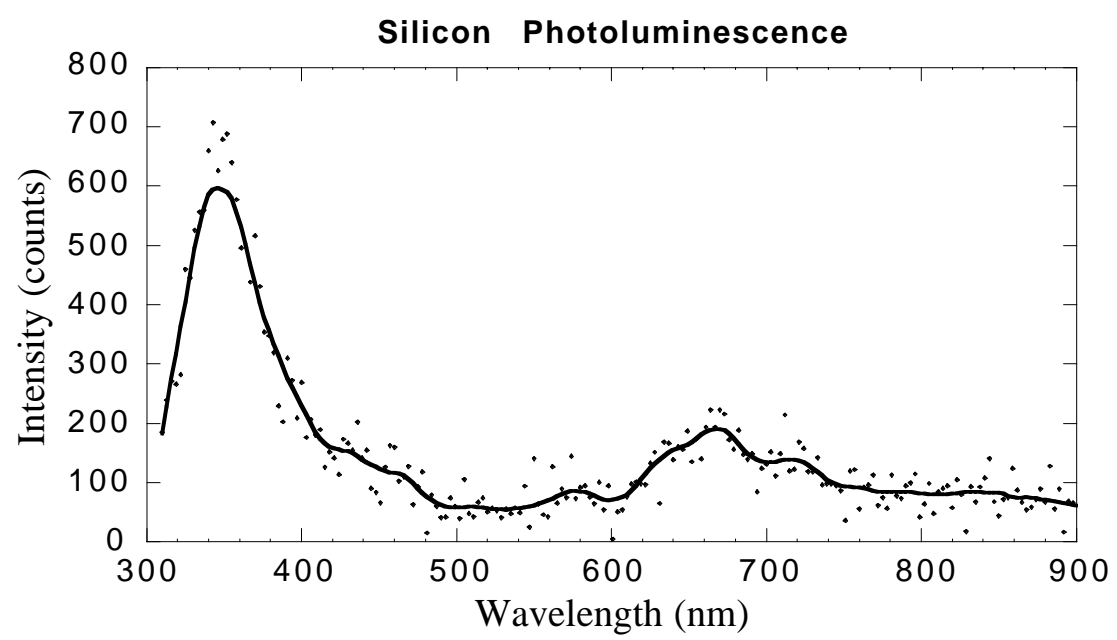

Fig 8.12. Plot of silicon nc luminescence. The PL spectra was obtained by subtraction of the ethylene glycol spectra (Raman peak) from the Si-nc data. A "smoothing" fit was applied as an aid to the eye .

Another possible contributor to the poor QE may be dissolved water vapor or oxygen in the EG. The increased temperatures of the anneal will oxidize the nanocrystals making the small ones $(2 \mathrm{~nm}) \mathrm{SiO}_{2}$ and not luminescent at all. This would explain the dramatic change in the absorption spectra. It is surprising, though, that the oxygen doesn't produce fluorescence of surface trap states in the $600 \mathrm{~nm}$ spectral range for the larger nanoparticles as has been observed by others.

Another possibility for the lack of fluorescence from the indirect gap was recently suggested by Zunger and coworkers. ${ }^{87}$ He found that dipole forbidden excitons are lower in energy than radiatively allowed states and that the splitting of these states increases to $30 \mathrm{meV}$ for the smaller, spherical quantum dots. Brongersma, et al., ${ }^{88}$ measured the fluorescence lifetimes for their $800 \mathrm{~nm}$ continuum as a function of temperature. They observed a shortening of the lifetime at increasing temperature and extracted a singlet-triplet energy gap consistent with that calculated by Zunger. The measured average radiative rate at room temperature was $3 \times 10^{5} \mathrm{~s}^{-1}$. signficantly smaller than expected for the direct gap transition.

We repeated these experiments measuring PL at excitation wavelengths varying from 250 to $500 \mathrm{~nm}$. We used anhydrous and oxygen free solvents and handled the solvents in glove boxes to eliminate the questions of impurtities from those sources. We measured a spectrum similar to Fig. 8.12 except that the main PL peak was shifted to $400 \mathrm{~nm}$. This shift to longer wavelengths is consisted with a larger Si core which likely resulted from less oxidation of the Si NP produced in the LAM process.

${ }^{87}$ F. A. Reboredok, A. Franceschetti, and A. Zunger, "Dark excitons due to direct interactions in silicon quantum dots, Phys. Rev. B 61, 13073(2001)

${ }^{88}$ M. L. Brongersman, P.G. Kik, A. Polman, K. S. Min, and Harry A. Atwater, "Size-dependent electronhole exchange interaction in Si nanocrystals," Appl. Phys. Lett. 76, 351(2000). 


\subsection{Impurity Doped Nanoparticles}

A recent goal was to develop techniques for the synthesis of nanocomposite and nanoheterogeneous materials and devices that combine the functional advantages obtained from the "size-tunable" properties of nanocomposite materials with the fabrication and direct-write advantages available from nanoparticles (NPs) manufactured by Laser Ablation of Microparticles (LAM) entrained in an aerosol. These materials are often found to have size tuneable optical properties and very large nonlinear susceptibilities. The fabrication of functional devices based on these materials requires the ability to assemble individual nanoparticles in micrometer patterns in a way compatible with current planar semiconductor technology. We have produced both Si NP in $\mathrm{SiO}_{2}, \mathrm{Si}$ in $\mathrm{AlO}_{3}$ and GaN NP in AlN. Unlike in Stransky-Krastonov (SK) quantum dots, however, the nanoparticle lattice is not necessarily indexed to the lattice of the substrate and the defect density at the particle interface may be high. These defects may quench exciton fluorescence at the interfaces.

One solution to the problem of interface defects, first achieved by Bawendi for CdSe nanoparticles is to shell the core particle during the growth phase. ${ }^{89}$ He successfully shelled $\mathrm{CdSe} \mathrm{NP}$ with $\mathrm{ZnS}$ and $\mathrm{ZnSe}$ materials. If the interface within the shell can be grown defect free and prevents the exciton from reaching the surface of the NP the exciton is protected from the environment outside of the NP. Another approach would be to trap the exciton within the NP on an impurity site. Hole donors may trap the hole within the bulk of the nanoparticle. Since the excitation energy for the impurity is below the bandgap, the exciton should trap at the impurity site and not on surface defect states, which have been shown not to be within the gap for some materials. If this site is sufficiently distant from the surface, the exciton would be protected from defects at the surface. Naturally this may prevent tuning of the exciton energy by confinement since the size of the electron wavefunction would have to smaller than the NP, but other benefits of the NP such as low temperature scintering and low scattering cross section are retained. The latter enables the composite, nanostructured material to have a high optical quality. If surface defect states are hole traps, hole donor impurities would trap the hole within the NP and an excited electron correlated to the trapped hole on the impurity would be confined and shifted in energy much like the band gap of the particle. Impurity states with large transition rates could then be used for high QE efficient PL devices, or states with long radiative lifetimes for stable light amplification could be selected (such as Ti or Er).

\subsubsection{Impurity doped nanoparticles}

Doped nanoparticles should in principle be possible using LAM by beginning with the appropriately doped micron sized powders. Possible impurities include $\mathrm{B}, \mathrm{Er}, \mathrm{Nd}, \mathrm{Ti}$, and $\mathrm{Cr}$ impurities in both $\mathrm{Si}$ and $\mathrm{GaN}$ nanoparticles. We have recently used ball milling to produce PZT micropowders with varying compositions as the starting material for LAM. Metal impurities might be added to semiconductor powders by diffusion or with the appropriate collaboration, ion implantation can be used to deposite metal ions in a semiconductor powder. For experiments reported here, we have used Er doped Phosphate glass powders as a source of microparticles for nanoparticle generation. The Er concentration in this powder was near saturation of the bulk material $(5.7 \pm 1.4$ at. \%). This concentration is sufficiently large that at least a few impurity atoms should be left within the nanoparticle.

${ }^{89}$ M. Danek, K. F. Jensen, C. B. Murray, and M. G. Bawendi, Chem. Mater. 8, 173(1996). 
The microparticles were captured in the aerosol gas using a fluidized-bed powder feeder. The starting powder consisted of irregularly shaped pieces with dimensions on the order of 1-10 $\mu \mathrm{m}$. Er doped fiber lasers normally emitt at $1535 \mathrm{~nm}$ and confinement would push the transition toward $1000 \mathrm{~nm}$. For applica-tions involving the communication industry it would be an advantage to produce NPs which are not shifted relative to the bulk, so the microparticles were entrained in an argon aerosol in order to produce a mean size of the particles on the order of $20 \mathrm{~nm} .90$ Following ablation nanoparticles were collected by supersonic impaction onto carbon TEM grids for size and structure analysis or onto quartz substrates for optical studies. Threshold laser fluence for laser ablation was found to be on the order of $1 \mathrm{~J} \mathrm{~cm}^{-2}$. Like for experiments with $\mathrm{PZT}^{91}$ the breakdown threshold was not sharp as

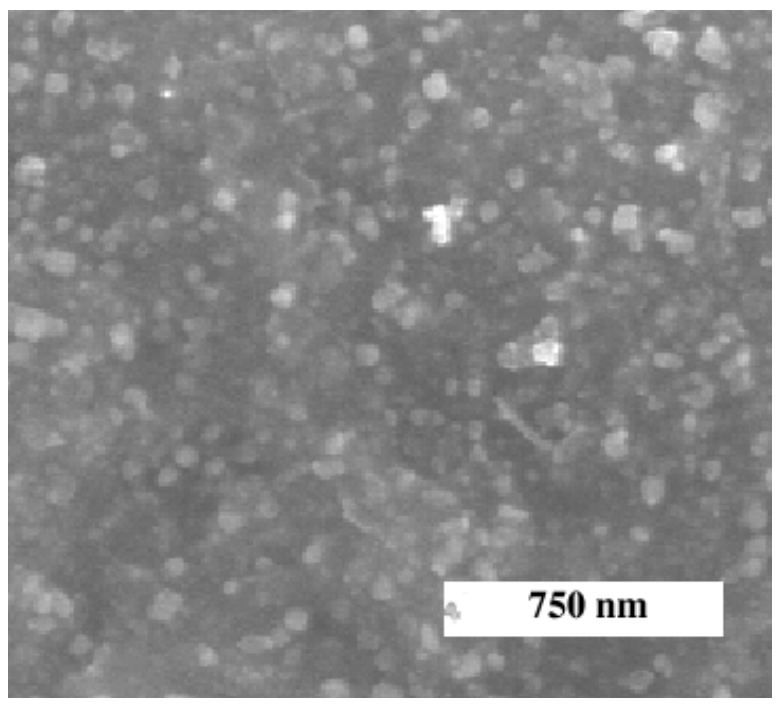

Fig. 8.13. SEM image of a dense deposition of Er doped phosphate glass NPs.

it is for metals and semiconductors. We attributed this both to the irregular shape of the particles and to their poor optical absorption which makes shock production within the particle difficult. The production efficiency of NPs using LAM with this material would be greatly improved by coating the microparticles with Er metal.

Shown in Fig. 8.13 is an SEM of a region where the nanoparticles were collected with sufficient density on the quartz substrate to form a nanostructured film. The particles lack sharpness because of charging of the glass and a lack of resolution for SEM The composition of the particles, averaged over an area of many particles, was measured using energy dispersive spectroscopy (EDS) in the SEM. We found a 50\% deficiency for $\operatorname{Er}(2.8 \pm 1.4$ at. \%), $\mathrm{Yt}(5 . \pm 2$ at. \%) and $\mathrm{La}(4.8 \pm 3.4$ at. $\%$ ) and a $20 \%$ increase for $\mathrm{P}$ atoms in the NP relative the the starting powder. We also found evidence in High resolution TEM micrographs of NP of segregation. Small NP particles $(\sim 4 \mathrm{~nm}$ dia) of relatively high contrast was found within the nanostructured films. A similar behavior was found for the production of dense deposits of PZT NP and were attributed to the weak shocks formed in the microparticles. Uniformity in the quality of PZT and the current glass materials may benefit from thin metal coatings of the starting microparticles.

\footnotetext{
${ }^{90}$ W. T. Nichols, G. Malyavanatham, D.E. Henneke, J.R. Brock, M.F. Becker, J.W. Keto \& H.D. Glicksman, "Gas and pressure dependence for the mean size of nanoparticles produced by laser ablation of flowing aerosols," J. Nanoparticle Res. 2, 141-145(2000).

${ }^{91}$ Gokul Malyavanatham, Daniel T. O'Brien, Michael F. Becker, William T. Nichols, John W. Keto, Desiderio Kovar, Sebastien Euphrasie, Thomas Loue, and Philippe Pernod, "Thick films fabricated by laser ablation of PZT microparticles," submitted to J. of Mat. Res.
} 
Microluminescence spectra were obtained from areas $\sim 1 \mu \mathrm{m}$ in diameter of the NPs shown in Fig. 8.13. The excitation laser, $\lambda=$ $798 \mathrm{~nm}$, was focused onto the sample and spectra obtained through a microscope equipped with a holographic notch filter to block the laser light. The light was dispersed in a spectrometer and measured with an InAs CCD array detector. An example spectrum is shown in Fig. 8.14 which is similar to spectra obtained for ion implanted glasses. ${ }^{92}$ Similar spectra were obtained for samples at temperatures as low as $10 \mathrm{~K}$. Radiative lifetimes were only $0.5 \mathrm{~ms}$ shorter than that of the starting microparticles, indicating that the nanostructured materials did not significantly increase the defect or impurity quenching of the Er ions.

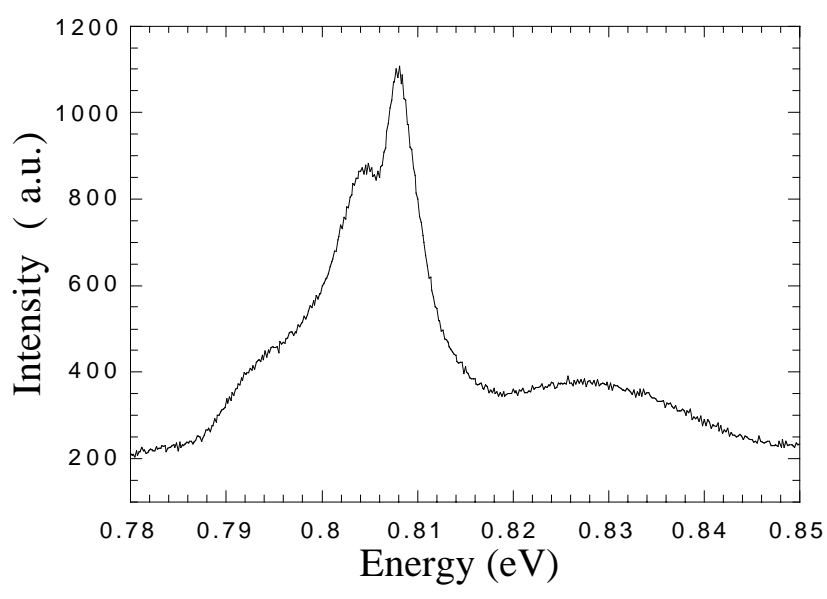

Fig. 8.14 Fluorescence of Er+ in glass NPs

More details of this research was recently submitted for publication in JOSA B. Applications of the nanoparticles include gain waveguides on integrated optical devices and nanoslush lasers.

92 A. Polman, D. C. Jacobson, D. J. Ealesham, R. C. Kisler, and J. M. Poate, J. Appl. Phys 70, 3778(1991). 


\section{Publications}

1. Jacek Borysow, Hossein Golnabi, Roger H. Taylor, and J. W. Keto, "Techniques for measuring small changes in the intensity of a pulsed laser", Applied Optics 31, 2814-2819(1992).

2. J. W. Keto, W. B. Layne, and M. R. Bruce, "Harpooning reactions of excited rare gase atoms", Gas-Phase Metal Reactions, A. Fontijn, ed., Elsevier (Amsterdam), p. 435-466(1992).

3. Joe P. Holder, P. Bhatia, and J. W. Keto, "Raman-Induced Kerr Effect Spectroscopy of Discharge Excited Molecules and Molecular Ions", Proceedings of the XIII International Conference on Raman Spectroscopy, Würzburg, Germany, 1992, e.d. W. Kiefer, et. al., John Wiley and Sons (West Sussex, England), p. 236, 1992.

4. C. A. Whitehead, H. Pournasr, and J. W. Keto, "Two-photon Absorption in Liquid Xenon", Phys. Rev. 47, 4979(1993).

5. R. Karnbach, M. C. Castex, J. W. Keto, M. Joppien, J. Wőrmer, G. Zimmerer, and T. Mőller, "Excited state dynamics of $\mathrm{Kr}_{\mathrm{N}}$ clusters probed with time and energy resolved photo luminescence methods", Chem. Phys. Letters. 203, 248(1993).

6. Parminder Bhatia, OHD-RIKE spectrometer using pulsed lasers: a step towards quantum limited detection, Ph. D. Thesis, The University of Texas at Austin, 1994.

7. Homayoun Pournasr-Khakbaz Two-photon absorption in condensed xenon, $\mathrm{Ph}$. D. Thesis, The University of Texas at Austin, 1994.

8. C. A. Whitehead, M. R. Bruce, Hong Cai, J. Kohel, W. B. Layne, and J. W. Keto,"Deactivation of Two-Photon Excited Xe(5p56p, 6p', 7p) and $\mathrm{Kr}(4 \mathrm{p} 55 \mathrm{p})$ in Xenon and Krypton", J. Chem. Phys.102,1965(1995)

9. H. Pournasr, Joe Holder and J. W. Keto, "Two-photon Spectroscopy of Excitons in Solid Xenon", Phys. Rev. B54, 864-870(1996).

10. Parminder S. Bhatia and J. W. Keto, "Narrow band, precisely tunable pulsed dye laser," Applied Optics 35, 4152-4159(1996).

11. Martin A. Kykta, The search for fluorescence from the excimer states correlating to $X d^{*}\left(5 p^{5} 6 p\right)$, Ph. D. Thesis, The University of Texas at Austin, 1996.

12. James M. Kohel, Two-photon laser-assisted reactive collisions in xenon and chlorine gas mixtures, Ph. D. Thesis, The University of Texas at Austin, 1996.

13. Hong Cai, Martin Kykta, Lei Chen, John W. Keto, G. Zimmerer, and T. Mőller, "Two-photon laser spectroscopy of xenon dimers and clusters in supersonic jets", J. Chem. Phys. 107, 6080(1997).

14. G. Guttroff, J. W. Keto, C. K. Shih, A. Anselm, and B. G. Streetman, Appl. Phys. Lett. 68, 3620 (1996).

15. Joe P. Holder, Two-collor studies of xenon $5 p^{5} 5 d$ and $5 p^{5} 6 d$ multiplets in rare gas buffers, $\mathrm{Ph}$. D. Thesis, The University of Texas at Austin, 1997. 
16. Parminder S. Bhatia, Joe P. Holder, and J. W. Keto, "Highly sensitive Rikes spectrometer using pulsed lasers", J. Opt. Soc. Am. B.14, 263-270(1997).

17. Parminder S. Bhatia, Craig McCluskey, and J. W. Keto, "Absolute calibration of a precision wavemeter for pulsed lasers", Appl. Optics 38, 1-13(1999).

18. Parminder S. Bhatia and J. W. Keto, "Pressure and power dependence of the OHD-RIKE line shape," Phys. Rev. A. 59, 4045-4051(1999).

19. H.Htoon, Hongbin Yu, D. Kulik, J. W. Keto, O. Baklenov, A.L.Holmes Jr., B.G. Streetman, and C.K.Shih, "Quantum dots at the nanometer scale: Interdot carrier shuffling and multiparticle states," Phys. Rev. B. 60, 11026-11029(1999).

20. H. Htoon, J. W. Keto, O. Baklenov, and A. L. Holmes, Jr., C. K. Shih, "Cross-sectional NanoPhotoluminescence Studies of Stark Effects in Self-Assembled Quantum Dots," Appl. Phys. Lett.76, 700-702(2000).

21. W.T. Nichols, G. Malyavanatham, M.P. Beam, D.E. Henneke, J.R. Brock, M.F. Becker, and J.W. Keto, "Synthesis of nanostructured wc films by supersonic impaction of nanoparticle aerosols," Nanophase and Nanocomposite Materials III, Vol. 581,(Material Research Soc., Pittsburg ), p. 193-198, 2000.

22. H. Htoon, Hongbin Yu, D. Kulik, J. Keto, C.K. Shih, O.l Baklenov, and A. L. Holmes, "Nanophotoluminescence studies of self-assembled quantum dots," Self-organized Processes in Semiconductor Alloys- Vol. 583 (Material Research Soc., Pittsburg), p. 105-111, 2000.

23. S. K. Jo, J. H. Kang, X. Yan, J. M. White, B. Gong, J. G. Ekerdt, J. Keto, and J. Lee, "Direct absorption of gas-phase atomic hydrogen by $\mathrm{Si}(100)$ : a narrow temperature window," Phys. Rev. Lett. 85, 2144-2147(2000).

24. William T. Nichols, Gokul Malyavanatham, Dale E. Henneke ,James R. Brock, Michael F. Becker, John W. Keto, and Howard D. Glicksman, "Gas and Pressure Dependence for the Mean Size of Nanoparticles Produced by Laser Ablation of Microparticles in Aerosols", J. of Nanoparticle Res. 2, 141-145(2000).

25. Ron Potok, "Design and Implementation of a Narrowband Ti:Sapphire Laser Amplifier," Senior Thesis, May, 2000.

26. W.T. Nichols, D.E. Henneke, G. Malyavanatham, M.F. Becker†, J.R. Brock, and J.W. Keto, and H. D. Glicksman, "Large scale production of nanocrystals by laser ablation of aerosols of microparticles," Appl. Phys. Lett. 78, 1128-1130(2001).

27. James M. Kohel and J. W. Keto, "Two-photon laser-assisted reactions in Xe and $\mathrm{Cl}_{2}$ gas mixtures," J. Chem. Phys. 113, 10551-10559(2001).

28. Jaemyoung Lee, Michael F. Becker, and John W. Keto, "Dynamics of laser ablation of microparticles prior to nanoparticle generation," J. of Applied Physics 89, 8146-8152(2001).

29. Adrianna G. Petrova, "Argon clusters with xenon impurities," Masters Thesis, Physics, The University of Texas at Austing, August 2001.

30. W. T. Nichols, G. Malyavanatham, D. T. O'Brien, D. Kovar, M. F. Becker, and J. W. Keto, "Supersonic nanocrystal deposition for nanostructured materials," Mat. Res. Soc. Symp. Proc. 703, (Material Research Soc., Pittsburg, 2002), p 5.51-5.56. 
31. W. T. Nichols, G. Malyavanatham, D. E. Henneke, J. R. Brock, M. F. Becker, and J. W. Keto, "Bimodal nanoparticle size distributions produced by laser ablation of microparticles in aerosols", J. of Nanoparticle Res. 4, 423-432(2002).

32. Craig W. McCluskey, The Search for Metastables and Molecular Ions in Discharges, Ph. D. Thesis, The University of Texas at Austin, June 2002.

33. J. W. Keto, W. Nichols, D. O'Brian, M. F. Becker, and D. Kovar, "Nanoparticles: Building Blocks for Nanostructured Materials," From the Atomic to the Nano-scale, Proceedings of the International Workshop, C. T. Whelan and J. H. McGuire, ed.,(Old Dominion, 2003) p. 54-62.

34. Dale E. Henneke, Gokul Malyavanatham, Desiderio Kovar, D. T. O'Brien, M. F. Becker and William T. Nichols, and J. W. Keto, "Stabilization of silver nanoparticles in nonanoic acid: a temperature activated conformation reaction observed with surface enhanced Raman spectroscopy," J. Chem. Phys. 119, 6802-6809(2003).

35. Sandra Marianne Lang, "Mass Spectroscopy of Cold Silicon and Silver Clusters," Masters Thesis, Physics, The University of Texas at Austing, August 2004.

36. Michael Gerbracht, "A near-field scanning optical microscope" Masters Thesis, Physics, The University of Texas at Austing, August 2004.

37. Mohammed M. Sahrapour, "NSOM: Low temperautre near-field scanning optical microscope" Seniour Thesis, Physics, The University of Texas at Austing, May 2004.

38. D. Stoker, M. F. Becker, and J. W. Keto, "Optical third-harmonic microscopy using ultrashort laser pulses,” submitted Rapid Comm. to Phys. Rev.

39. John W. Keto, Gokul Malyavanathm, Andreas Muller, Daniel T. O’Brien, C. K. Shih, Michael. F. Becker, and Desiderio Kovar, "Nanoparticles of Er doped glass produced by laser ablation of microparticles,” submitted JOSA B.

40. D. Stoker, Jonghoon Baek, M. F. Becker, Desiderio Kovar, Weijie Wang, and J. W. Keto, "Optical third-harmonic generation in $\mathrm{Al}_{2} \mathrm{~N}_{3}$ thin films" submitted Phys. Rev. A.

41. William T. Nichols, Gokul Malyavanatham, Michael F. Becker, and John W. Keto, "Fluoescence of CdSe and Si nanocrystals produced by the laser ablation of microparticles," in preparation. 
1. Invited: Joe P. Holder, P. Bhatia, and J. W. Keto, "Raman-Induced Kerr Effect Spectroscopy of Discharge Excited Molecules and Molecular Ions", XIII International Conference on Raman Spectroscopy, Würzburg, Germany, 1992.

2. C.A.Whitehead, H.Pournasr, and J.W.Keto, "Two-Photon Absorption In Liquid Xenon", Meeting of DAMOP, Bull. Am. Phys. Soc. 37, 1992.

3. H. Pournasr, C.A. Whitehead, M.R. Bruce, Hong Cai, J. Kohel, W.B. Layne, and J.W. Keto, "Deactivation of Two-Photon Excited Xe(5p56p,6p',7p) and $\mathrm{Kr}(4 \mathrm{p} 55 \mathrm{p})$ in Xenon and Krypton.", Meeting of DAMOP, Bull. Am. Phys. Soc. 37, 1992.

4. J. W. Keto "Excitation transfer to krypton in collisions with state-selectively excited xenon atoms", XIII U.S. DOE Atomic Physics Program Workshop, Kansas State University, Oct. 1992.

5. Invited: J. W. Keto, "Harpooning Reactions of Excited Rare Gas Atoms", Atomic Physics Seminar, Texas A\&M University, March 1993.

6. Invited: J. W. Keto, "Rare Gas Dimers, Clusters, and Other Bits", Physics Colloqium, Michigan Technological University, Houghton, Michigan, Oct. 1, 1993

7. J. W. Keto, "State-to-state energy transfer in atomic collisions", XV U.S. DOE Atomic Physics Program Workshop, University of Kentucky, Lexington, Kentucky, Oct. 14-15 1994.

8. J. W. Keto, "Crystal balls and golden cubes", Physics Seminar, University of Texas at Austin, September 21, 1994

9. J. Kohel and J. W. Keto, "Vibrational Energy Disposal in Two-Photon Laser-Assisted Reactions of Xe $+\mathrm{Cl}_{2}$ Collision Pairs," 26th Annual Meeting of the Division of the Atomic, Molecular and Optical Physics of the American Physical Society, May 16-19, 1995, Bull. Am. Phys. Soc. 40.4, 1346 (1995).

10. J. Kohel and J. W. Keto, "Two-photon laser-assisted reactive collisions in $\mathrm{Xe} / \mathrm{Cl}_{2}$ gas mixtures," Joint APS and AAPT and SPS Zone 13 Meeting, Oct. 26-28, 1995

11. Jaemyoung Lee, Michael F. Becker, James R. Brock, John W. Keto, and Rodger M. Walser, "Permalloy nanoparticles generated by laser ablation," International Magnetics Conference, Honolulu, Hawaii, Jan. 1996.

12. Invited: John W. Keto, "Generation of Metal Nanoparticles by Laser Ablation of Microspheres," NSF Joint U. S. Japan Workshop on Nanoparticle Synthesis and Applications, Oct 28, 1996, Tsukuba, Japan.

13. Invited: John W. Keto, "Crystal Balls and Golden Cubes," University of Texas Meeting for Secondary Teachers, April 12, 1997.

14. Invited: Michael F. Becker James R. Brock, Hong Cai, Nirmal Chaudhary, Dale Henneke, Laurent Hilsz, John W. Keto, Jaemyoung Lee, Will Nichols, and Howard D. Glicksman, 
"Nanoparticles generated by laser ablation," Joint NSF-NIST Conference on Ultrafine Particle Engineering, NSF (Arlington, VA), May 12, 1997.

15. Michael F. Becker, James R. Brock, Hong Cai, Dale Henneke, Laurent Hilsz, John W. Keto, Jaemyoung Lee, William T. Nichols, and Howard D. Glicksman, "Nanoparticles generated by laser ablation," 1998 Conf. Lasers and Electro-Opt. and Intn'l Quant. Elect. Conf., San Francisco, CA, May 6, 1998.

16. Michael F. Becker, James R. Brock, Hong Cai, Dale Henneke, John W. Keto, Jaemyoung Lee, William T. Nichols, and Howard D. Glicksman, "Metal nanoparticles generated by laser ablation," 17th Annual Meeting for Am. Assoc. for Aerosol Research, Cincinnatti, Ohio, June 22-26, 1998.

17. Invited: "Crystal Balls and Golden Cubes," Texas Section of the APS/AAPT, University of North Texas, Denton, TX, October 10-11, 1997.

18. Invited: "Nanoparticles Generated by Laser Ablation of Microspheres," Center of Excellence Distinguished Scientist Lecture, National Institute of Materials and Chemistry, Agency of Industrial Science and Technology, Tsukuba, Japan, Feb. 3, 1998.

19. Invited: Michael F. Becker, James R. Brock, Hong Cai, John W. Keto, Jaemyoung Lee and William T. Nichols, "Metal Nanoparticles generated by laser ablation," 1998 TMS (Minerals Metals and Materials) Annual Meeting, Feb. 16, 1998.

20. John W. Keto, Coherent Raman Spectroscopy of Cluster ions and Radicals in Discharges, AMO Research Mtg., U.S. Dept. of Energy, BES, Oct. 1998.

21. William T. Nichols, Dale Henneke, Gokul Malyavanatham, Michael F. Becker, James R. Brock, John W. Keto, "High volume production of metallic nanoparticles," Centenial Meeting of the Am. Phys. Soc., Vol. 44, p. 1693, Atlanta, GA, March 20-26.

22. Invited: William T. Nichols, Dale Henneke, Gokul Malyavanatham, Jaemyoung Lee, Michael F. Becker, James R. Brock, John W. Keto, Howard D. Glicksman, "High volume production of silver nanoparticles," First Joint ESF-NSF Symposium on Aerosols for Nanostructured Materials and Devices, Edinburgh, Scotland, Sept. 1998.

23. W.T. Nichols, G. Malyavanatham, D.E. Henneke, J.R. Brock, M.F. Becker and J.W. KetoAAAR, Tacoma, WA. Oct. 11-15, 1999. "Laser Ablation of Aerosols for the Production of Nanoparticles".

24. G.Malyavanatham, D.T.O'Brien, S.Euphrasie, W.T.Nichols, D.E.Henneke, D.Kovar, J.W.Keto, and M.F.Becker, TEXMEMS II, Dallas, TX, 16 May 2000. "Supersonic Deposition of PZT Nanoparticles for Functional Applications."

25. John W. Keto, "Spectroscopy of Cluster Ions in Discharges, Clusters in Jets, and Nanoparticles," AMO Research Program: Molecular Ions, Nanostructures and Surfaces, U.S. DoE, Oct. 27, 1999.

26. Invited: John W. Keto, "Spectroscopy of Nanoparticles", Physics Colloquim, University of Louisville, Feb. 2000.

27. W.T. Nichols, G. Malyavanatham, D.T. O’Brien, W. Wang, D. Kovar, M.F. Becker, J.W. Keto, and H.D. Glicksman, "Nano-aerosol processing: from nanocrystallites to nanostructured thick films and composites," Abstracts of Am. Assoc. of Aerosol Res., St. Louis, MO, Nov. 4, 2000 . 
28. G. Malyavanatham*, D.T. O’Brien, W.T. Nichols, J.W. Keto, D. Kovar and M.F. Becker; H.D. Glicksman, DuPont Corporation low sintering temperature nanostructured materials prepared by laser ablation of aerosols," Abstracts of Am. Assoc. of Aerosol Res., St. Louis, MO, Nov. 4, 2000.

29. D. T. O’Brien, G. Malyavanatham, S. Euphrasie, T. Loué, W. T. Nichols, M. F. Becker; D. Kovar, J. W. Keto, P. Pernod*, "PZT films fabricated by the jet deposition of nanoparticles," TexMEMS Conference Abstracts, Southern Methodist University, Dallas TX, 5 June 2001.

30. Invited: John W. Keto, "Nanoparticles: Building blocks of nanostructures,"IEMN, Ecole Central de Lille, Lille, France, June 25, 2001.

31. Invited: John W. Keto, "Jet deposition of fine metal lines using metal nanoparticles," Dupont Electronics Corp., Rayleigh, NC.,July 7, 2001

32. D. T. O'Brien, G. Malyavanatham, W. T. Nichols, J. W. Keto, D. Kovar, M. F. Becker, and H.D. Glicksman, "Low sintering temperature nanostructured materials prepared by laser ablation of aerosols," 20th Annual AAAR Conference, Oct. 2001, Portland OR.

33. W.T. Nichols, G. Malyavanatham, D. T. O'Brien, W. Wang, J. W. Keto, D. Kovar, M. F. Becker, J. W. Keto, and H.D. Glicksman, "Nano-aerosol processing: from nanocrystallites to nanostructured thick films and composites," Presented Oct. 2001, 20th Annual AAAR Conference, Portland OR.

34. Invited: W.T. Nichols, D.T. O'Brien, G. Malyavanatham, M.F. Becker, and J.W. Keto, "Control of Nanoaerosols for Production of Nanostructured Materials," Presented 27 June 2002 International Conference on Nanometer-scale Science and Technology VII (NANO7) Malmo, Sweden.

35. Invited: John W. Keto, "Manufacture and Application of Nanoparticles." Presented at the NSF Congressional symposium, "Small Wonders: Exploring the Vast Potential of Nanoscience." March 18-19, 2002.

36. Invited: John W. Keto, "AMO Physics in Nanotechnology," Review lecture, TAMOC Meeting of the Div. of AMO physics, Am. Phys. Soc., Williamsburg, VA, March 28, 2002

37. Invited: W.T. Nichols, G. Malyavanatham, D.T. O,Brien, D. Kovar, M.F. Becker, and J.W. Keto. "A Journey in Nanomanufacturing: from hot atoms to cool materials" Presented 17 June 2002, United Engineering Foundation Conference on Nanoparticles and Nanostructures through Vapor Phase Synthesis IV, Barga, Italy.

38. M. F. Becker, J. R. Brock, J. W. Keto, D. Kovar, W. T. Nichols, S. Euphrase, G. Malyavanatham, D. T. O'Brien, "Nanostructured Thick Films Produced by Impaction," Symposium on Processing, Characterization and Properties of Nanostructured Materials , 54th Pacific Coast Meeting of the American Ceramic Society, Friday, October 4, 2002

39. G. Malyavanatham, D.T. O’Brien, M.F. Becker D. Kovar and J.W. Keto, characterization study of Au-Cu binary alloy nanoparticles, Am. Assoc. of Aerosol Res., Oct. 7-9, 2002.

40. Invited: M. F. Becker, J. R. Brock, J. W. Keto, D. Kovar, W. T. Nichols, D. E. Henneke, G. Malyavanatham, D. T. O'Brien "Review of laser ablation of microparticles," Am. Assoc. of Aerosol Res., Oct. 7-9, 2002. 
41. G. Malyavanatham, D.T. O’Brien, M.F. Becker, J.W. Keto, and D. Kovar, Au-Cu binary alloy nanoparticles, Mat. Res. Soc., Dec. 5-9, 2002, Boston, MA.

42. D. T. O’Brien, N. Descaszeaud, J. W. Keto, D. Kovar, and M. F. Becker, "Low temperature sintering of nanostructured silver prepared by laser ablation of aerosols," SPRING Consortium Conf. , Univ. of TX at Austin, August 26, 2003.

43. M. F. Becker, J. W. Keto, D. Kovar, W. T. Nichols, D. E. Henneke, G. Malyavanatham, D. T. O'Brien, W. Wang, J. Baek, J. Ma, C. Wong, C. Lai, D. Stoker, "Review of laser abation of microparticles," SPRING Consortium Conf., Univ. of TX at Austin, August 26, 2003.

44. Invited: John W. Keto, "Nanoparticles: building blocks for nanostructures," Invited paper at the conference, 'From the Atomic to the Nano Scale' Old Dominion University, Dec. 12-14, 2002.

45. Invited: John W. Keto, "Nanoparticles: building blocks for nanostructures," Texas A\&M University, Feb. 18, 2003.

46: Invited: John W. Keto, "Manufacture of Nanoparticles and Nanocomposites Using Laser Ablation," The Texas Alliance for Technology Commercialization, June 13, 2003.

47. Gokul Malyavanatham, Desiderio Kovar, Daniel T. O'Brien, Michael F. Becker, William T. Nichols, and John W. Keto, "Alloy Nanoparticles Produced from Laser Ablation of Elemental Microparticle Aerosols," 22th Annual AAAR Conference, Annaheim CA, October 20-24, 2003.

48. Weijie Wang, Daniel T. O’Brien, Jonghoon Baek, Michael F. Becker, John W. Keto, Desiderio Kovar , "Nanocomposites Prepared by Laser Ablation of Microparticles (LAM) and Subsequent Thin Film Deposition,” Mat. Res. Soc., Dec. 1-5, 2003, Boston, MA.

49. James T. Ma, Daniel T. O’Brien, Michael F. Becker, John W. Keto, and Desiderio Kovar "Nanoscale Manufacturing - Nanocomposites for Magnetostrictive Actuators" NSF Nanoscale Science and Engineering Grantees Conference, Arlington VA, December 16-18, 2003.

50. Invited: Michael F. Becker, John W. Keto, and Desiderio Kovar, "Nanoscale Manufacturing Nanocomposites for Magnetostrictive Actuators," 2004 National Science Foundation Design, Service and Manufacture and Industrial Innovation Grantees and Research Conference, Dallas, Texas, January 5-8, 2004.

51. Invited: D. Kovar, M.F. Becker, J.W. Keto, D.T. O’Brien, J. Baek, J. Ma, A. Albert, C. Huang, W. Wang, C. Li, and D. Stoker, "Nanoparticles and Nanostructured Films Produced by Laser Ablation of Microparticles," XIII International Materials Research Congress, Cancun, Mexico, August 26, 2004.

52. Invited: D. Kovar, M.F. Becker, J.W. Keto, D.T. O’Brien, J. Baek, J. Ma, A. Albert, C. Huang, W. Wang, C. Li, and D. Stoker, "Direct Writing of Patterned Films Using Nanoparticles Produced by LAM," DARPA Workshop on Bridging Direct Write Technology Dimensions, Seward AK, June 27-July 1, 2004.

53. D. Stoker, J. Baek, W. Wang, M. F. Becker, Desi Kovar, John W. Keto, "Building Nanostructured Materials for Nonlinear Optics," Nano-Night, UT's university-wide Poster Session on nanoscience and nanotechnology, Austin TX, April 23, 2004.

54. Weijie Wang, Jonghoon Baek, Michael F. Becker, Chong Huang, Daniel T. O'Brien, David Stoker, John W. Keto, and Desiderio Kovar, "Nanocomposites Prepared by Laser Ablation of 
Microparticles (LAM) and Subsequent Thin Film Deposition," Nano-Night, UT's university-wide Poster Session on nanoscience and nanotechnology, Austin TX, April 23, 2004.

55. Invited: John W. Keto, "Nanoparticles: building blocks for photonic nanostructures," NSF Focus conference, 'From the Atomic to the Nano Scale' The University of Texas at Austin, Feb. 67, 2004

56. Changyi Lai, Chong Huang, Desiderio Kovar, John W. Keto, and Michael F. Becker, "Laser Assisted Nanoparticle

57. J. W. Keto, Focusing in Nanoscale Direct Writing," Nanoscale Dev. and Syst. Integration, Houston, TX, April 4-6,05.

58. Ignacio F. Gallardo, Kay Hoffmann, John W. Keto, "Characterization of Semiconductor CoreShell Nanoparticles Generated by Laser Ablation of Microparticles," Nano-Night, April 22, 05

59. D. Stoker, Jonghoon Back, Desidero Kovar, M. F. Becker, and John W. Keto, "Opt. thirdharmonic microscopy of nano-structured thin films using ultra-short pulsed lasers," Nano-Night, Aprill 22, 05

60. D. Stoker, M. F. Becker, M. C. Downer, and J. W. Keto, "Opt. third-harmonic surface microscopy using ultra-short pulsed lasers,” OSI VI, Coppenhangen, Denmark, June 05.

61. D. Stoker, J. Back, M. C. Downer, D. Kovar, M. F. Becker, and J. W. Keto, "Interferometric tird-harmonic generation in thin optical films using ultra-short laser pulses," Frontiers in Optics," Laser Science XXI, Tucson, AZ, Oct. 16-20, 2005.

62. D. Stoker, J. Back, M. C. Downer, D. Kovar, M. F. Becker, and J. W. Keto, Viscous sintering of aerosol-written nanostructured glass films, AAAR, Austin, TX, October 10-15(2005)

63. Invited: Desidero Kovar, M. F. Becker, and J. W. Keto, "Scalable methods for producing three dimensional nanocomposites for energy, semiconductor, and advanced manufacturing applications," Ready to Commercialize 2005, J. J. Pickle Center, The University of Texas at Austin, May 24, 2005. 


\section{Personnel}

The following students contributed to DOE research over the past 10 years: Hong Cai, Martin Kykta, Craig McClusky, Homahoung Pournasr, Joe Holder, David Stoker have been supported by DOE. The remaining students have been supported by the Robert A. Welch Foundation, the University of Texas Physics Department, or for the Wurtzburg exchange student, by the University of Wurtzburg.

1. Hong Cai

2. Parmenter Bhatia

3. Xiaoqun Duan

4. Ingolf Hamp

5. Jeremy Highly

6. James Kohel

7. Martin Kykta

8. Sandra Lang

9. Barathi Meduri

10. Thomas Neuberger

11. Andriana Petrova

12. Craig McClusky

13. Homayoun Pournasr

14. Georg Ernst Sternecker

15. David Stoker

16. Joe Ting
PhD August 1995, "Experiments on rare gas clusters and nanoparticles"

PhD June 1995

Undergraduate research assistant

MS December 1994, Exchange student of University of Wurtzburg

Undergraduate research assistant

PhD 1995 "Two photon laser assisted reactive collisons in xenon and chlorine gas mixtures"

PhD January 1996, "Search for fluorescence from xenon excimers correlating to $\mathrm{Xe}^{*} 5 \mathrm{p}^{5} 6 \mathrm{p} "$

MS 2004 Mass spectroscopy of cold Si and Ag clusters

MS December 1995, "Design and construction of a multipass Tisapphire amplifier"

MS December 1994, Exchange student of University of Wurtzburg

MS 2001

PhD 2002 "The search for metastables and molecular ions in discharges"

PhD June 1995, "Two-photon absorption in condensed xenon"

MS August 1994, Exchange student of University of Wurtzburg

PhD candidate

Undergraduate research assistant 